\title{
Crystalline Phase Separation in Phosphate Containing Waste Glasses: Relevance to INEEL HAW
}

by

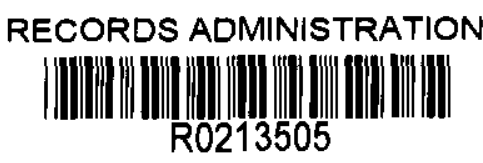

C. M. Jantzen

Westinghouse Savannah River Company

Savannah River Site

Aiken, South Carolina 29808

K, G. Brown

J. B. Pickett

G. L. Ritzhaupt

Oral Roberts University

OK USA

DOE Contract No. DE-AC09-96SR18500

This paper was prepared in connection with work done under the above contract number with the U. S.

Department of Energy. By acceptance of this paper, the publisher and/or recipient acknowledges the U. S.

Government's right to retain a nonexclusive, royalty-free license in and to any copyright covering this paper, along with the right to reproduce and to authorize others to reproduce all or part of the copyrighted paper. 


\section{Crystalline Phase Separation in Phosphate Containing Waste Glasses: Relevancy to Vitrification of Idaho National Engineering and Environmental Laboratory (I NEEL) High Activity Waste (U)}

C. M. Jantzen, K.G. Brown, J.B. Pickett, and G.L. Ritzhaupt

Prepared for.

Tank Waste Focus Area

TTP\# SR1-6-WT-31

Subtask B

Milestone B.2-4

Approved by

E.W. Holtzscheiter, Manager

Immobilization Technology Section

Publication Date: September 30, 2000

Westinghouse Savannah River Company

Savannah River Site

Savannah River Technolagy Center

Aiken, SC 29808

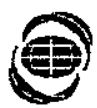

We Put Science to Work

PREPARED FOR THE U.S. DEPARTMENT OF ENERGY UNDER CONTRACT NO. DE-AC09-96SRI8500 


\section{DISCLAIMER}

This report was prepared as an account of work sponsored by an agency of the United States Government. Neither the United States Government nor any agency thereof, nor any of their employees, makes any warranty, express or implied, or assumes any legal liability or responsibility for the accuracy, completeness, or usefulness of any information, apparatus, product or process disclosed, or represents that its use would not infringe privately owned rights. Reference herein to any specific commercial product, process or service by trade name, trademark, manufacturer, or otherwise does not necessarily constitute or imply its endorsement, recommendation, or favoring by the United States Government or any agency thereof. The views and opinions of authors expressed herein do not necessarily state or reflect those of the United States Government or any agency thereof.

This report has been reproduced directly from the best available copy.

Available for sale to the public, in paper, from: U.S. Department of Commerce, National Technical Information Service, 5285 Port Royal Road, Springfield, VA 22161, phone: (800) 553-6847

fax: (703) 605-6900

email: orders@ntis.fedworld.gov

online ordering: http://www.ntis.gov/ordering.htm

Available electronically at http://www.doe.gov/bridge

Available for a processing fee to U.S. Department of Energy and its contractors, in paper, from: U.S. Department of Energy, Ofrice of Scientific and Technical Information, P.O. Box 62, Oak Ridge, TN 37831-0062, phone: (865) 576-8401

fax: (865) 576-5728

email: reports@adonis.osti.gov 
WSRC-TR-2000-00339

WSRC-TR-2000-00339

Distribution Category: Unlimited Keywords: Glass, High Level Waste, phase separation

Retention: Permanent

Crystalline Phase Separation in Phosphate Containing Waste Glasses: Relevancy to Vitrification of Idaho National Engineering and Environmental Laboratory (INEEL) High Activity Waste (U)

C. M. Jantzen and K.G. Brown Savannah Riwer Technology Center Aiken, SC 29803

and

J.B. Pickett

Westinghouse Savannah River Company Aiken, SC 29803

and

G.L. Ritzhaupt

Department of Chemistry

Oral Roberts University

Tulsa, OK 74171

Publication Date: September 30, 2000 


\section{Approvals}

$\frac{\text { C.m- } \operatorname{manh} \text { tien }}{\text { C. M. Jantzenf Authop Immobilization Technology }}-\frac{9 / 11 / 2000}{\text { Date }}$

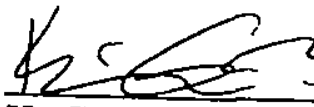

K. G. Brown, Author, Immobilization Technology

$\frac{9 / 11 / 2008}{\text { Date }}$

Dises for

D. K. P(eeler, Techpical Reviewer, Immobilization Technology $\frac{9-12-\infty}{\text { Date }}$

torifer

J. D. (Vienna, Tethnical Reviewer, Pacific Northwest Natl. Lab $\frac{9-12-\infty 8}{\text { Date }}$

Deris for

D. A Crowley, Ifevel 4 Manager, Immobilization Technology $\frac{9-12-00}{\text { Date }}$

Reriar

E.W. Holtzscheiter, Level 3 Manager, Immobilization Technology Date $\frac{9-12-0}{0}$ 


\section{EXECUTIVE SUMMARY}

Phase separation is typically defined as the separation, upon cooling, of a homogeneous glass melt into two or more phases. When the two phases are both amorphous (e.g., amorphous phase separation (APS) or glass-in-glass phase separation) glass durability is governed by durability of the continuous phase. The impacts of APS on complex borosilicate waste glasses has been shown to be detrimental to glass durability although the exact type and extent of phase separation is not well know. When the two separating phases are amorphous at the melt temperature but crystallize upon cooling the phenomena is called crystalline phase separation (CPS). ${ }^{\dagger}$ Less is known about the impact of CPS on borosilicate waste glass durability than about the impact of APS on borosilicate waste glass durability. Since the presence of APS is known to limit HLW waste loadings, it is important to understand the impact of CPS.

One option for immobilization of high level waste at the Idaho National Engineering and Environmental Laboratory (INEEL) is to dissolve calcined waste and then separate the High Activity Waste (HA.W) portion for vitrification. One of the proposed separation processes concentrates the radionuclides in the HAW but adds large concentrations of $\mathrm{P}_{2} \mathrm{O}_{5}$. Concentrations of $\mathrm{P}_{2} \mathrm{O}_{5}$ in excess of 2.5-3 wt\% are known to cause crystalline phase separation (CPS) in borosilicate waste glasses. The phase separated phosphate rich regions are discreet liquid droplets at the melt temperature that transform into crystalline alkali phosphate phases upon cooling under even the most rapid cooling conditions.

The $\mathrm{P}_{2} \mathrm{O}_{5}$ containing borosilicate glass compositions examined in this study contained between $0.06-13.5 \mathrm{wt} \% \mathrm{P}_{2} \mathrm{O}_{5}$. Some glasses had undergone CPS while other glasses had undergone amorphous phase separation (APS). Many of the higher $\mathrm{P}_{2} \mathrm{O}_{5}$ containing glasses that underwent CPS were more durable than the glasses containing lower $\mathrm{P}_{2} \mathrm{O}_{5}(<2.6 \mathrm{wt} \%)$ that underwent APS. Mathematical analysis of the glass durability data showed that high $\mathrm{Al}_{2} \mathrm{O}_{3}$ and $\mathrm{ZrO}_{2}$ concentrations in the glass had the following effects:

- improved the durability of the homogeneous glasses

- stabilized high $\mathrm{B}_{2} \mathrm{O}_{3}$ containing glasses from undergoing APS

- improved the durability of the glassy matrix in glasses undergoing CPS

$\dagger$ As used in this study, CPS refers to glasses that are immiscible (exhibit droplet formation of a second liquid phase) at the melt temperature: the droplets transform during cooling into crystalline phases, e.g. the matrix phase remains amorphous while the droplets crystallize. 
The high $\mathrm{Al}_{2} \mathrm{O}_{3}$ content did not stabilize the glass against CPS as it does in borosilicate glasses that undergo APS. Alumina and zirconia were found to stabilize the CPS glass matrix against APS and stabilize the homogeneous glasses against APS. All INEEL glasses with a combined concentration of $\mathrm{Al}_{2} \mathrm{O}_{3}$ and $\mathrm{ZrO}_{2} \geq 9 \mathrm{wt} \%$ were found to have adequate durability

Due to the crystalline nature of the $\mathrm{P}_{2} \mathrm{O}_{5}$ phase separation and the minimal effect of the CPS phases on durability (based on the normalized release of $\mathrm{B}$ and $\mathrm{Na}$ ), CPS should be treated as a crystallization phenomena rather than a phase separation phenomena for waste acceptance. It remains to be demonstrated that the radionuclides do not preferentially partition into the CPS phase and leach at a greater rate than the $\mathrm{B}$ or $\mathrm{Na}$ although there was no evidence in this study that uranium partitioned into the CPS phases. It also must be shown that the formation of the phosphate rich CPS phase at the melt temperature where it is still in liquid form does not adversely effect the processability of the glass, i.e. electrical resistivity and/or incompatibilities with materials of construction.

It is recommended that glass composition regions for future study include those in the table below if the high $\mathrm{P}_{2} \mathrm{O}_{5}$ separations process is chosen for INEEL.

\begin{tabular}{|l|l|}
\hline $\begin{array}{l}\text { Glass Compositional } \\
\text { Component(s) }\end{array}$ & Ranges $(1 \mathrm{~T} \%)$ \\
\hline $\mathrm{B}_{2} \mathrm{O}_{3}$ & $\geq 2 \mathrm{wt} \%$ and $\leq 14 \mathrm{wt} \%$ \\
\hline $\begin{array}{l}\mathrm{Na}_{2} \mathrm{O}+\mathrm{Li}_{2} \mathrm{O}+\mathrm{Cs}_{2} \mathrm{O}+ \\
\mathrm{K}_{2} \mathrm{O}\end{array}$ & $\leq 22 \mathrm{wt} \%$ \\
\hline $\mathrm{P}_{2} \mathrm{O}_{5}$ & 0.06 to $>13.25$ \\
\hline $\mathrm{Al}_{2} \mathrm{O}_{3}$ & $0-25$ \\
\hline $\mathrm{ZrO}_{2}$ & $0-15$ \\
\hline $\mathrm{Al}_{2} \mathrm{O}_{3}+\mathrm{ZrO}_{2}$ & $\geq 9$ \\
\hline
\end{tabular}


WSRC-TR-2000-00339

This page intentionally left blank.

$\bullet$ 


\section{TABLE OF CONTENTS}

1.0 INTRODUCTION

$2.0 \quad$ AMORPHOUS VS. CRYSTALLINE PHASE SEPARATION ................... 2

3.0 HISTORICAL BACKGROUND ......................................................... 7

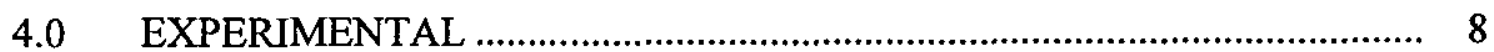

5.0 TYPES OF CRYSTALLINE PHASE SEPARATION (CPS) ........................ 9

5.1 CPS in Mixed Waste Glasses: Pilot Scale Testing .................................... 20

5.2 CPS in Borosilicate Glasses: Crucible and Pilot Scale Testing ................ 23

6.0 EFFECTS OF CRYSTALLINE PHASE SEPARATION ON GLASS DURABILITY.

7.0 PREDICTING GLASS DURABILITY FOR HIGH $\mathrm{P}_{2} \mathrm{O}_{5}$ CONTAINING GLASSES WITH CPS

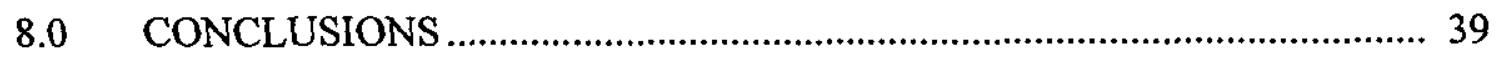

9.0 ACKNOWLEDGEMENTS ................................................................. 39

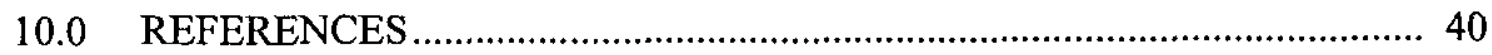




\section{WSRC-TR-2000-00339}

\section{LIST OF ILLUSTRATIONS}

1 Example of Type A phase separation in sodium borosilicate glass .............. 4

2 Example of Type B phase separation in sodium borosilicate glass............... 4

3 Example of Type $\mathrm{C}$ phase separation in sodium borosilicate glass................ 5

4 CaO- $\mathrm{P}_{2} \mathrm{O}_{5}$ rich droplets which have crystallized in a phosphate rich borosilicate glass

$5 \quad \mathrm{Li}_{3} \mathrm{PO}_{4}$ crystalline droplets in an SRS phosphate rich borosilicate glass. ....... 6

6a Phosphate glass crystalline phase separation (CPS) began in glasses produced during pilot scale melter runs

6b Alumina contents as high as $15 \mathrm{wt} \%$ did not stabilize the glass against CPS.

7a Data from 91 borosilicate waste glasses containing varying amounts of $\mathrm{P}_{2} \mathrm{O}_{5}$ plotted against $\mathrm{Al}_{2} \mathrm{O}_{3}$

7b Data from 91 borosilicate waste glasses containing varying amounts of $\mathrm{P}_{2} \mathrm{O}_{5}$ plotted against $\mathrm{B}_{2} \mathrm{O}_{3}$

8 Plot of $\log$ NL(B) vs. $\log$ NL(Na) for 82 amorphous and CPS glasses 26

9a Release of normalized $\mathrm{B}$ vs. Li for 50 homogeneous $\mathrm{P}_{2} \mathrm{O}_{5}$ glasses 27

9b Release of normalized B vs. Li for 37 crystallized CPS glasses ................... 28

10 Release of normalized Li vs. P for 24 crystallized INEEL CPS glasses......... 29

$11 \mathrm{a}$ The durability of the $\mathrm{P}_{2} \mathrm{O}_{5}$ containing borosilicate glasses as a function of the $\mathrm{P}_{2} \mathrm{O}_{5} /\left(\mathrm{SiO}_{2}+\mathrm{B}_{2} \mathrm{O}_{3}\right)$ molar ratio.

$11 \mathrm{~b}$ Borosilicate glasses with $>14 \mathrm{wt} \% \mathrm{~B}_{2} \mathrm{O}_{3}$

12 Glass durability is a function of the strong base and weak acid equilibria in the leachate and not a function of CPS 
13a Durable glass population when Ealkali oxides is limited to $19.3 \mathrm{wt} \%$........... 34

13b Durable glass population when Ealkali oxides is limited to $22 \mathrm{wt} \%$.............. 35

14a Contour plot of the PCT leachate response ……………................................ 37

14b. Individual PCT leachate responses.............................................................. 38 


\section{WSRC-TR-2000-00339}

\section{LIST OF TABLES}

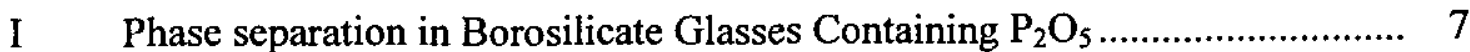

II Database of Borosilicate Glasses Containing $\mathrm{P}_{2} \mathrm{O}_{5}$ : Fabrication Methods and Homogeneity

III Database of Borosilicate Glasses Containing $\mathrm{P}_{2} \mathrm{O}_{5}$ : Compositions............... 13

IV Database of Borosilicate Glasses Containing $\mathrm{P}_{2} \mathrm{O}_{5}$ : Durability Response ...... 17

V Borosilicate Glasses Containing $\mathrm{Li}_{2} \mathrm{O}$ and Exhibiting CPS ....................... 20

VI Compositional Region Defined for Durable High $\mathrm{P}_{2} \mathrm{O}_{5}$ Containing Borosilicate Glasses Including Homogeneous and CPS Glasses 


\section{LIST OF ACRONYMS}

$\begin{array}{ll}\text { APS } & \text { Amorphous Phase Separation } \\ \text { ASTM: } & \text { American Standards and Testing Materials } \\ \text { CPS } & \text { Crystalline Phase Separation } \\ \text { DOE: } & \text { United States Department of Energy } \\ \text { DWPF: } & \text { Defense Waste Processing Facility } \\ \text { HAW: } & \text { High Activity Waste } \\ \text { INEEL: } & \text { Idaho National Engineering and Environmental Laboratory } \\ \text { PCT: } & \text { Product Consistency Test (ASTM C1285) } \\ \text { [SB] } & \text { Concentration of Strong Bases in Leachates } \\ \text { SEM: } & \text { Scanning Electron Microscopy } \\ \text { SRS: } & \text { Savannah River Site } \\ \text { SRTC: } & \text { Savannah River Technology Center } \\ \text { TEM: } & \text { Transmission Electron Microscopy } \\ \text { [WA] } & \text { Concentration of Weak Acids in Leachates } \\ \text { WSRC: } & \text { Westinghouse Savannah River Company } \\ \text { XRD: } & \text { X-Ray Diffraction }\end{array}$




\title{
Crystalline Phase Separation in Phosphate Containing Waste Glasses: Relevancy to Vitrification of Idaho National Engineering and Environmental Laboratory (INEEL) High Activity Waste (U)
}

\author{
C. M. Jantzen, K.G. Brown, and J.B. Pickett \\ Westinghouse Savannah River Company \\ Aiken, South Carolina 29808
}

and

\author{
G.L. Ritzhaupt \\ Department of Chemistry \\ Oral Roberts University \\ Tulsa, Oklahoma 74171
}

\subsection{INTRODUCTION}

As part of the Tanks Focus Area's (TFA) effort to increase waste loading for high-level waste vitrification at various facilities in the Department of Energy (DOE) complex, the occurrence of phase separation in waste glasses spanning the Savannah River Site (SRS) and Idaho National Engineering and Environmental Laboratory (INEEL) composition ranges have been studied. The type of phase separation that occurs in the phosphate rich borosilicate waste glasses, such as those investigated for INEEL, crystallizes upon cooling. This type of phase separation mechanism is less well studied than amorphous phase separation in phosphate poor borosilicate waste glasses. Therefore, the type of phase separation, extent, and impact of phase separation on glass durability for a series of INEEL-type glasses were examined and the data statistically analyzed in this study.

Phase separation is typically defined as the separation, upon cooling, of a homogeneous glass melt into two or more phases. When the two phases are both amorphous (e.g. amorphous phase separation (APS) or glass-in-glass phase separation) glass durability can be governed by the least durable continuous phase (Types A and B) or by the most durable continuous phase (Type $C$ ). The occurrence of Type A or Type B amorphous phase separation in a glass causes the overall glass durability to be poor. The occurrence of Type $\mathrm{C}$ amorphous phase separation in a glass causes little or no impact on the overall glass durability. When the two separating phases are amorphous at the melt temperature but crystallize upon cooling the phenomena is called crystalline phase separation (CPS) and little is known about the impact of CPS on borosilicate waste glass durability. 
The type of phase separation found in borosilicate waste glasses in both the United States ${ }^{1,2,3}$ and in Europe ${ }^{4}$ has been shown to have an adverse and unpredictable effect on glass durability, the continuous phase is poorly durable and governs the durability response. For this reason, glass compositions that have a tendency to phase separate are excluded from consideration during waste processing in the SRS Defense Waste Processing Facility (DWPF). ${ }^{2,5}$ The phase separation exhibited by these low $(\leq 2.6 \mathrm{wt} \%)$ $\mathrm{P}_{2} \mathrm{O}_{5}$ containing borosilicate glasses is glass-in-glass or APS.

The type and scale of phase separation in phosphate containing borosilicate glasses, such as those proposed for the processing of high level waste at the INEEL, ${ }^{6}$ is different than the phase separation in the low $\mathrm{P}_{2} \mathrm{O}_{5}$ containing borosilicate waste glasses. The high concentrations of $\mathrm{P}_{2} \mathrm{O}_{5}$ in the INEEL wastes arise from one of the proposed processing options: dissolution of calcined waste followed by separation of the High Activity Waste (HAW) portion for vitrification. It is the separation process that concentrates the radionuclides in the HAW and adds large amounts of $\mathrm{P}_{2} \mathrm{O}_{5}$.

In phosphate-rich glasses, phosphate-rich regions form that are molten droplet like at the melt temperature: the glass cannot be cooled quickly enough to prevent the droplets from crystallizing into alkali phosphate phases. This type of phase separation is known as crystalline phase separation (CPS). The kinetics and the effects of the phase separation and composition on glass durability are different in CPS and APS. It is the purpose of this study to (1) investigate the effects of CPS in high $\mathrm{P}_{2} \mathrm{O}_{5}$ containing waste glasses on glass durability and to (2) recommend glass composition regions for future study if the high $\mathrm{P}_{2} \mathrm{O}_{5}$ separations process is chosen for INEEL.

\subsection{AMORPHOUS VS. CRYSTALLINE PHASE SEPARATION}

Glasses that contain significant amounts of two or more glass forming oxides are likely candidates for phase separation. Borosilicate glasses, although prone to APS, are widely used in the commercial glass industry. Compositions are selected to avoid the phase separated region or to take advantage of glass properties, such as thermal expansion, that are positively influenced by phase separation.

When alkali borosilicate glasses undergo APS, two liquid phases differing in composition usually develop that are not miscible in each other. Each compositional domain is usually enriched in one of the three major glass forming oxides, e.g. silica as $\left(\mathrm{SiO}_{4}\right)^{-4}$ tetrahedral units, boria as $\left(\mathrm{BO}_{4}\right)^{-5}$ tetrahedral units $\dagger$ or $\left(\mathrm{BO}_{3}\right)^{-3}$ trigonal units, $\ddagger$ or

\footnotetext{
$\dagger$ where $B$ is surrounded by four oxygen atoms or IV coordinated

$\ddagger$ where $B$ is surrounded by three oxygen atoms or III coordinated
} 
phosphorous oxide as $\left(\mathrm{PO}_{4}\right)^{-3}$ tetrahedral units. The competition for dominant structural role causes one or more of the three types of tetrahedral units to phase separate. In borosilicate glasses, $\left(\mathrm{PO}_{4}\right)^{-3}$ will separate first along with accompanying charge balancing cations. ${ }^{7}$ In general, the strong tendency toward phase separation can be anticipated from the competitive strong field strengths of the glass-formers $\mathrm{P}^{+5}=2.1, \mathrm{Si}^{4+}=1.57, \mathrm{~B}_{\mathrm{III}}^{3+}=1.63$ and $B_{I V}^{3+}=1.34$ (where III and IV refer to the coordination of the oxygen ions surrounding each $\mathrm{B}$ ). This causes phase separation in all the known binary systems, e.g., $\mathrm{SiO}_{2}-\mathrm{B}_{2} \mathrm{O}_{3}, \mathrm{SiO}_{2}-\mathrm{P}_{2} \mathrm{O}_{5}$, and $\mathrm{B}_{2} \mathrm{O}_{3}-\mathrm{P}_{2} \mathrm{O}_{5}{ }^{7}$

In ternary borosilicate glasses with little or no phosphate (glasses predominately in the $\mathrm{Na}_{2} \mathrm{O}-\mathrm{B}_{2} \mathrm{O}_{3}-\mathrm{SiO}_{2}$ system), a silica-rich phase often phase separates from an alkali-boron enriched domain. Tomozawa ${ }^{8}$ described three types of APS in alkali borosilicate glasses based on microstructure:

Type A: Both phases are continuous and interconnected (Figure 1)

- the durability of the glass is governed by the least durable phase

- usually formed as a result of spinodal decomposition

Type B: A silica-rich phase is dispersed as droplets in a non-durable (Figure 2)

- continuous matrix which is an alkali-borate phase

- usually formed by nucleation and growth

Type C: An alkali-borate phase is dispersed as droplets in a continuous matrix of silica-rich phase (Figure 3)

- the durability of the glass is governed by the continuous phase that is highly durable

- usually formed by nucleation and growth.

Complex combinations of these types of APS can occur in the same glass, e.g., glasses are known that undergo "primary" APS of Type B while the borate-rich continuous matrix undergoes a "secondary" Type B APS. Such complex phase separation can result in glasses with 3 or more different amorphous phases ${ }^{7}$ each with a different durability response. Indeed, Figure 2 shows "primary" phase separation of Type B (large $\mathrm{SiO}_{2}$-rich droplets in a sodium borate rich continuous matrix). However, the borate-rich continuous matrix has undergone a "secondary" phase separation of Type $\mathrm{B}$ and smaller $\mathrm{SiO}_{2}$-rich droplets can be seen within the borate-rich matrix. Likewise, in Figure 3 the "primary" phase separation is Type $\mathrm{C}$ and large sodium-borate rich droplets have formed in a durable $\mathrm{SiO}_{2}$-rich continuous matrix phase. There are also smaller Type $\mathrm{C}$ sodium-borate 


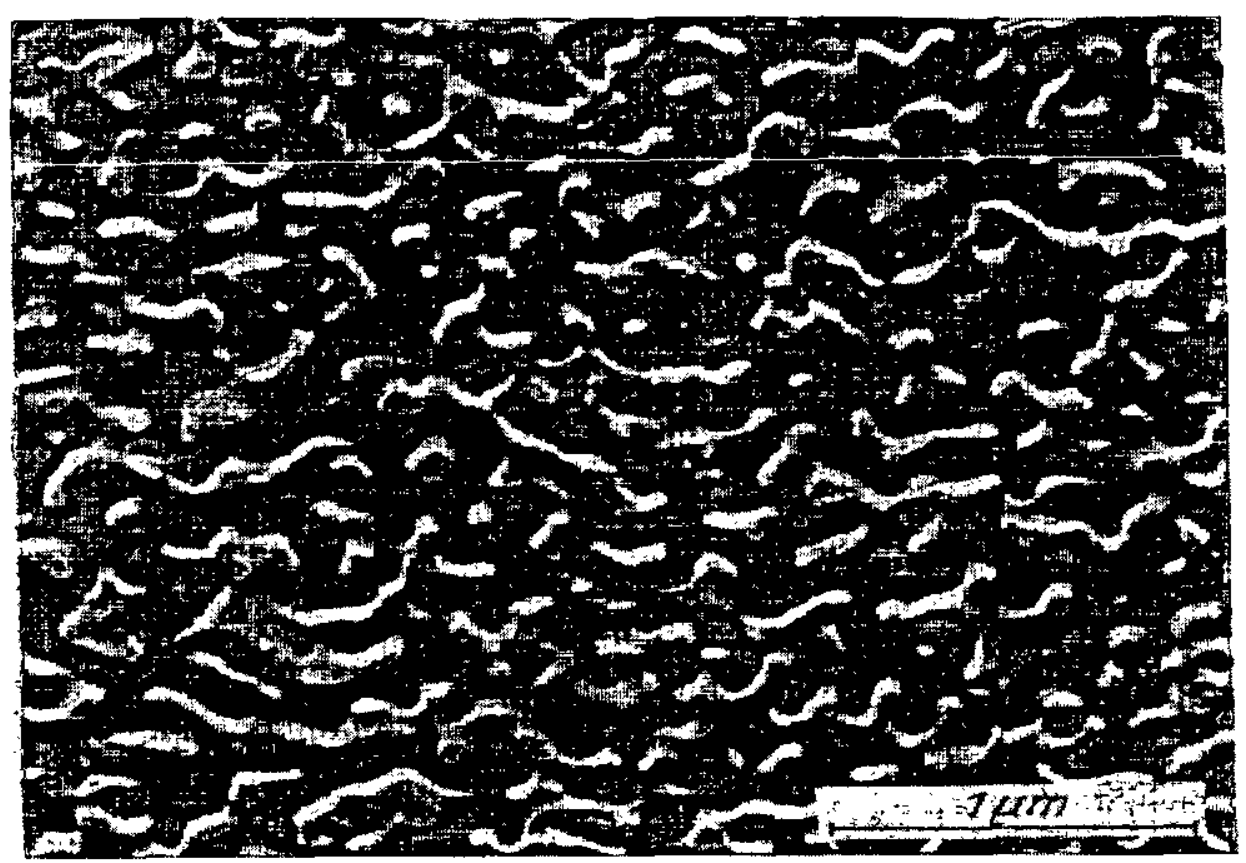

Figure 1. Example of Type A phase separation (described above) in sodium borosilicate glass.

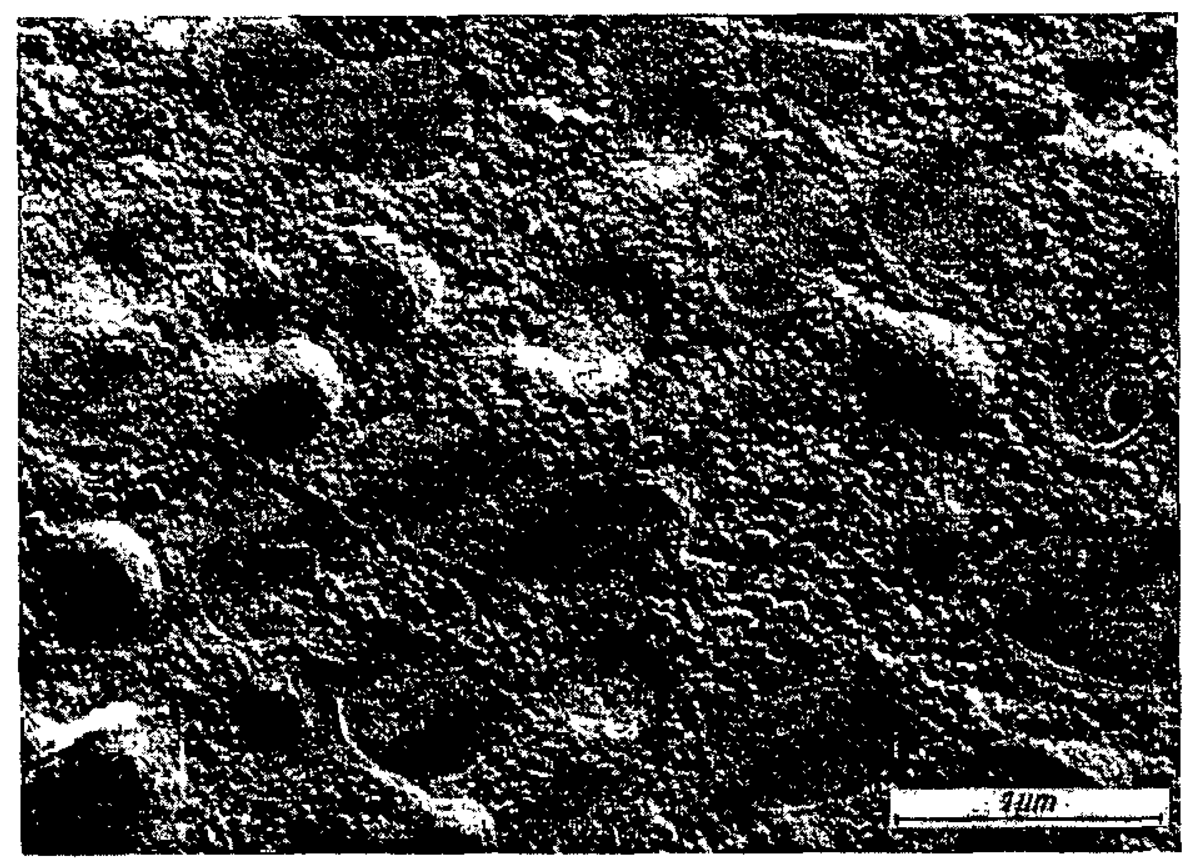

Figure 2. Example of Type B phase separation (described above) in sodium borosilicate glass. 


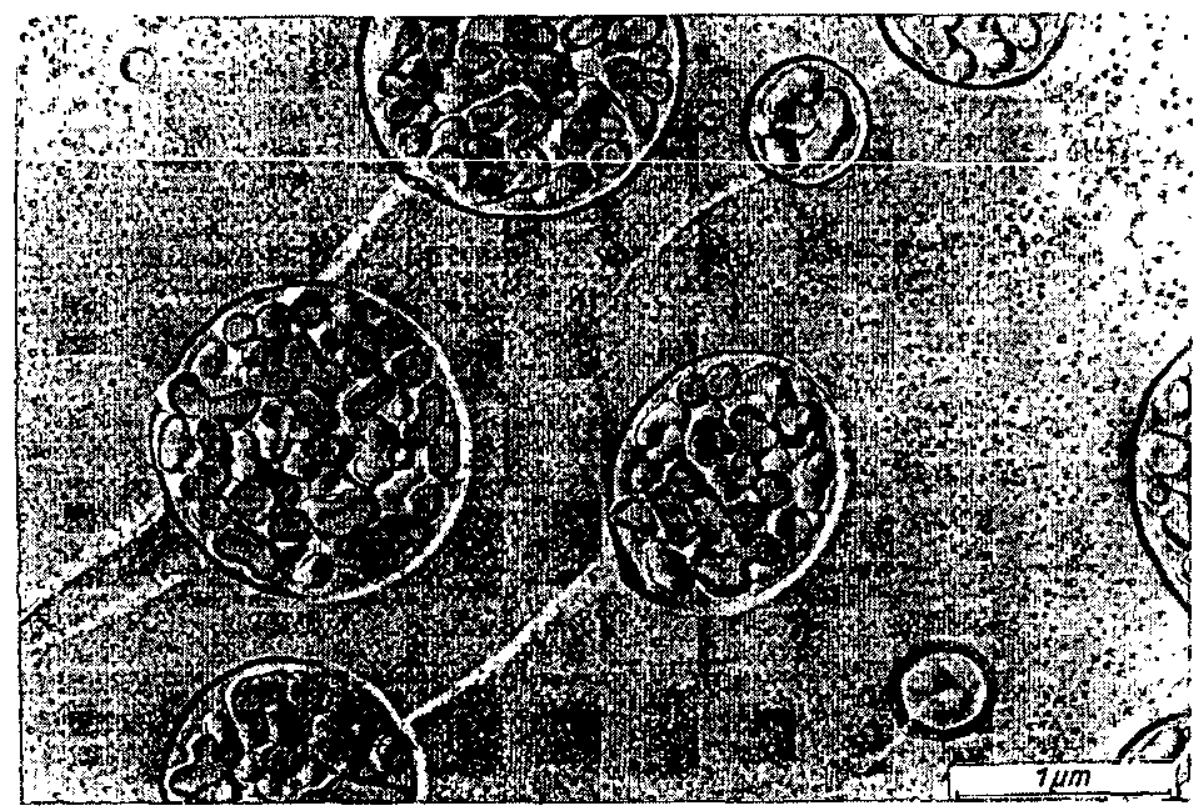

Figure 3. Example of Type $\mathrm{C}$ phase separation (described above) in sodium borosilicate glass. ${ }^{4}$

rich precipitates in the continuous $\mathrm{SiO}_{2}$-rich matrix formed by "secondary" phase separation. In addition, the large sodium-rich droplets have undergone a Type B "secondary" phase separation. The resulting glass has 4 different phases - three Type C separated phases and one Type B separated phase.

In phosphate containing borosilicate glasses all three major glass formers are present in structural tetrahedral or trigonal groups, e.g. $\left(\mathrm{SiO}_{4}\right)^{-4},\left(\mathrm{BO}_{4}\right)^{-5},\left(\mathrm{BO}_{3}\right)^{-3}$, and $\left(\mathrm{PO}_{4}\right)^{-3}$. The competition for dominant structural role causes the cation with the highest field strength, $\mathrm{P}^{+5}$, to dominate the de-mixing process. If the difference in the field strengths $(\Delta \mathrm{F})$ between the major glass forming (structural) elements exceeds 0.3 , then the phase separation will be of a crystalline and not amorphous nature according to Dietzel, ${ }^{7}$ e.g. the differences between $\mathrm{B}$ and $\mathrm{P}$ and $\mathrm{Si}$ and $\mathrm{P}$ exceed the limit of 0.3 while the $\Delta \mathrm{F}$ difference between $\mathrm{B}$ and $\mathrm{Si}$ is only 0.06 . Therefore, the phase separation in phosphate systems is of a crystalline nature, CPS. Glasses in these phosphate systems phase separate to crystalline or partially crystalline solids, depending on quench rate (Figures 4 and 5). 


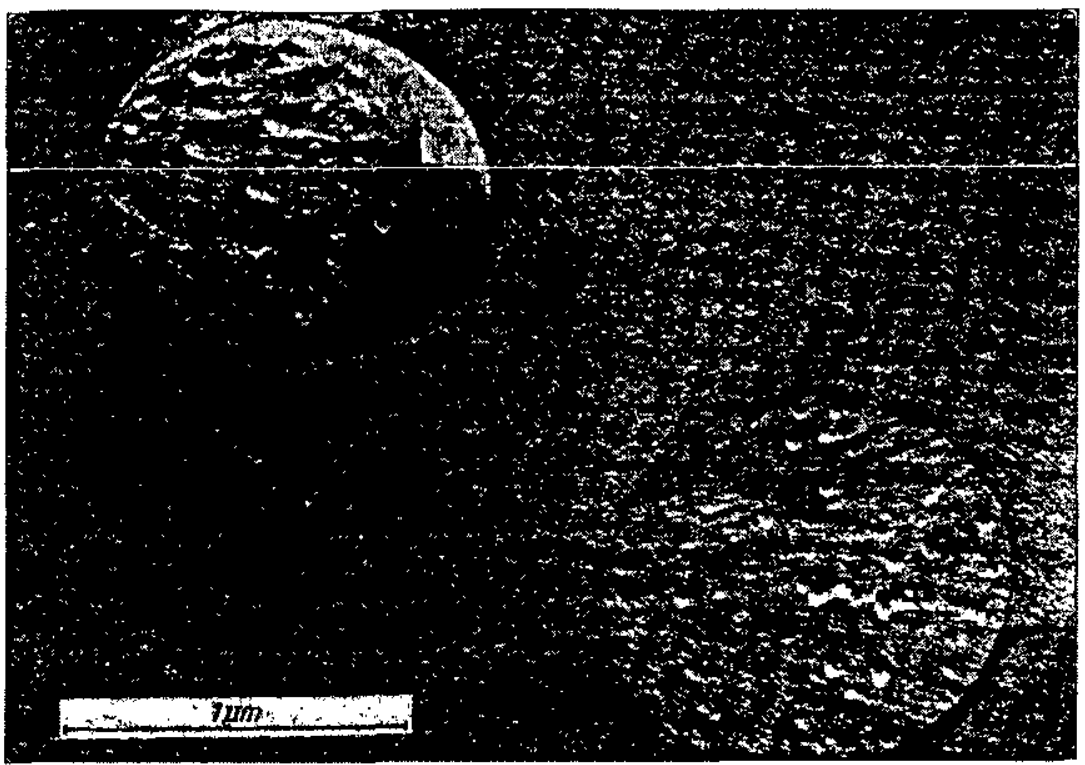

Figure 4. CaO- $\mathrm{P}_{2} \mathrm{O}_{5}$ rich droplets which have crystallized in a phosphate rich borosilicate glass (from Vogel, ref.').

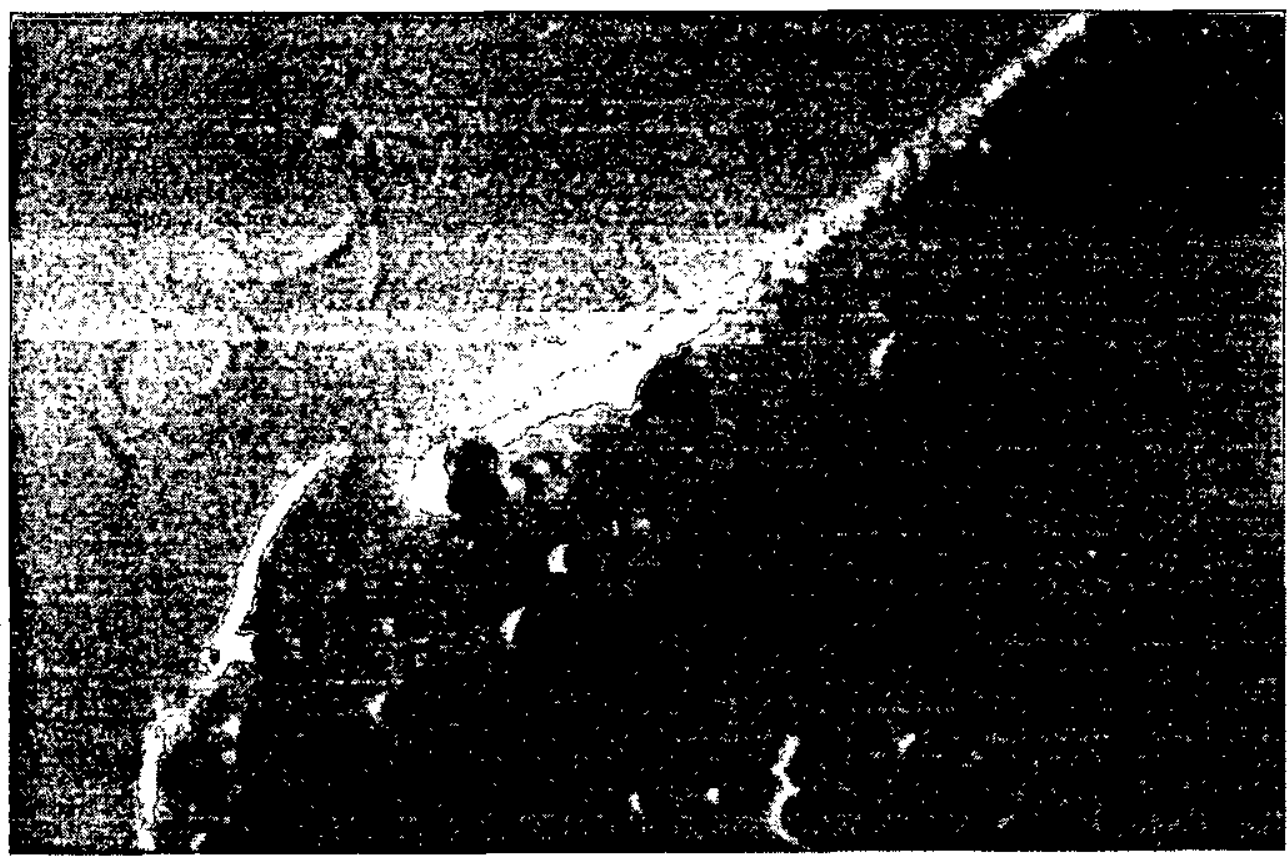

Figure 5. $\mathrm{Li}_{3} \mathrm{PO}_{4}$ crystalline droplets in an SRS phosphate rich borosilicate glass (from Jantzen ${ }^{9}$ ). 


\subsection{HISTORICAL BACKGROUND}

The earliest work reported on CPS in nuclear waste glasses was that of Jantzen ${ }^{9}$ in 1986 (Table I; Figure 5): crystalline $\mathrm{Li}_{3} \mathrm{PO}_{4}$ was found to preferentially form as droplets in $\mathrm{Li}_{2} \mathrm{O}$ containing borosilicate waste glasses when the solubility limit of $\mathrm{P}_{2} \mathrm{O}_{5}$ in the glass was exceeded $\left(\geq 2.6 \mathrm{wt} \% \mathrm{P}_{2} \mathrm{O}_{5}\right)$. Other studies (Table I) showed that $\mathrm{Na}_{3}\left(\mathrm{PO}_{4}\right)_{2}$ crystallized when $\mathrm{Na}_{2} \mathrm{O}$ was the only alkali oxide present while $\mathrm{Ca}_{3}\left(\mathrm{PO}_{4}\right)_{2}$ or the mixed phosphate phase, $\mathrm{Na}_{2} \mathrm{Ca}_{4}\left(\mathrm{PO}_{3}\right)_{2} \mathrm{SiO}_{4}$ crystallized when both $\mathrm{Na}_{2} \mathrm{O}$ and $\mathrm{CaO}$ oxides were present. The $\mathrm{P}_{2} \mathrm{O}_{5}$ solubility limits in each study were in the 2.5-3.0 wt\% range depending on the particular alkali and alkaline earth species present.

Table I. Phase Separation in Borosilicate Glasses Containing $\mathrm{P}_{2} \mathrm{O}_{5}$

\begin{tabular}{|c|c|c|c|c|c|c|c|}
\hline $\begin{array}{l}\mathrm{P}_{2} \mathrm{O}_{5} \\
(\mathrm{w}+\%)\end{array}$ & $\begin{array}{l}\mathrm{B}_{2} \mathrm{O}_{3} \\
(\mathrm{ivo} \%) \\
\end{array}$ & $\begin{array}{l}\Sigma \mathrm{M}_{2} \mathrm{O}^{2} \\
(\mathbf{w i t} \%) \\
\end{array}$ & $\begin{array}{l}\mathrm{SiO}_{2} / \mathrm{h} \\
\mathrm{B}_{2} \mathrm{O}_{3}\end{array}$ & $\begin{array}{l}\text { Major } \\
\text { Alkali }\end{array}$ & CPSPhase & $\begin{array}{l}\text { GlassiMelt } \\
\text { Temp. (CO) }\end{array}$ & Rë́f \\
\hline Boros & cate 6 & $\operatorname{asses} \mathrm{C}$ & itaining & $\mathrm{K}_{2} \mathrm{O} \mathrm{O}$ & 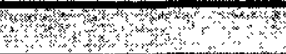 & & 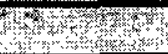 \\
\hline$\geq 2.6$ & 7.2 & $\sim 16$ & 7.64 & $\begin{array}{c}\mathrm{Na}_{2} \mathrm{O} \\
+ \\
\mathrm{Li}_{2} \mathrm{O}\end{array}$ & $\mathrm{Li}_{3} \mathrm{PO}_{4}$ & $\begin{array}{l}\text { DWPF } \\
165 @ \\
1150^{\circ} \mathrm{C}\end{array}$ & $\begin{array}{l}\text { Jantzen } \\
(1986)^{9}\end{array}$ \\
\hline $\begin{array}{l}3.33- \\
7.33\end{array}$ & $\begin{array}{c}13.06 \\
- \\
13.63\end{array}$ & $\begin{array}{l}10.92 \\
- \\
11.15\end{array}$ & 3.83 & $\begin{array}{l}\mathrm{Li}_{2} \mathrm{O} \\
+ \\
\mathrm{Na}_{2} \mathrm{O}\end{array}$ & $\begin{array}{r}\mathrm{Li}_{3} \mathrm{PO}_{4} \\
+\mathrm{Cr}_{2} \mathrm{O}_{3} \\
+\mathrm{AlPO}_{4}\end{array}$ & $\begin{array}{c}\mathrm{PFP}^{+t} \\
\text { Waste @ } \\
1150^{\circ} \mathrm{C}\end{array}$ & $\begin{array}{c}\text { Langow- } \\
\text { ski } \\
(1996)^{10}\end{array}$ \\
\hline Borosi & cate G & asses W & thout L & & & & \\
\hline $\begin{array}{c}2.68- \\
5.05\end{array}$ & $\begin{array}{l}17.6- \\
19.5\end{array}$ & $\begin{array}{c}16- \\
17.66\end{array}$ & $\begin{array}{l}3.06- \\
3.47\end{array}$ & $\mathrm{Na}_{2} \mathrm{O}$ & $\begin{array}{c}\mathrm{SiO}_{2} \\
\text { (Cristobalite }+ \\
\text { Tridymite) }\end{array}$ & $\begin{array}{c}\mathrm{Na}_{2} \mathrm{O}- \\
\mathrm{B}_{2} \mathrm{O}_{3}- \\
\mathrm{SiO}_{2} \\
\text { (a) } 1150^{\circ} \mathrm{C}\end{array}$ & $\begin{array}{l}\text { Cozzi, } \\
(1998)^{11}\end{array}$ \\
\hline$\sim 3$ & 5.00 & 20.33 & 11.36 & $\begin{array}{l}\mathrm{Na}_{2} \mathrm{O} \\
+\mathrm{CaO} \\
+\mathrm{K}_{2} \mathrm{O}\end{array}$ & $\begin{array}{c}\mathrm{Na}_{3} \mathrm{PO}_{4} \\
\text { and } \\
\mathrm{Na}_{2} \mathrm{Ca}_{4}\left(\mathrm{PO}_{3}\right)_{2} \mathrm{~S} \\
\mathrm{iO}_{4}\end{array}$ & $\begin{array}{l}\text { LLW @ } \\
1300^{\circ} \mathrm{C}\end{array}$ & $\begin{array}{l}\text { Crichton } \\
\text { et. al. } \\
(1995)^{12}\end{array}$ \\
\hline$\sim 2.5$ & 17 & 6.5 & 4.16 & $\begin{array}{l}\mathrm{Na}_{2} \mathrm{O} \\
+\mathrm{CaO}\end{array}$ & $\mathrm{Ca}_{3}\left(\mathrm{PO}_{4}\right)_{2}$ & $\begin{array}{l}\text { Comm. } \\
\text { Pyrex }\end{array}$ & $\begin{array}{l}\text { Vogel, } \\
(1985)^{7}\end{array}$ \\
\hline
\end{tabular}

$\ddagger$ where $\mathrm{M}_{2} \mathrm{O}=\Sigma \mathrm{Na}_{2} \mathrm{O}+\mathrm{Li}_{2} \mathrm{O}+\mathrm{K}_{2} \mathrm{O}+\mathrm{Cs}_{2} \mathrm{O} \quad$ \$\$Pu Finishing Plant 


\subsection{EXPERIMENTAL}

Glasses from the INEEL waste glass composition variability study ${ }^{\text {Error! Bookmark not defined. }}$ were examined by $x$-ray diffraction (XRD) analyses in order to identify the CPS phases present. Analyses performed in this study are shaded in Table II. The INEEL database containing 44 "as batched" glass compositions was combined with a mixed waste treatability study database containing 51 analyzed actual mixed waste glasses from vitrification of SRS M-Area wastes (8 from pilot scale runs and 43 crucible studies). The M-Area data has here-to-fore not been previously published. All the previously unpublished data is shaded in Tables II, III, and IV. The INEEL glasses had all been melted in platinum crucibles at $1150^{\circ} \mathrm{C}$ except glass IG1-33 which was melted at $1450^{\circ} \mathrm{C}$. The INEEL glasses had been rapidly quenched by pouring onto a steel block. The M-Area waste glasses had been melted in $99.9 \% \mathrm{Al}_{2} \mathrm{O}_{3}$ crucibles since this was high $\mathrm{Al}_{2} \mathrm{O}_{3}$ containing waste there was no interaction with the ceramic crucibles. The glasses had been cooled in the crucibles undergoing a somewhat slower quench than the INEEL glasses. The remainder of the M-Area glasses were poured from a pilot scale melter at $1150^{\circ} \mathrm{C}$. The glass was poured into stainless steel cans about 8 " in diameter and 12" deep. This glass underwent the slowest quench rate of all the glasses studied. Details of the fabrication of the INEEL, M-Area, and other glasses is given in Table II. The CPS phases in the M-Area waste glasses had been analyzed by XRD.

The INEEL waste glasses contained up to $13.5 \mathrm{wt} \% \mathrm{P}_{2} \mathrm{O}_{5}$ while the M-Area glasses contained a maximum of $\sim 4 \mathrm{wt} \% \mathrm{P}_{2} \mathrm{O}_{5}$. The INEEL glasses contained no $\mathrm{U}_{3} \mathrm{O}_{8}$ while the M-Area glasses contained up to $5.66 \mathrm{wt} \% \mathrm{U}_{3} \mathrm{O}_{8}$. The INEEL waste glasses contained up to $14 \mathrm{wt} \% \mathrm{ZrO}_{2}$ because some of the INEEL waste calcines are high in $\mathrm{ZrO}_{2}$. The M-Area wastes contained no $\mathrm{ZrO}_{2}$ but this component was added to enhance glass durability (maximum level of $2 \mathrm{wt} \%$ ). The $\mathrm{Al}_{2} \mathrm{O}_{3}$ ranged from 4 to $15 \mathrm{wt} \%$ in the INEEL study and from 12.75 to $29 \mathrm{wt} \%$ in the M-Area glasses. Concentrations of $\mathrm{Na}_{2} \mathrm{O}$ in the INEEL waste glasses spanned between 5 and $21.3 \mathrm{wt} \%$ while the M-Area glasses spanned between 6.8 and $27.09 \mathrm{wt} \%$. Lastly, 4.37 to $15 \mathrm{wt} \% \mathrm{~B}_{2} \mathrm{O}_{3}$ was used as a glass forming flux in the high $\mathrm{ZrO}_{2}$ containing INEEL waste glasses while 5.19 to $32.62 \mathrm{wt} \% \mathrm{~B}_{2} \mathrm{O}_{3}$ was used as a flux in the high $\mathrm{Al}_{2} \mathrm{O}_{3}$ containing M-Area waste. A comparison of the compositional ranges spanned by the borosilicate M-Area and INEEL studies is given in Table V. Ten of the M-Area glasses are soda-lime-silicate glasses. Individual measured and "as batched" glass compositions are given in Table III.

The durability of the M-Area and INEEL waste glasses were tested using ASTM C1285-97 (Method A for the INEEL glasses and Method B for the M-Area glasses at the reference 7 day, $90^{\circ} \mathrm{C}$, and 1:10 ratio of mass solids: mass solution). The crushed glass tested was sieved to $-100+200$ mesh and the fines washed first in water and then 
in ethanol. While it is recognized in the ASTM C1285-97 procedure that removal of the fines adhering to the $-100+200$ mesh fraction in water can preferentially remove soluble components from the glass, comparison testing by washing in ethanol only is in progress. A mathematical approach is used in Section 6.0 to determine if any constituents of interest were preferentially removed due to the water wash. Measured normalized release rates are given in Table IV.

The 85 borosilicate glasses in the INEEL and M-Area studies spanned a wide composition range in terms of the alkalis, $\mathrm{Al}_{2} \mathrm{O}_{3}, \mathrm{ZrO}_{2}, \mathrm{P}_{2} \mathrm{O}_{5}$ and $\mathrm{U}_{3} \mathrm{O}_{8}$. Another 8 data points from $\mathrm{Cozzi}^{1 \mathrm{l}}$ on simple three and four component sodium borosilicate glasses with and without $\mathrm{P}_{2} \mathrm{O}_{5}$ were added to the database (see Tables II, III, and IV). Only 6 of these 8 glasses could be used to assess glass durability since PCT measurements were missing for 2 of the glasses. The 4 waste glasses from the work of Langowski, $\mathrm{Li}$, et. al ${ }^{10}$ could not be used because durability data was not available. Only "as-batched" glass compositions were available from the Cozzi and Langowski studies. A total of 91 borosilicate glasses (41 from M-Area, 44 from INEEL and 6 from the Cozzi study) of the 107 glasses given in Tables II, III, and IV compose the database used in this study. The 10 soda-lime silica glasses, 2 Cozzi glasses, and 4 Langowski glasses are omitted from further consideration.

\subsection{TYPES OF CRYSTALLINE PHASE SEPARATION (CPS) OBSERVED}

The CPS phases identified in the glasses are summarized in Table V. Eight of $41 \mathrm{M}-$ Area glasses crystallized $\mathrm{Li}_{3} \mathrm{PO}_{4}$ as the primary crystalline phase and 3 crystallized $\mathrm{Li}_{3} \mathrm{PO}_{4}$ along with an additional silicate or $\mathrm{SiO}_{2}$. Only one glass, $\mathrm{MN}-5$ exceeded its solubility limit and crystallized $\alpha-\mathrm{Al}_{2} \mathrm{O}_{3}$. Glass MN-26 appeared to have incompletely reacted and sodium carbonate (a glass forming additive) was identified. Twenty four of the 44 INEEL glasses crystallized $\mathrm{Li}_{3} \mathrm{PO}_{4}$ and/or $\mathrm{Li}_{2} \mathrm{NaPO}_{4}$. One glass crystallized $\mathrm{Li}_{2} \mathrm{MgP}_{2} \mathrm{O}_{7}$ and another crystallized $\mathrm{Na}_{2} \mathrm{ZrSiO}_{5}$. Only one glass, IG1-30 appeared to have exceeded its solubility limit and crystallized $\mathrm{ZrO}_{2}$.

Many of the glasses appeared visually to be amorphous and were found to be amorphous during XRD analyses (Table II). However, it was suspected that several of the glasses had undergone amorphous phase separation (APS) that would not have been observed by XRD. Of the glasses examined by TEM, those classified as inhomogeneous were found to have crystalline phases. 
Table $\mathbb{I I}$. Database of $\mathbb{B}$ orosilicate $\mathbb{G}$ lasses $\mathbb{C o n t a i n i n g} \mathbb{P}_{2} \mathbb{O}_{5}:$ Fabrication Methods and Homogeneity

\begin{tabular}{|c|c|c|c|c|c|}
\hline Sampie & $\begin{array}{l}\text { ivelt } \\
\text { Temperature } \\
\left({ }^{\circ} \mathrm{C}\right)\end{array}$ & $\begin{array}{l}\text { Weit /Quencin } \\
\text { Nechnique }\end{array}$ & $\begin{array}{l}\text { Type of } \\
\text { Glass }\end{array}$ & $\begin{array}{l}\text { Auralyicic } \\
\text { Booi Úsed }\end{array}$ & $\begin{array}{l}\text { Dystaline } \\
\text { Phase } \\
\text { Separated }\end{array}$ \\
\hline IG1-01 & 1150 & Crucible/Patty & Borosilicate & TEM/XRD & $\mathrm{Li}_{3} \mathrm{PO}_{4}$ \\
\hline IG1-02 & 1150 & Crucible/Patty & Borosilicate & XRD & $\mathrm{Li}_{3} \mathrm{PO}_{4}$ \\
\hline IG1-03 & 1150 & Crucible/Patty & Borosilicate & XRD & $\mathrm{Na}_{3} \mathrm{PO}_{4}$ \\
\hline IG1-04 & 1150 & Crucible/Patty & Borosilicate & TEM/XRD & $\mathrm{Li}_{3} \mathrm{PO}_{4}$ \\
\hline IG1-05 & 1150 & Crucible/Patty & Borosilicate & TEM/XRD & $\mathrm{Li}_{2} \mathrm{MgP} \mathrm{P}_{2} \mathrm{O}_{7}$ \\
\hline IG1-06 & 1150 & Crucible/Patty & Borosilicate & $\mathrm{XRD}$ & Amorphous \\
\hline IG1-07 & 1150 & Crucible/Patty & Borosilicate & XRD & Amorphous \\
\hline IGI-08 & 1150 & Crucible/Patty & Borosilicate & TEM & $\mathrm{Li}_{3} \mathrm{PO}_{4}$ \\
\hline IGI-09 & 1150 & Crucible/Patty & Borosilicate & TEM/XRD & $\mathrm{Li}_{2} \mathrm{NaPO}_{4}$ \\
\hline IG1-10 & 1150 & Crucible/Patty & Borosilicate & XRD & Amorphous \\
\hline IGI-11 & 1150 & Crucible/Patty & Borosilicate & XRD & Amorphous \\
\hline IG1-12 & 1150 & Crucible/Patty & Borosilicate & TEM & Amorphous \\
\hline IG1-13 & 1150 & Crucible/Patty & Borosilicate & TEM & Amorphous \\
\hline IG1-14 & 1150 & Crucible/Patty & Borosilicate & TEM/XRD & Amorphous \\
\hline IG1-15 & 1150 & Crucible/Patty & Borosilicate & $\mathrm{XRD}$ & $\mathrm{Li}_{3} \mathrm{PO}_{4}$ \\
\hline IG1-16 & 1150 & Crucible/Patty & Borosilicate & TEM & Amorphous \\
\hline IG1-17 & 1150 & Crucible/Patty & Borosilicate & TEM/XRD & $\mathrm{Li}_{2} \mathrm{NaPO}_{4}$ \\
\hline IG1-18 & 1150 & Crucible/Patty & Borosilicate & $\mathrm{XRD}$ & $\mathrm{Li}_{3} \mathrm{PO}_{4}$ \\
\hline IG1-19 & 1150 & Crucible/Patty & Borosilicate & XRD & $\operatorname{Lij}_{13}$ \\
\hline IG1-20 & 1150 & Crucible/Patty & Borosilicate & TEM & $\mathrm{L}_{3} \mathrm{PO}_{4}$ \\
\hline IG1-21 & 1150 & Crucible/Patty & Borosilicate & TEM/XRD & $\mathrm{Li}_{3} \mathrm{PO}_{4}$ \\
\hline IG1-22 & 1150 & Crucible/Patty & Borosilicate & XRD & $\mathrm{Li}_{3} \mathrm{PO}_{4}$ \\
\hline IG1-23 & 1150 & Crucible/Patty & Borosilicate & XRD & $\mathrm{Li}_{3} \mathrm{PO}_{4}$ \\
\hline IG1-24 & 1150 & Crucible/Patty & Borosilicate & TEM/XRD & $\begin{array}{c}\mathrm{Li}_{3} \mathrm{PO}_{4} \\
\mathrm{Na}_{2} \mathrm{ZrSiO}_{5}\end{array}$ \\
\hline IG1-25 & 1150 & Crucible/Patty & Borosilicate & $\mathrm{XRD}$ & $\mathrm{Li}_{3} \mathrm{PO}_{4}$ \\
\hline IG1-26 & 1150 & Crucible/Patty & Borosilicate & TEM/XRD & $\mathrm{Li}_{3} \mathrm{PO}_{4}$ \\
\hline IG1-27 & 1150 & Crucible/Patty & Borosilicate & $\mathrm{XRD}$ & $\mathrm{Li}_{3} \mathrm{PO}_{4}$ \\
\hline IG1-28 & 1150 & Crucible/Patty & Borosilicate & XRD & $\mathrm{Li}_{3} \mathrm{PO}_{4}$ \\
\hline IG1-29 & 1150 & Crucible/Patty & Borosilicate & $\mathrm{XRD}$ & Ámorphous \\
\hline IG1-30 & 1150 & Crucible/Patty & Borosilicate & $\mathrm{XRD}$ & $\mathrm{ZrO}_{2}(\mathrm{tr})$ \\
\hline IG1-31 & 1150 & Crucible/Patty & Borosilicate & XRD & Amorphous \\
\hline$\overline{\text { IG1-32 }}$ & 1150 & Crucible/Patty & Borosilicate & XRD & $\mathrm{Li}_{3} \mathrm{PO}_{4}$ \\
\hline IG1-33 & 1450 & Crucible/Patty & Borosilicate & XRD & Amorphous \\
\hline IGI-34 & 1150 & Crucible/Patty & Borosilicate & TEM/XRD & $\mathrm{Li}_{3} \mathrm{PO}_{4}$ \\
\hline IG1-35 & 1150 & Crucible/Patty & Borosilicate & TËM/XRD & Amorphous \\
\hline IG1-36 & 1150 & Crucible/Patty & Borosilicate & XRD & Amorphous \\
\hline IG1-37 & 1150 & Crucible/Patty & Borosilicate & TEM/XRD & Amorphous \\
\hline IGI-38 & 1150 & Crucible/Patty & Borosilicate & TEM/XRD & Amorphous \\
\hline IG1-39 & 1150 & Crucible/Patty & Borosilicate & TEM/XRD & Amorphous \\
\hline IG1-40 & 1150 & Crucible/Patty & Borosilicate & XRD & $\mathrm{Li}_{3} \mathrm{PO}_{4}$ \\
\hline
\end{tabular}




\begin{tabular}{|c|c|c|c|c|c|}
\hline Sampie & $\begin{array}{l}\text { iVielt } \\
\text { Temperature } \\
\left({ }^{\circ} \mathrm{C}\right)\end{array}$ & $\begin{array}{l}\text { Welt /Quench } \\
\text { Pechnique }\end{array}$ & $\begin{array}{l}\text { Gype o? } \\
\text { Giass }\end{array}$ & $\begin{array}{l}\text { Analyljc } \\
\text { Cooi Used }\end{array}$ & $\begin{array}{l}\text { C-ystalline } \\
\text { Phase } \\
\text { Separated }\end{array}$ \\
\hline IGI-41 & 1150 & Crucible/Patty & Borosilicate & XRD & $\mathrm{Li}_{3} \mathrm{PO}_{4}$ \\
\hline IGI-42 & 1150 & Crucible/Patty & Borosilicate & TEM/XRD & $\mathrm{Li}_{3} \mathrm{PO}_{4}$ \\
\hline IG1-43 & 1150 & Crucible/Patty & Borosilicate & $\overline{\mathrm{TEM} / \mathrm{XRD}}$ & Amorphous \\
\hline IGI-44 & 1150 & Crucible/Patty & Borosilicate & TEM/XRD & $\mathrm{Li}_{3} \mathrm{PO}_{4}$ \\
\hline $\mathrm{MN}-1$ & 1400 & Crucible/In Crucible & Borosilicate & $\overline{X R D}$ & Amorphous \\
\hline MN-2 & 1260 & Crucibie/̆n Crucible & Borosilicate & XRD & Amorphous \\
\hline $\mathrm{MN}-3$ & 1150 & Crucible/In Crucible & Borosilicate & $\overline{\mathrm{XRD}}$ & Ámorphous \\
\hline $\mathrm{MN}-4$ & 1400 & Crucible/In Crucible & Borosilicate & $\mathrm{XRD}$ & Ámorphous \\
\hline $\mathrm{MN}-5$ & 1300 & Crucible//n Crucible & Borosílicate & $\overline{X R D}$ & $\alpha-\mathrm{A}_{2} \mathrm{O}_{3}$ \\
\hline MN-6 & 1150 & Crucible/In Crucible & $\overline{\text { Borosilicate }}$ & $\overline{\mathrm{XRD}}$ & Ämorphous \\
\hline $\mathrm{MN}-7$ & 1400 & Crucible/In Crucible & Borosilicate & $\mathrm{XRD}$ & Amorphous \\
\hline MN-8 & 1150 & Crucible/In Crucible & Borosilicate & $\overline{X R D}$ & Ámorphous \\
\hline$\overline{M N}-9 \bar{A}$ & 1200 & Crucible/In Crucible & Borosilicate & $\overline{\mathrm{XRD}}$ & Amorphous \\
\hline MN-9B & 1200 & Crucible/In Crucible & Borosilicate & $\overline{X R \bar{D}}$ & Amorphous \\
\hline$\overline{M N}-10$ & 1200 & Crucible/ln Crucible & Borosilicate & $X \overline{R D}$ & $\mathrm{Li}_{3} \mathrm{PO}_{4}$ \\
\hline $\mathrm{MN}-11$ & $\pi 150$ & Crucible/tn Crucible & Borosilicate & $\overline{X R D}$ & $\mathrm{Li}_{3} \mathrm{PO}_{4}$ \\
\hline$\overline{M N}-12$ & 1150 & Crucible/In Crucible & Borosilicate & $\overline{X R D}$ & Arnorphous \\
\hline $\mathrm{MN}-13$ & 1150 & Crucible/ln Crucible & Borosilicate & $\overline{X R D}$ & Amorphous \\
\hline $\mathrm{MN}-14$ & 1150 & Crucible/In Crucible & Borosilicate & XRD & $\mathrm{Li}_{3} \mathrm{Mg}_{0.1} \mathrm{SiO}_{1}$ \\
\hline $\mathrm{MN}-15$ & 1200 & Crucible/In Crucible & Borosilicate & $\overline{X R D}$ & Amorphous \\
\hline $\mathrm{MN}-16$ & 1150 & Crucible/In Crucible & Borosilicate & $\overline{X R D}$ & Amorphous \\
\hline $\mathrm{MN}-17$ & $\longdiv { 1 3 5 0 }$ & Crucible/ln Crucible & Soda-Lime & $\overline{\mathrm{XRD}}$ & $\begin{array}{c}\mathrm{Li}_{3} \mathrm{PO}_{4} \\
\mathrm{Ca}_{3} \mathrm{Mg}\left(\mathrm{SiO}_{4}\right)_{2}\end{array}$ \\
\hline MN-18 & 1150 & Crucible/In Crucible & Soda-Lime & $\mathrm{XRD}$ & Amorphous \\
\hline MIN-19 & 1150 & Crucible/ln Crucible & Soda-Lime & $\overline{X R D}$ & Amorphous \\
\hline MN-20 & 1200 & Crucible/In Crucible & Soda-Lime & XRD & $\mathrm{Li}_{3} \mathrm{PO}_{4}$ \\
\hline MN-21 & 1150 & Crucible/In Crucible & Soda-Lime & XRD & Amorphous \\
\hline $\mathrm{MN}-22$ & 1150 & Crucible/In Crucible & Soda-Lime & XRD & $\mathrm{Li}_{2.8} \mathrm{Mg}_{0.6} \mathrm{SiO}_{4}$ \\
\hline $\mathrm{MN}-23$ & 1400 & Crucible/In Crucible & Soda-Lime & $\overline{\mathrm{XRD}}$ & $\begin{array}{r}\mathrm{Na}_{2} \mathrm{Ca}_{3} \mathrm{Al}_{2}(\mathrm{P} \\
\left.\mathrm{O}_{4}\right)_{2}\left(\mathrm{SiO}_{4}\right)_{2}\end{array}$ \\
\hline $\mathrm{MN}-24$ & 1400 & Crucible/In Crucible & Soda-Lime & $\mathrm{XRD}$ & $\mathrm{Li}_{3} \mathrm{PO}_{4}$ \\
\hline $\mathrm{MN}-25$ & 1150 & Crucible/In Crucible & Soda-Lime & XRD & Amorphous \\
\hline${ }^{8 N}-26$ & 1150 & Crucible/In Crucible & Soda-Lime & $\overline{\mathrm{XRD}}$ & $\mathrm{Na}\left(\mathrm{HCO}_{3}\right)_{2}$ \\
\hline MHSi-1 & 1400 & Crucible/In Crucible & Borosilicate & XRD & Amorphous \\
\hline MHSi-2 & 1300 & Crucible/In Crucible & Borosilicate & XRD & Amorphous \\
\hline MHSi-3 & 1150 & Crucible/In Crucible & Borosilicate & $\overline{\mathrm{XRD}}$ & Amorphous \\
\hline MHSi-4 & 1400 & Crucible/In Crucible & Borosilicate & XRD & Amorphous \\
\hline MHSi-5 & 1150 & Crucible/In Crucible & Borosilicate & XRD & Amorphous \\
\hline MHSi-6 & 1200 & Crucible/In Crucible & Borosilicate & XRD & Amorphous \\
\hline MHSi-11 & 1350 & Crucible/In Crucible & & XRD & $\mathrm{LiAlO}_{2}$ \\
\hline MLSi-1 & 1300 & Crucible/In Crucible & & XRD & Amorphous \\
\hline MLSi-2 & 1150 & Crucible/In Crucible & Borosilicate & $\mathrm{XRD}$ & Amorphous \\
\hline MLSi-3 & 1400 & Crucible/In Crucible & Borosilicate & XRD & Amorphous \\
\hline MLSi-4 & 1150 & Crucible/In Crucible & Borosilicate & XRD & Amorphous \\
\hline
\end{tabular}




\begin{tabular}{|c|c|c|c|c|c|}
\hline Sample & $\begin{array}{l}\text { Meit } \\
\text { Oemperature } \\
\left({ }^{\circ} \mathrm{C}\right)\end{array}$ & $\begin{array}{l}\text { iVelê /Quencin } \\
\text { Technique }\end{array}$ & $\begin{array}{l}\text { Type ox } \\
\text { Glass }\end{array}$ & $\begin{array}{l}\text { Anaiyite } \\
\text {. ool Used }\end{array}$ & $\begin{array}{c}\text { Crystalline } \\
\text { Phase } \\
\text { Separated }\end{array}$ \\
\hline MILSi-5 & 1150 & Crucible/In Crucible & Borosilicate & $X R D$ & Amorphous \\
\hline MiLSi-7 & 1400 & Crucible/In Crucible & Borosilicate & $\overline{\mathrm{XR}} \overline{\mathrm{D}}$ & Amorphous \\
\hline$\overline{\mathrm{MLSi}} \overline{8}$ & 1150 & Crucible//n Crucible & Borosilicate & $\mathrm{XRD}$ & Amorphous \\
\hline NILSi-9 & 1150 & Crucible//n Crucible & Borosilicate & $\overline{X R D}$ & Amorphous \\
\hline MLSi-11 & 1150 & Crucible/In Crucible & Borosilicate & XRD & Amorphous \\
\hline $\begin{array}{c}\mathrm{MIC4}- \\
144\end{array}$ & 1150 & Meîter/In Can & Borosilicate & $\overline{X R D}$ & Amorphous \\
\hline $\begin{array}{l}\text { IVIC } 4- \\
149 A\end{array}$ & 1150 & Nielter/ln Can & Borosilicate & XRD & $\mathrm{Li}_{3} \mathrm{PO}_{1}$ \\
\hline $\begin{array}{l}\text { MiC4 } \\
149 \mathrm{C}\end{array}$ & 1150 & Melter/ln Can & Borosilicate & XRD & $\overline{\mathrm{Li}} 3 \overline{\mathrm{PO}}_{4}$ \\
\hline $\begin{array}{l}\text { MIC5- } \\
9 A\end{array}$ & 1150 & Melter/In Can & Borosilicate & XRD & $\mathrm{Li}_{3} \mathrm{PO}_{4}$ \\
\hline $\begin{array}{l}\text { MIC5- } \\
\text { I3A }\end{array}$ & 1150 & Melter/ln Can & Borosilicate & $\overline{\mathrm{XRD}}$ & $\mathrm{Li}_{3} \overline{\mathrm{PO}}_{4}$ \\
\hline $\begin{array}{l}\text { MICS- } \\
21 A\end{array}$ & $11 \overline{50}$ & Melter/In Can & Borosilicate & $\overline{X R D}$ & $\begin{array}{c}\mathrm{Li}_{3} \overrightarrow{\mathrm{PO}}_{4}+ \\
\mathrm{SiO}_{2}\end{array}$ \\
\hline $\begin{array}{l}\text { MIC5- } \\
42 \mathrm{~A}\end{array}$ & 1150 & Meiter/ln Can & Borosilicate & $\overline{X R D}$ & $\mathrm{Li}_{3} \mathrm{PO}_{4}$ \\
\hline $\begin{array}{l}\text { MlC } 5- \\
42 \mathrm{C}\end{array}$ & $11 \overline{50}$ & Melter/ln Can & Borosilicate & XRD & $\overline{\mathrm{Li}_{3}} \overline{\mathrm{PO}_{4}}$ \\
\hline $\begin{array}{c}\text { COZZI } \\
12 X\end{array}$ & 1350 & Crucible/Patty & $\begin{array}{c}\mathrm{Na}_{2} \mathrm{O}-\mathrm{P}_{2} \mathrm{O}_{5} \\
\mathrm{~B}_{2} \mathrm{O}_{3}-\mathrm{SiO}_{2}\end{array}$ & TEM & $\mathrm{SiO}_{2}$ \\
\hline $\begin{array}{c}\text { COZZI } \\
12 \mathrm{X}-\mathrm{HT}\end{array}$ & 1350 & Crucible/Patty & $\begin{array}{c}\mathrm{Na}_{2} \mathrm{O}-\mathrm{P}_{2} \mathrm{O}_{5} \\
\mathrm{~B}_{2} \mathrm{O}_{3}-\mathrm{SiO}_{2}\end{array}$ & $\overline{\text { TEM }}$ & $\overline{\mathrm{SiO}_{2}}$ \\
\hline $\begin{array}{l}\text { COZZI } \\
12 X P 3\end{array}$ & 1350 & Crucible/Patty & $\begin{array}{c}\mathrm{Na}_{2} \mathrm{O}-\mathrm{P}_{2} \mathrm{O}_{5}^{-} \\
\mathrm{B}_{2} \mathrm{O}_{3}-\mathrm{SiO}_{2}\end{array}$ & TEM & Amorphous \\
\hline $\begin{array}{c}\text { COZZI } \\
\text { 12XP3- } \\
\text { HT }\end{array}$ & 1350 & Crucible/Patty & $\begin{array}{c}\mathrm{Na}_{2} \mathrm{O}-\mathrm{P}_{2} \mathrm{O}_{5}- \\
\mathrm{B}_{2} \mathrm{O}_{3}-\mathrm{SiO}_{2}\end{array}$ & TEM & Amorphous \\
\hline $\begin{array}{l}\text { COZZI } \\
12 X P 5\end{array}$ & 1300 & Crucible/Patty & $\begin{array}{c}\mathrm{Na}_{2} \mathrm{O}-\mathrm{P}_{2} \mathrm{O}_{5} \\
\mathrm{~B}_{2} \mathrm{O}_{3}-\mathrm{SiO}_{2}\end{array}$ & TEM & Amorphous \\
\hline $\begin{array}{c}\text { COZZI } \\
\text { 12XP5- } \\
\text { HT }\end{array}$ & 1300 & Crucible/Patty & $\begin{array}{c}\mathrm{Na}_{2} \mathrm{O}-\mathrm{P}_{2} \mathrm{O}_{5} \\
\mathrm{~B}_{2} \mathrm{O}_{3}-\mathrm{SiO}_{2}\end{array}$ & TEM & Amorphous \\
\hline PFP-1 & 1150 & Crucible/Patty & Borosilicate & XRD/SEM & Amorphous \\
\hline PFP-2 & 1150 & Crucible/Patty & Borosilicate & XRD/SEM & $\begin{array}{c}\mathrm{Li}_{3} \mathrm{PO}_{4}+ \\
\mathrm{Cr} 2 \mathrm{O}_{3}\end{array}$ \\
\hline PFP-3 & 1150 & Crucible/Patty & Borosilicate & XRD/SEM & $\begin{array}{c}\mathrm{Li}_{3} \mathrm{PO}_{4}+ \\
\mathrm{Cr} 2 \mathrm{O}_{3}\end{array}$ \\
\hline PFP-4 & 1150 & Crucible/Patty & Borosilicate & XRD/SEM & $\begin{array}{c}\mathrm{Li}_{3} \mathrm{PO}_{4}+ \\
\mathrm{Cr}_{2} \mathrm{O}_{3}\end{array}$ \\
\hline
\end{tabular}




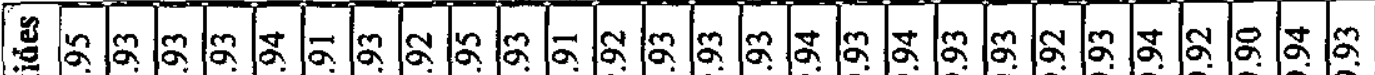

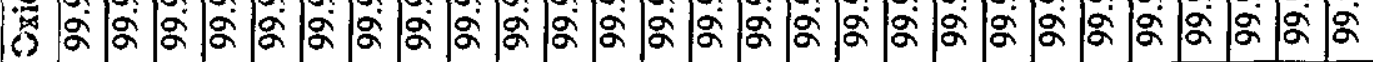

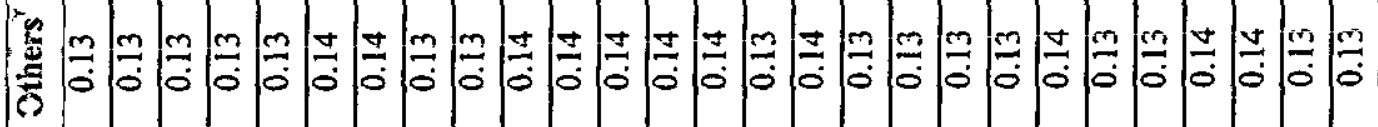
心 L

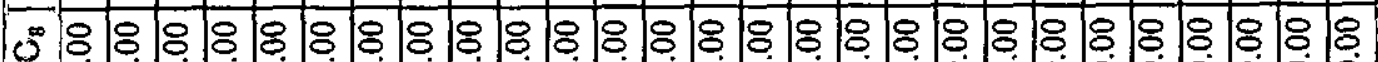

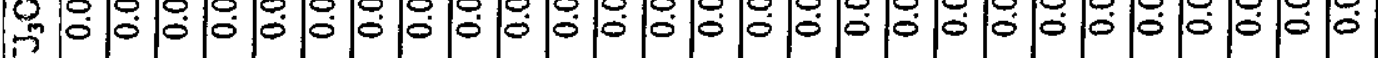

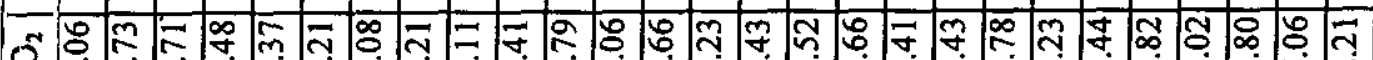

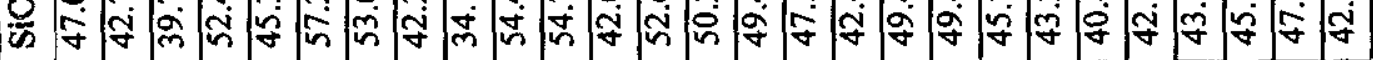
m 긍ㅇㅇ응

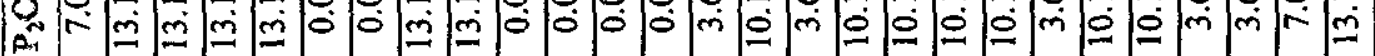

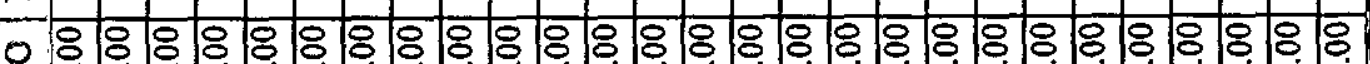

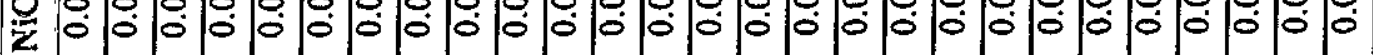
그유

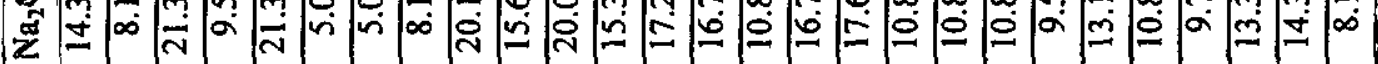

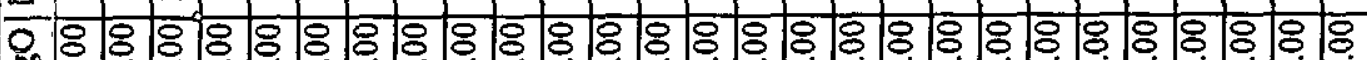

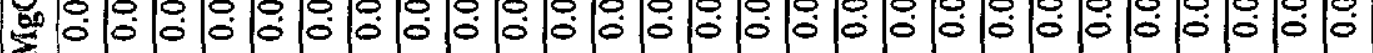
O

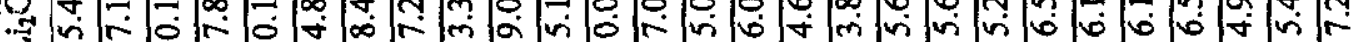

a

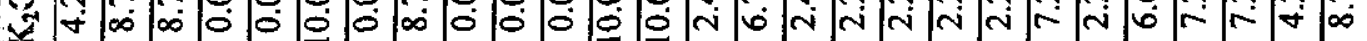

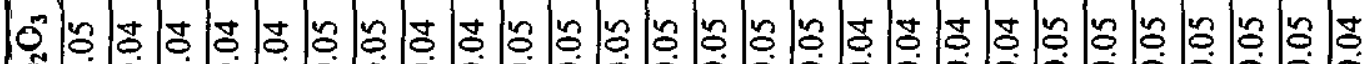

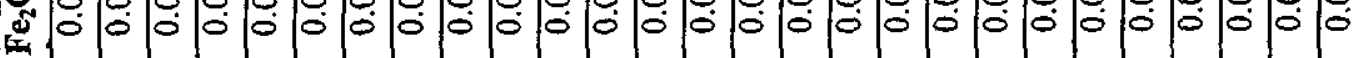
10 -

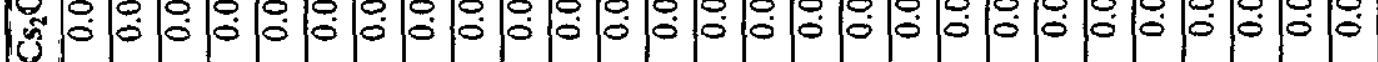
○

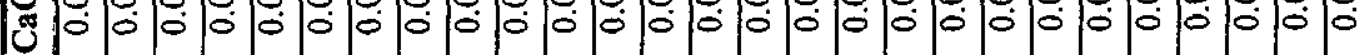

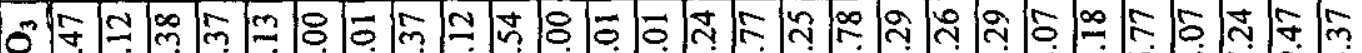
pa ón

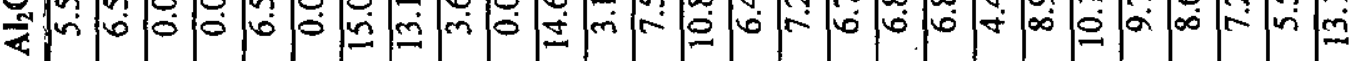

을

흘

西 它 


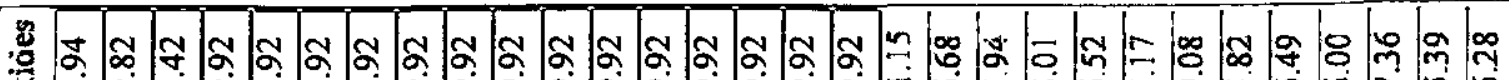

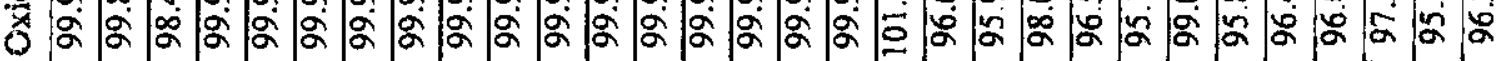

m (5)

\% A

-

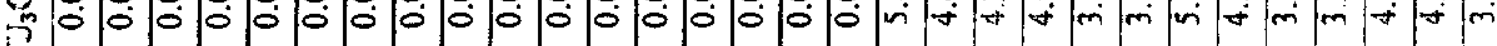

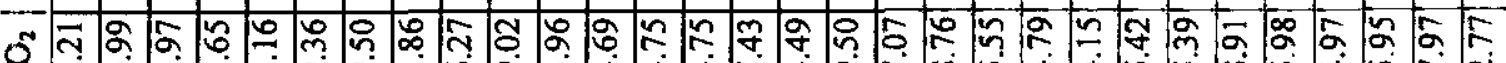

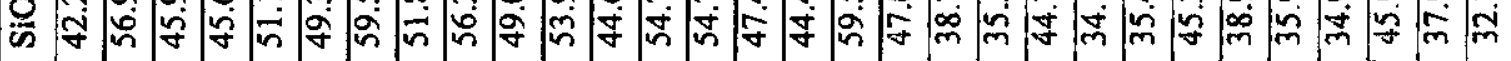
চ in

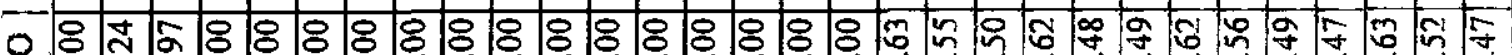

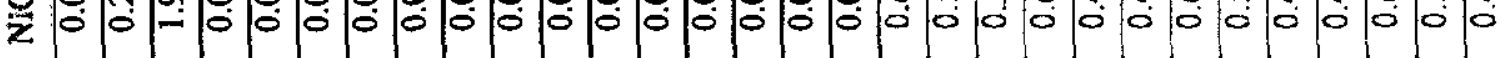

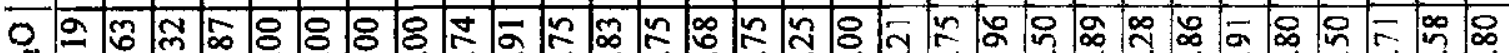

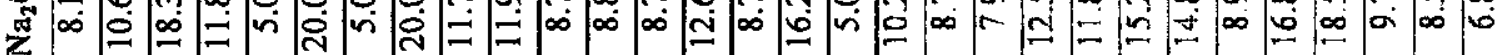

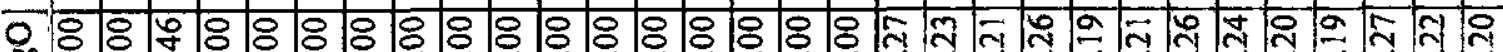

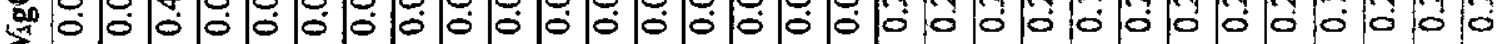

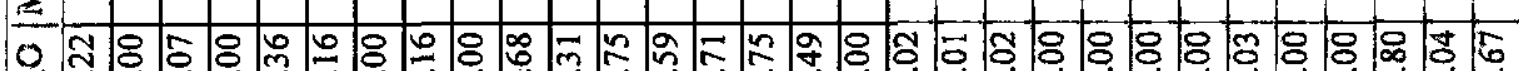

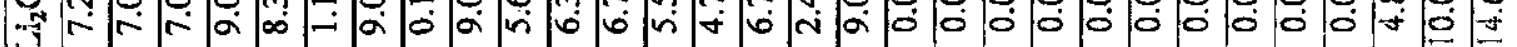

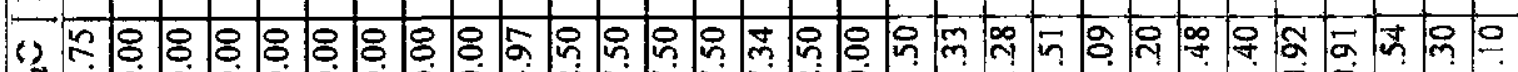

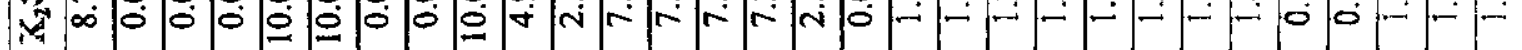

б

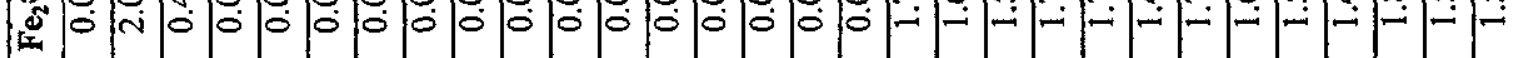

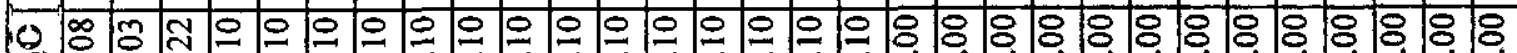

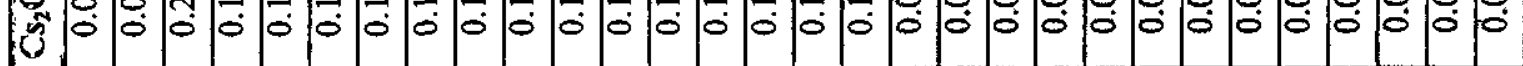

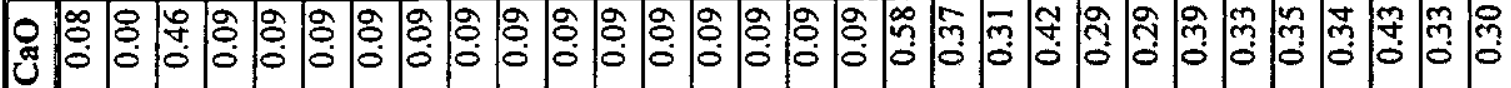
o

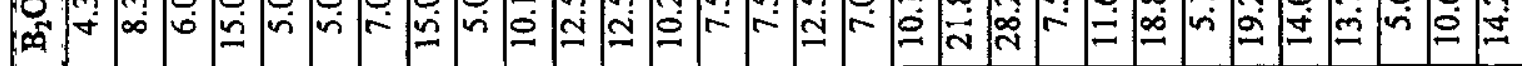

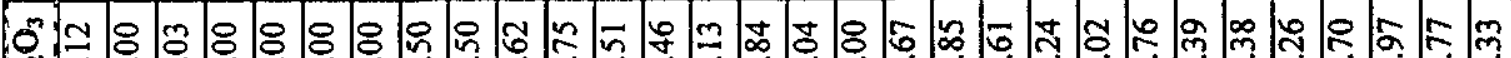

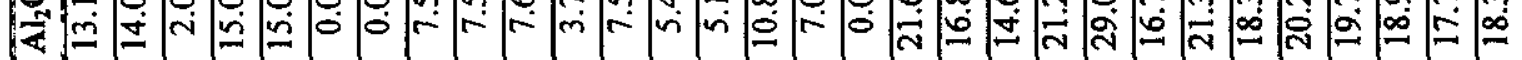

园

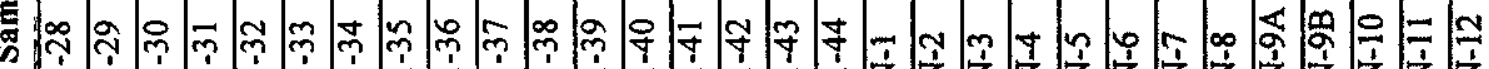

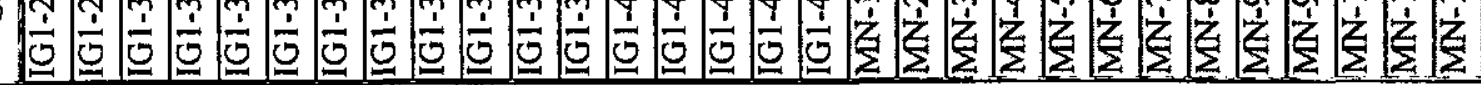




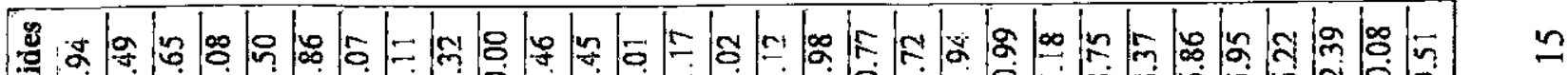

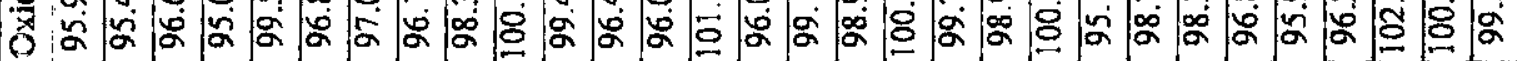

"๘̆ : 0 0

๘

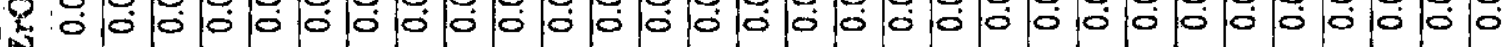

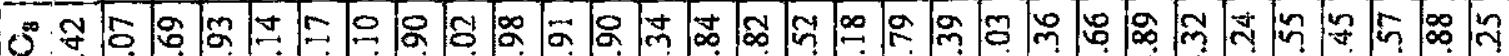

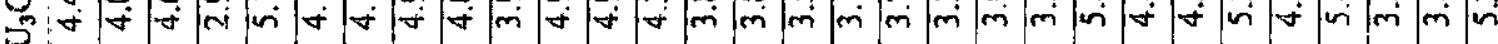

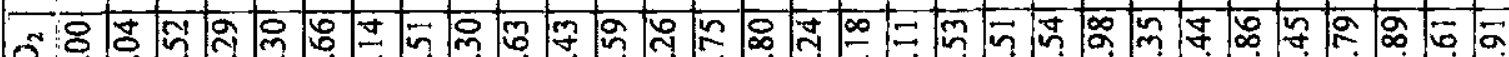

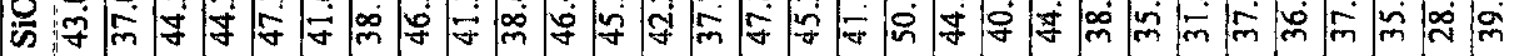
о유

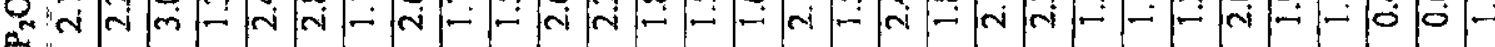

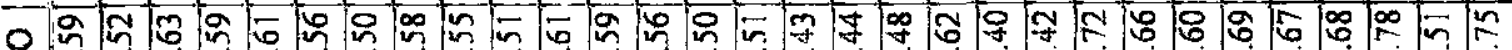

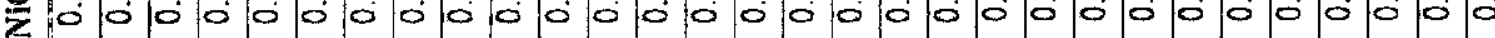

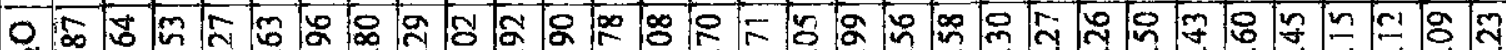
ż山E

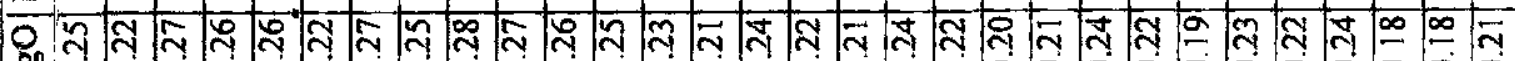

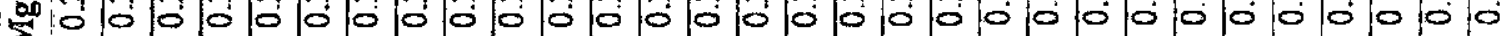

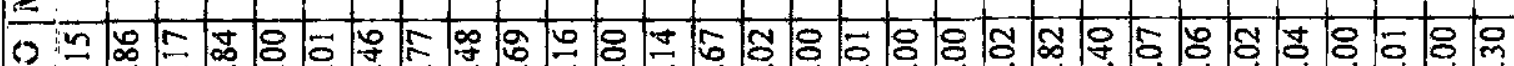

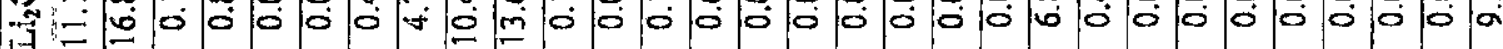

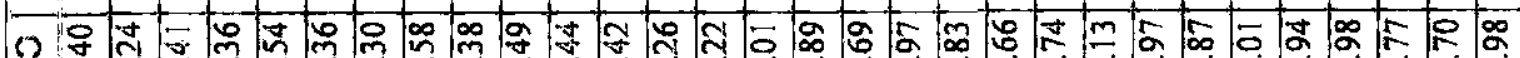

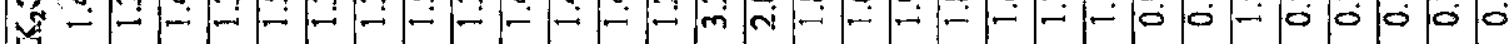
రై

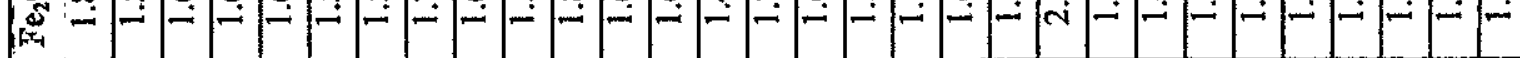

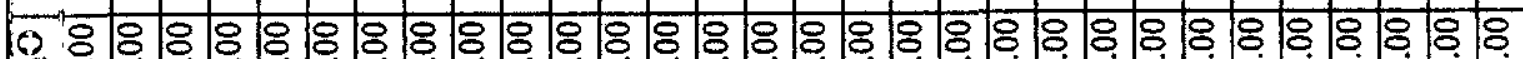

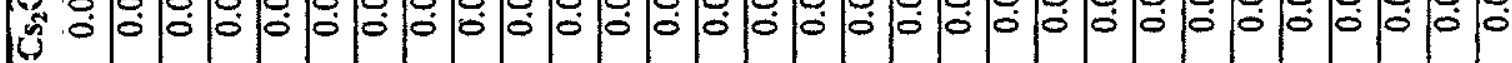
0 ․․ U

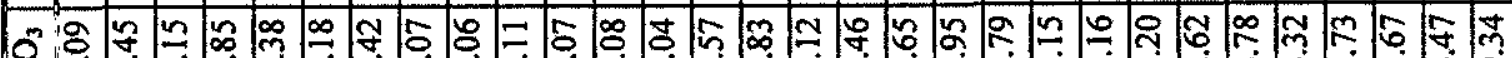

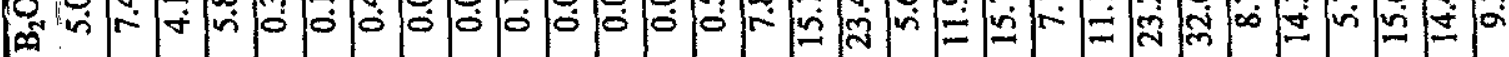

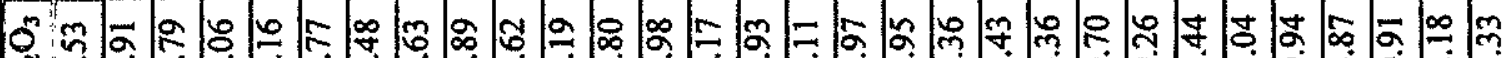

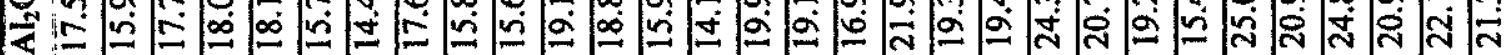

일

을

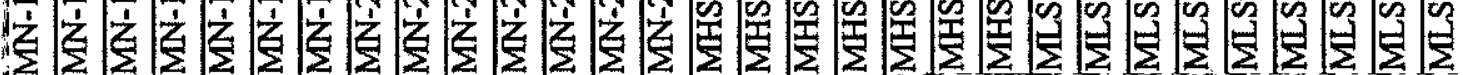




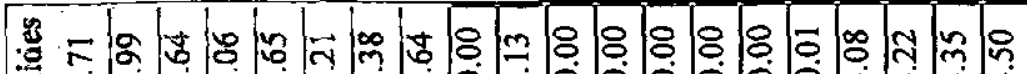

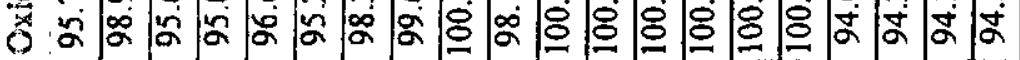

㤎 象

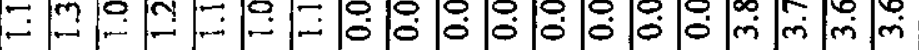

"\%

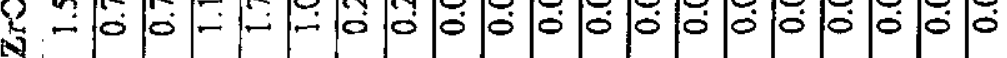

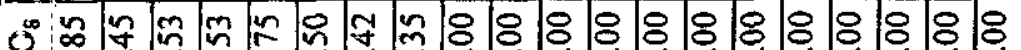

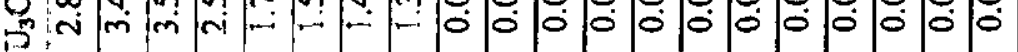

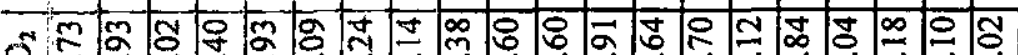

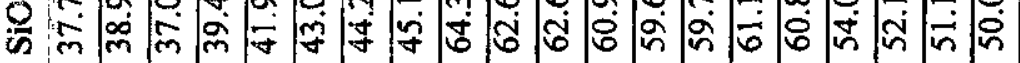

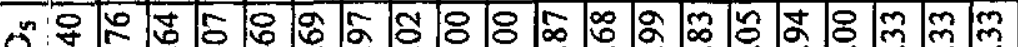

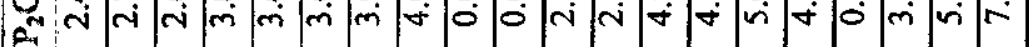

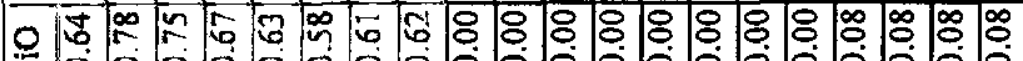
z

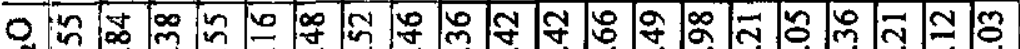
تِ O

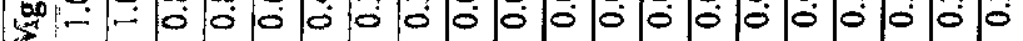

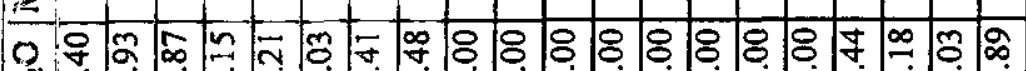
$\because$ m

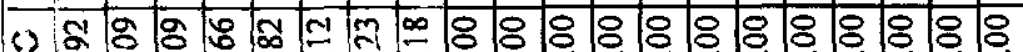
r.

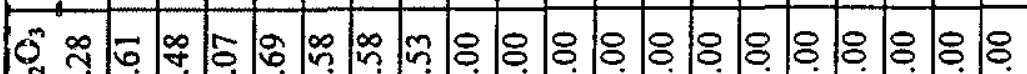

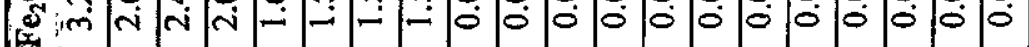

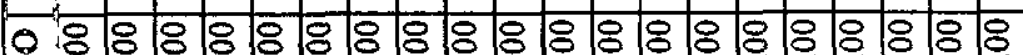

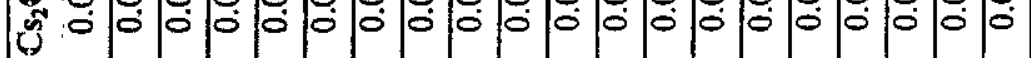

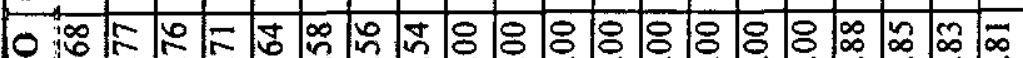

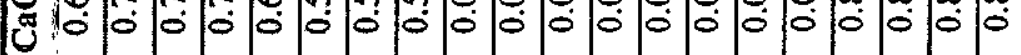
0m

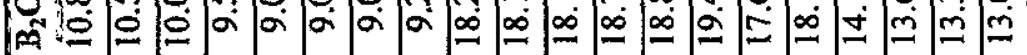

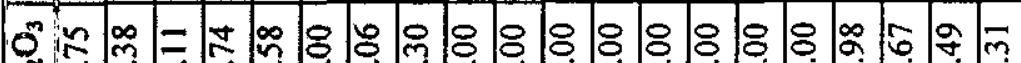

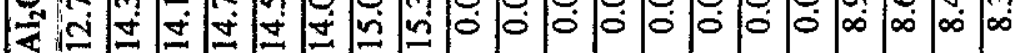

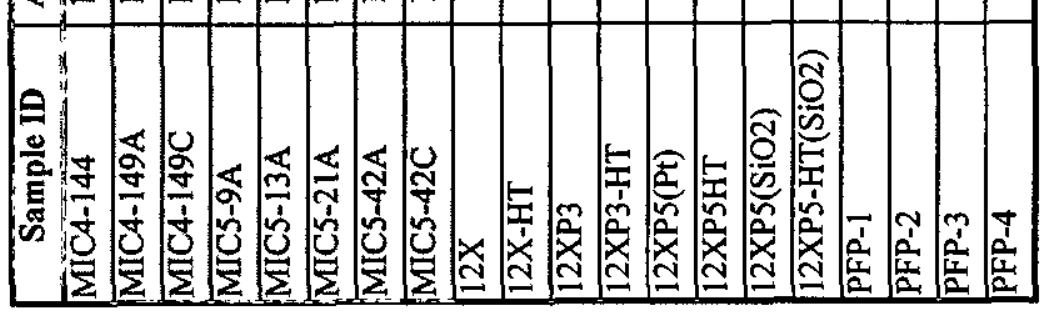


Table $\mathbb{I V}$. Database of $\mathbb{B}$ orosilicate $\mathbb{G l a s s e s}$ Contaiming $\mathbb{P}_{2} \mathbb{O}_{5}: \mathbb{D} u r a b i l i t y ~ R e s p o n s e$

\begin{tabular}{|c|c|c|c|c|c|c|c|c|}
\hline Sampie & $\begin{array}{c}\text { Mean } \\
\text { Weasured } \\
\text { pHz }\end{array}$ & 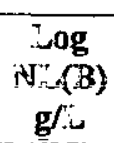 & $\begin{array}{c}\mathrm{j} / \mathrm{g} \\
\mathrm{g} / \mathrm{L} \\
\mathrm{L}\end{array}$ & 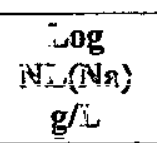 & $\begin{array}{c}\log \\
i \mathrm{v}(\mathrm{Si}) \\
\mathrm{g} /:\end{array}$ & 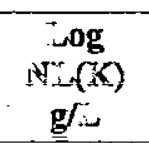 & 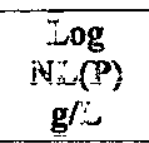 & $\begin{array}{l}\text { [SBF } \\
\text { WWA] }\end{array}$ \\
\hline IG1-01 & 11.48 & 0.74 & 0.515318 & 0.658742 & 0.127089 & -1.54752 & 0.50845 & 7.53 \\
\hline IG1-02 & 9.96 & 0.85 & 0.243913 & 0.776491 & -0.07283 & -1.86064 & 0.24768 & $-8.2 \overline{8}$ \\
\hline IGI-03 & 11.43 & 1.63 & 1.083531 & 1.518615 & 0.710851 & -1.86114 & 1.62967 & 139.85 \\
\hline IG1-04 & 10.78 & -0.12 & -0.01555 & 0.078302 & -0.3982 & -- & 0.07902 & 4.32 \\
\hline IG1-05 & 10.07 & 0.91 & -0.59135 & 0.650671 & -0.09579 & $\cdots$ & 0.42773 & -5.93 \\
\hline IG1-06 & 10.79 & 1.28 & 1.280384 & 1.252325 & 0.500573 & -1.91913 & -- & -22.66 \\
\hline IG1-07 & 10.2 & -0.25 & -0.14206 & -0.93903 & -0.28843 & $\cdots$ & -- & -2.71 \\
\hline IG1-08 & 10.83 & -0.45 & -0.24717 & -0.1687 & -0.59751 & -1.86114 & -0.16091 & 2.31 \\
\hline IG1-09 & 10.23 & 0.76 & 0.347087 & 0.661228 & -0.30202 & --- & 0.50132 & $10 . \overline{40}$ \\
\hline IG1-10 & 12.45 & 1.47 & 1.328698 & 1.27923 & 1.051953 & -- & $-\cdots$ & 67.01 \\
\hline IG1-11 & 11.94 & -0.24 & -0.11968 & 0.272815 & -0.24746 & $\ldots$ & -- & 8.70 \\
\hline IG1-12 & 11.04 & 0.96 & - & 0.903713 & -0.50802 & -1.91956 & -- & -2.30 \\
\hline IG1-13 & 12.7 & 1.77 & 1.667325 & 1.768626 & 1.60366 & -1.91956 & $-\cdots$ & 109.17 \\
\hline IG1-14 & 11.55 & 0 & -0.24509 & 0.114519 & -0.40989 & -1.30295 & -0.63774 & 3.64 \\
\hline IG1-15 & 10.99 & -0.01 & -0.10184 & 0.067145 & -0.39112 & -1.74972 & -0.07802 & 2.01 \\
\hline IGI-16 & 11.64 & -0.15 & -0.36524 & 0.075279 & -0.5041 & -1.30295 & -0.37620 & $\overline{3.82}$ \\
\hline IG1-17 & 11.08 & -0.39 & -0.88381 & -0.17413 & -0.62517 & -1.27324 & -0.51905 & 1.68 \\
\hline IG1-18 & 10.11 & -0.2 & -0.24145 & -0.15177 & -0.56695 & -1.27324 & -0.20364 & 0.36 \\
\hline IG1-19 & 10.06 & -0.25 & -0.2998 & -0.18975 & -0.60305 & -1.27324 & -0.23601 & 0.30 \\
\hline IG1-20 & 10.15 & -0.3 & -0.35723 & -0.24914 & -0.65939 & -1.27324 & -0.29858 & 0.22 \\
\hline IG1-21 & 11.49 & 0.25 & 0.168103 & 0.169491 & -0.58576 & -1.77887 & -0.37781 & 2.94 \\
\hline IG1-22 & 10.5 & -0.1 & -0.35185 & -0.10173 & -0.58924 & -1.27324 & -0.29583 & 0.91 \\
\hline IG1-23 & 10.91 & -0.46 & -0.38333 & -0.18298 & -0.64871 & -1.74064 & -0.26646 & 1.71 \\
\hline IG1-24 & 11.49 & 0.32 & 0.159823 & 0.176573 & -0.57528 & -1.77887 & -0.27498 & 1.89 \\
\hline IG1-25 & 11.7 & -0.05 & -0.09545 & 0.101429 & -0.53897 & -1.77887 & -0.37422 & 4.01 \\
\hline IGl-26 & 11.5 & 0.79 & 0.559598 & 0.697732 & 0.157479 & -1.54752 & 0.55159 & 8.10 \\
\hline IG1-27 & 10.94 & -0.52 & -0.23141 & -0.17156 & -0.62679 & -1.86114 & -0.17369 & 2.58 \\
\hline IG1-28 & 10.97 & -0.45 & -0.20205 & -0.14698 & -0.58723 & -1.86114 & -0.11722 & 2.66 \\
\hline IG 1-29 & 10.83 & -0.35 & -0.14701 & -0.45739 & -0.38751 & -- & 0.43410 & -0.44 \\
\hline IG1-30 & 12.59 & 1.39 & 1.212621 & 1.254079 & 0.836097 & -- & 0.79169 & 88.57 \\
\hline IGI-31 & 11.14 & 0.48 & 0.405468 & 0.195434 & -0.27628 & --- & -- & 4.27 \\
\hline IG1-32 & 10.37 & -0.41 & -0.2389 & -0.2708 & -0.52274 & -1.91913 & -0.19564 & 0.98 \\
\hline IG1-33 & 12.49 & 1.07 & 1.286663 & 1.327485 & 0.933076 & -1.91913 & -- & 65.06 \\
\hline IG1-34 & 9.85 & 0.03 & 0.034918 & -0.62393 & -0.28921 & -.. & -0.03816 & -0.36 \\
\hline IG1-35 & 10.8 & -0.06 & 1.565891 & -0.14688 & -0.51824 & $\cdots$ & -0.73028 & 2.15 \\
\hline IG1-36 & 12.15 & 1.25 & 1.254658 & 1.252287 & 0.967117 & -1.91913 & $\cdots$ & 63.76 \\
\hline IG1-37 & 9.9 & 1.19 & -0.97893 & 1.104823 & -0.01456 & -1.61549 & 0.87843 & -3.97 \\
\hline IG1-38 & 10.12 & 0.39 & 0.345961 & 0.168987 & -0.20103 & -1.31707 & 0.36654 & -0.90 \\
\hline IG1-39 & 11.12 & 0.53 & 0.446287 & 0.334414 & -0.46938 & -1.79419 & -0.39468 & 4.08 \\
\hline IG1-40 & 10.7 & 0.92 & 0.754279 & 0.860677 & 0.332697 & -1.79419 & 0.51839 & -2.06 \\
\hline IG1-41 & 11.73 & 1.61 & 1.21406 & 1.526705 & 1.102907 & -1.79419 & 0.73005 & -13.30 \\
\hline IG1-42 & 10.45 & -0.4 & -0.27761 & -0.21255 & -0.5985 & -1.78483 & -0.37739 & 1.26 \\
\hline
\end{tabular}




\begin{tabular}{|c|c|c|c|c|c|c|c|c|}
\hline Sample & $\begin{array}{c}\text { Wiegn } \\
\text { Wieasureci } \\
\text { pr: }\end{array}$ & $\begin{array}{c}\text { Jog } \\
N \cdot(B) \\
\text { g/t }\end{array}$ & $\begin{array}{l}\text { iog } \\
\text { Nu(i) } \\
\text { g/t }\end{array}$ & $\begin{array}{c}\text { iog } \\
N i(\mathbf{N} \mathbf{a}) \\
\mathrm{g} / \mathrm{L}\end{array}$ & $\begin{array}{c}\text { تuog } \\
\mathrm{Nic(Si)} \\
\mathrm{g} / \mathrm{L}\end{array}$ & $\begin{array}{c}\operatorname{sog} \\
\operatorname{Nin}(\mathrm{k}) \\
\mathrm{g} / \mathrm{L}\end{array}$ & $\begin{array}{c}\operatorname{bog} \\
N i\left(R^{2}\right) \\
g / L\end{array}$ & $\begin{array}{l}{[S B \mid-} \\
|\forall \dot{A}|\end{array}$ \\
\hline IG1-43 & 10.22 & 0.16 & -0.14965 & 0.043111 & -0.6216 & -1.31707 & -0.50380 & 0.00 \\
\hline IG1-44 & 9.77 & 0.06 & 0.058648 & -0.60078 & -0.26072 & & -0.02201 & -0.47 \\
\hline iVN-1 & 8.66 & -0.69 & -0.30961 & -0.56534 & -0.76691 & -0.99422 & $\cdots$ & -1.00 \\
\hline VIN-2 & 7.81 & $t .15$ & $0 . \overline{849125}$ & 0.522302 & 0.70253 & -0.47756 & - & $-\overline{80} . \overline{29}$ \\
\hline$\overline{\mathrm{MN}}-3$ & 6.78 & $1 . \overline{78}$ & 1.05955 & 1.12568 & $-\overline{0} . \overline{485} \overline{2}$ & 0.165006 & $=$ & -454.51 \\
\hline$\overline{\mathrm{MN}-4}$ & 8.36 & -0.43 & $-0 . \overline{88939}$ & -0.44422 & -0.60151 & .0 .99553 & - & -1.19 \\
\hline MIN-5 & 8.74 & -0.46 & -1.32307 & -0.38111 & -0.62232 & -0.77963 & - & -0.90 \\
\hline $\sin -6$ & 9.03 & 1.14 & 0.98585 & 0.879653 & -0.84576 & 0.131023 & - & -37.44 \\
\hline MN-7 & 9.32 & -0.48 & -1.32307 & -0.35467 & -0.55969 & -0.89837 & - & -0.41 \\
\hline MN-8 & 8.11 & 1.03 & 0.15176 & 0.520234 & $-0 . \overline{73354}$ & -0.45835 & $-\infty$ & -50.93 \\
\hline $\mathrm{MN}-\overline{9} \mathrm{~A}$ & 9.8 & $0 . \overline{8}$ & -0.31236 & 0.729461 & -0.69021 & 0.170715 & $\cdots$ & 2.31 \\
\hline WN-9B & $\overline{9.8}$ & $0 . \overline{82}$ & -0.01133 & 0.687644 & -0.67784 & $0.17189 \overline{9}$ & $-\cdots$ & 2.31 \\
\hline $\mathrm{MN}-1 \overline{0}$ & 11.51 & -1.37 & -3.34837 & 0.462679 & 0.45617 & -0.16505 & - & 6.57 \\
\hline MN-11 & 11.17 & 0.15 & 0.121775 & 0.01512 & 0.17538 & -0.56218 & - & 3.32 \\
\hline$\overline{\mathrm{MN}}-\overline{12}$ & $1 \overline{8} 86$ & $0 . \overline{77}$ & 0.735957 & 0.571601 & 0.175261 & $-0.0 \overline{7936}$ & $\rightarrow$ & 29.54 \\
\hline$\overline{M N}-\overline{1}$ & 11.54 & 0.09 & 0.105139 & $0.078 \overline{68}$ & -0.12825 & -0.3927 & $\ldots$ & 5.55 \\
\hline MN-14 & 11.94 & 0.67 & 0.67561 & 0.586283 & 0.30378 & -0.08125 & $\cdots$ & 40.80 \\
\hline MIN-15 & 10.32 & -0.34 & -0.9276 & $-0.12 \overline{268}$ & -0.48184 & -0.81852 & $=$ & 1.34 \\
\hline MN-16 & 10.02 & 0.01 & -0.46765 & 0.029393 & -0.53597 & $-\overline{0} 6 \overline{2092}$ & - & 2.37 \\
\hline $\mathrm{MN}-17$ & 10.09 & -0.87 & -1.32307 & $-0 . \overline{36391}$ & -0.78617 & 0.9106 & - & 0.92 \\
\hline MNN-18 & 10.72 & -0.16 & -1.58836 & 0.081112 & -0.65754 & -0.52322 & $\cdots$ & 5.51 \\
\hline$M N-19$ & 11.02 & -0.14 & 0.01444 & $0 . \overline{276949}$ & -0.52413 & -0.31102 & - & 11.17 \\
\hline พลN-20 & 10.12 & -1.24 & $-0.3 \overline{3634}$ & -0.38129 & -0.64488 & -0.68868 & -- & $1.0 \overline{3}$ \\
\hline$i \mathrm{iN}-21$ & 10.94 & $-0 . \overline{3}$ & 0.157696 & 0.080102 & -0.49056 & -0.37286 & - & 11.48 \\
\hline MiN-22 & 11.08 & $-0.1 \overline{2}$ & 0.512185 & 0.353971 & -0.11955 & -0.19847 & $\cdots$ & 30.87 \\
\hline MN-23 & 10.01 & -1.32 & -1.22652 & -0.65418 & -1.08527 & -1.01961 & -- & 0.11 \\
\hline $\mathrm{MN}-24$ & 10.33 & -0.71 & -1.32307 & -0.11955 & -0.67341 & -0.8145 & $-\cdots$ & 2.30 \\
\hline MN-25 & 10.82 & -0.68 & -0.54716 & 0.331962 & -0.25069 & -0.17227 & $\cdots$ & 10.86 \\
\hline MN-26 & 11.34 & -0.36 & 0.727859 & 0.937818 & 0.077372 & 0.187891 & --- & 70.53 \\
\hline MHSi-1 & 8.39 & -0.71 & -2.16817 & -0.55493 & -0.74475 & -1.11864 & - & -0.96 \\
\hline MHSi-2 & 8.5 & -0.31 & -1.19042 & -0.42067 & -0.70769 & -1.25575 & -- & -2.47 \\
\hline MHSi-3 & 7.76 & 1.32 & 0.426891 & 0.65859 & -0.70732 & -0.37584 & $\cdots$ & -131.04 \\
\hline MHSi-4 & 8.53 & -0.63 & -1.6241 & -0.492 & -0.69336 & -1.10204 & -- & -0.84 \\
\hline MHSi-5 & 8.7 & -0.33 & -1.32307 & -0.34181 & -0.62632 & -0.90568 & $\cdots$ & -1.30 \\
\hline MHSi-6 & 8.73 & 0.31 & -2.27731 & 0.111424 & -0.60407 & -0.70689 & $-\cdots$ & -5.31 \\
\hline MHSi-11 & 10.38 & -0.29 & -0.20008 & -0.53098 & $-0 . \overline{4063}$ & -0.55481 & -- & -0.29 \\
\hline MLSi-1 & 8.59 & -0.5 & -0.16356 & -0.48597 & -0.60112 & -0.75816 & $\cdots$ & -1.11 \\
\hline MLSi-2 & 8.39 & 1.08 & 0.863417 & 0.607622 & -0.76291 & -0.21374 & $\cdots$ & -66.60 \\
\hline MLSi-3 & 7.02 & 1.87 & 1.613212 & 1.421663 & -0.4686 & 0.658102 & 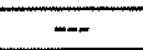 & -611.10 \\
\hline MLSi-4 & 8.69 & -0.35 & -2.32307 & -0.31139 & -0.55764 & -0.6396 & $-\cdots$ & -0.67 \\
\hline MLSi-5 & 9.01 & 0.48 & 0.031388 & 0.303884 & -0.6166 & -0.3513 & - & -5.09 \\
\hline MLSi-7 & 9.33 & -0.14 & -1.6241 & -0.11845 & -0.47182 & -0.48871 & $-\cdots$ & 0.96 \\
\hline MLSi-8 & 9.31 & 1.06 & -0.57311 & 0.633218 & -0.83616 & 0.217571 & $-\cdots$ & -21.42 \\
\hline MLSi-9 & 10.2 & 0.95 & -1.32307 & 0.725601 & -0.78503 & 0.391596 & $\cdots$ & 9.36 \\
\hline
\end{tabular}


WSRC-TR-2000-00339

\begin{tabular}{|c|c|c|c|c|c|c|c|c|}
\hline $\begin{array}{l}\text { Sampie } \\
T D\end{array}$ & $\begin{array}{c}\text { Wiean } \\
\text { Weasured } \\
\text { pH }\end{array}$ & $\begin{array}{c}\log \\
N L(B) \\
g / L\end{array}$ & $\begin{array}{c}J 0 g \\
\text { NLC(i) } \\
\text { g/L }\end{array}$ & $\begin{array}{c}\text { Log } \\
\text { NL(Na) } \\
\mathrm{g} / \mathrm{l}\end{array}$ & $\begin{array}{c}\mathrm{j} 0 \mathrm{~g} \\
\mathrm{NL}(\mathbf{S i}) \\
\mathrm{g} / \mathrm{L}\end{array}$ & $\begin{array}{c}\text { Log } \\
\text { NL(K) } \\
\mathrm{g} / \mathrm{L}\end{array}$ & $\begin{array}{c}\log \\
N L(P) \\
\mathrm{g} / L\end{array}$ & $\begin{array}{l}{[S B]-} \\
{[W A]}\end{array}$ \\
\hline MLSSi-11 & 1128 & 0.41 & 0.290433 & 0.248985 & -0.2155 & -0.32523 & $\cdots$ & 0.62 \\
\hline $\begin{array}{c}\text { MIC4- } \\
144\end{array}$ & 11 & -0.26 & -0.29297 & -0.16549 & -0.49502 & -0.76619 & $\cdots$ & 1.12 \\
\hline $\begin{array}{l}\text { MIC4- } \\
149 \mathrm{~A}\end{array}$ & 11.1 & -0.16 & -0.27128 & -0.08464 & .0 .46316 & -0.71548 & $\cdots$ & 161 \\
\hline $\begin{array}{l}\text { MIC4- } \\
149 \mathrm{C}\end{array}$ & 11.3 & -0.17 & -0.24202 & -0.08837 & 0.45006 & -0.70437 & $\ldots$ & 1.73 \\
\hline $\begin{array}{l}\text { MIC5 } \\
9 \mathrm{~A}\end{array}$ & 10.87 & -0.39 & -0.20485 & -0.22466 & -0.53899 & -0.73438 & $\cdots$ & 1.38 \\
\hline $\begin{array}{c}\text { MПC5 } \\
13 \mathrm{~A}\end{array}$ & 10.99 & .0 .45 & 0.21592 & -0.3094 & -0.57575 & -0.71864 & -- & 1.10 \\
\hline $\begin{array}{c}\text { MIC5 } \\
21 \mathrm{~A}\end{array}$ & 10.76 & -0.45 & -0.12532 & -0.28877 & -0.55346 & -0.71768 & $\cdots$ & 1.25 \\
\hline $\begin{array}{c}\text { MiC5 } \\
42 \mathrm{~A}\end{array}$ & 10.67 & -0.43 & -0.11933 & 0.29911 & -0.521 & -0.6867 & $\cdots$ & 1.34 \\
\hline $\begin{array}{l}\text { MIC5 } \\
42 \mathrm{C}\end{array}$ & 10.75 & -0.43 & -0.10988 & -0.3012 & -0.52616 & -0.67401 & $\cdots$ & 1.38 \\
\hline $\begin{array}{c}\text { COZZI } \\
12 \mathrm{X}\end{array}$ & 10.35 & -0.67 & $\cdots$ & -1.02986 & -2.12848 & $\cdots$ & -- & -0.67 \\
\hline $\begin{array}{r}\mathrm{COZZZI} \\
12 \mathrm{X}-\mathrm{HT}\end{array}$ & 10.37 & -0.6 & $\cdots$ & -0.97136 & -2.2063 & $\cdots$ & $\cdots$ & -0.77 \\
\hline $\begin{array}{l}\text { COZZI } \\
12 X P 3\end{array}$ & 10.35 & -0.28 & $\cdots$ & -0.92568 & -1.9363 & -- & $-\cdots$ & -2.22 \\
\hline $\begin{array}{c}\text { COZZI } \\
12 \mathrm{XP3}- \\
\mathrm{HT}\end{array}$ & 10.03 & -0.25 & $\cdots$ & -0.9473 & -2.08442 & -- & $\cdots$ & -2.50 \\
\hline $\begin{array}{l}\text { COZZI } \\
12 X P 5\end{array}$ & no data & no data & $\cdots$ & no data & no data & $\cdots$ & $\cdots$ & no data \\
\hline $\begin{array}{l}\text { COZZI } \\
12 X P 5 \\
\text { HT }\end{array}$ & no data & no data & -- & no data & no data & $\cdots$ & $\cdots$ & no data \\
\hline $\begin{array}{l}\text { COZZI } \\
12 X P 5\end{array}$ & 9.74 & -0.3 & $\cdots$ & -0.96009 & -2.32591 & -- & $\cdots$ & -2.02 \\
\hline $\begin{array}{c}\text { COZZI } \\
12 X P S- \\
\mathrm{HT}\end{array}$ & 9.71 & -0.29 & $-\cdots$ & -0.90578 & -2.23392 & -- & -- & -2.08 \\
\hline PFP-1 & no data & no data & no data & no data & no data & no data & no data & no data \\
\hline PFP-2 & no data & no data & no data & no data & no data & no data & no data & no data \\
\hline PFP-3 & no data & no data & no data & no data & no data & no data & no data & no data \\
\hline PFP:4 & no data & no data & no data & no data & no data & no data & no data & no data \\
\hline
\end{tabular}


Table $V$. Borosilicate Glasses Comtaining $\mathbb{L}_{2} \bigcirc$ and $\mathbb{E} x$ hibibing $\mathbb{C P S}$

\begin{tabular}{|c|c|c|c|c|c|c|c|}
\hline $\begin{array}{l}\mathrm{P}_{2} \mathrm{O}_{5} \\
(\mathrm{w} \% \%)\end{array}$ & $\begin{array}{l}\mathrm{B}_{2} \mathrm{O}_{3} \\
(\mathrm{wt} \%)\end{array}$ & {$\left[\begin{array}{l}\Sigma \mathrm{IM}_{2} \mathrm{O} \\
(\mathrm{wt} \%)^{*}\end{array}\right.$} & $\begin{array}{l}\mathrm{SiO}_{2} / \\
\mathrm{B}_{2} \mathrm{O}_{3}\end{array}$ & $\begin{array}{l}\text { Major } \\
\text { Alkali }\end{array}$ & CPS Phase & $\begin{array}{l}\text { Glass + } \\
\text { Wieit . } \\
\text { Temp }\left({ }^{\circ}\right)\end{array}$ & Ref. \\
\hline $\begin{array}{c}1.94- \\
4.0\end{array}$ & $\begin{array}{l}5.68- \\
10.65\end{array}$ & $\begin{array}{l}15.83- \\
25.74\end{array}$ & $\begin{array}{l}3.56- \\
8.09\end{array}$ & $\begin{array}{c}\mathrm{Na}_{2} \mathrm{O}+ \\
\mathrm{Li}_{2} \mathrm{O}\end{array}$ & $\begin{array}{c}8 \text { with } \\
\mathrm{Li}_{3} \mathrm{PO}_{4} \\
+3 \text { with } \\
\mathrm{SiO}_{2} \text { or } \\
\text { Silicate } \\
\end{array}$ & $\begin{array}{c}11 \text { M-Area } \\
\text { MLLW } \\
\text { Glasses } \\
\text { Various } \\
\text { Temps }\end{array}$ & $\begin{array}{l}\text { This } \\
\text { study }\end{array}$ \\
\hline $\begin{array}{l}3.62- \\
13.12\end{array}$ & $\begin{array}{l}4.37- \\
13.13\end{array}$ & $\begin{array}{l}14.10- \\
25.59\end{array}$ & $\begin{array}{l}2.60- \\
12.00\end{array}$ & $\begin{array}{c}\mathrm{Li}_{2} \mathrm{O}+ \\
\mathrm{Na}_{2} \mathrm{O}\end{array}$ & $\begin{array}{c}24 \text { with } \\
\mathrm{Li}_{3} \mathrm{PO}_{4} \text { and } \\
\mathrm{Li}_{2} \mathrm{NaPO}_{4}\end{array}$ & $\begin{array}{c}24 \text { Idaho } \\
\text { HAW } \\
\text { Glasses@ } \\
1150^{\circ} \mathrm{C}\end{array}$ & $\begin{array}{c}\text { This } \\
\text { study; } \\
\text { Staples, } \\
\text { et. al } \\
(1998)^{\text {Err }} \\
\text { or! Bookmark } \\
\text { not defined. }\end{array}$ \\
\hline
\end{tabular}

\subsection{CRYSTALIINE PHASE SEPARATION IN MIXED WASTIE GLASSES: PILOT SCALE TESTING}

A large amount of data on actual mixed waste glasses containing 1-5 wt $\% \mathrm{U}_{3} \mathrm{O}_{8}$ was generated by SRTC while performing a mixed (hazardous and radioactive) waste treatability study which included a pilot scale melter demonstration. In particular, several tanks of this mixed waste in the Savannah River Site (SRS) M-Area contained high phosphate, while several tanks were almost phosphate free. A pilot scale melter run was performed under funding from the Mixed Waste Focus Area (EM-50).

The melter campaign was initiated with low phosphate tank waste. Subsequently, high phosphate containing waste was fed to the melter. The amount of phosphate in the final glass was analyzed chemically and the glasses were analyzed by XRD. The glasses made at the beginning of the campaign had 2.40 wt\% $\mathrm{P}_{2} \mathrm{O}_{5}$ before the high $\mathrm{P}_{2} \mathrm{O}_{5}$ tank waste was fed to the melter. The $2.4 \mathrm{wt} \% \mathrm{P}_{2} \mathrm{O}_{5}$ glass exhibited no crystalline phases at the beginning of the melter run (Figure 6a). As the campaign progressed, glasses containing $\geq 2.76 \mathrm{wt} \% \mathrm{P}_{2} \mathrm{O}_{5}$ began to crystallize $\mathrm{Li}_{3} \mathrm{PO}_{4}$ upon cooling indicating that the crystalline phase separation begins at $\sim 2.6 \mathrm{wt} \% \mathrm{P}_{2} \mathrm{O}_{5}$ (see Table I and Figure 6a horizontal line) even when the $\mathrm{Al}_{2} \mathrm{O}_{3}$ content reached $\sim 15 \mathrm{wt} \%$ (Figure $6 \mathrm{~b}$ ). This indicates that high $\mathrm{Al}_{2} \mathrm{O}_{3}$ does not suppress CPS in these phosphate containing glasses as it does in borosilicate systems that undergo APS. ${ }^{13}$ 


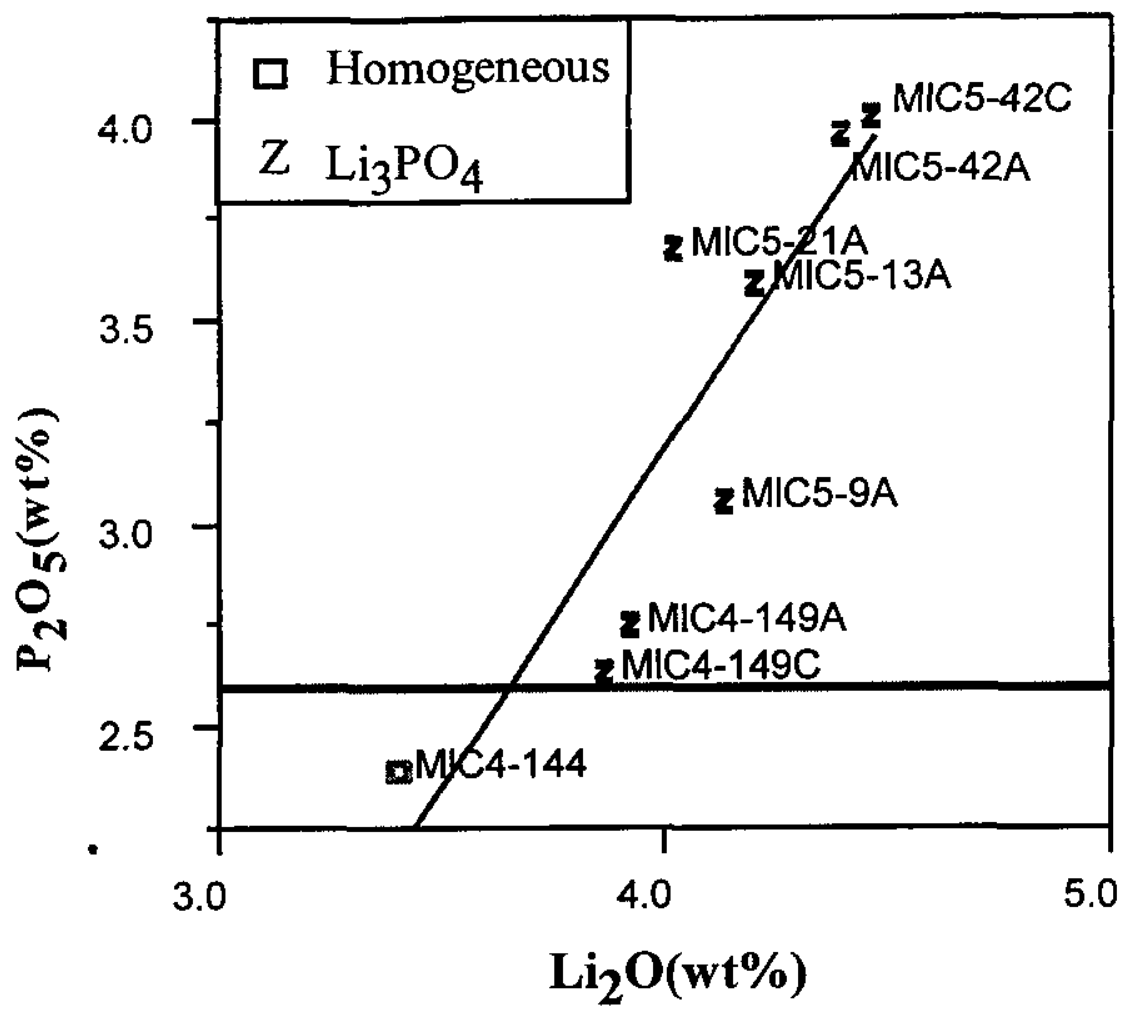

Figure 6a. Phosphate glass crystalline phase separation (CPS) began in glasses produced during a pilot scale melter runs of a mixed waste as a high $\mathrm{P}_{2} \mathrm{O}_{5}$ containing feed was fed to a melter of homogeneous glass. CPS started at about $2.6 \mathrm{wt} \%$ $\mathrm{P}_{2} \mathrm{O}_{5}$ the horizontal line indicated on the figure. 
WSRC-TR-2000-00339

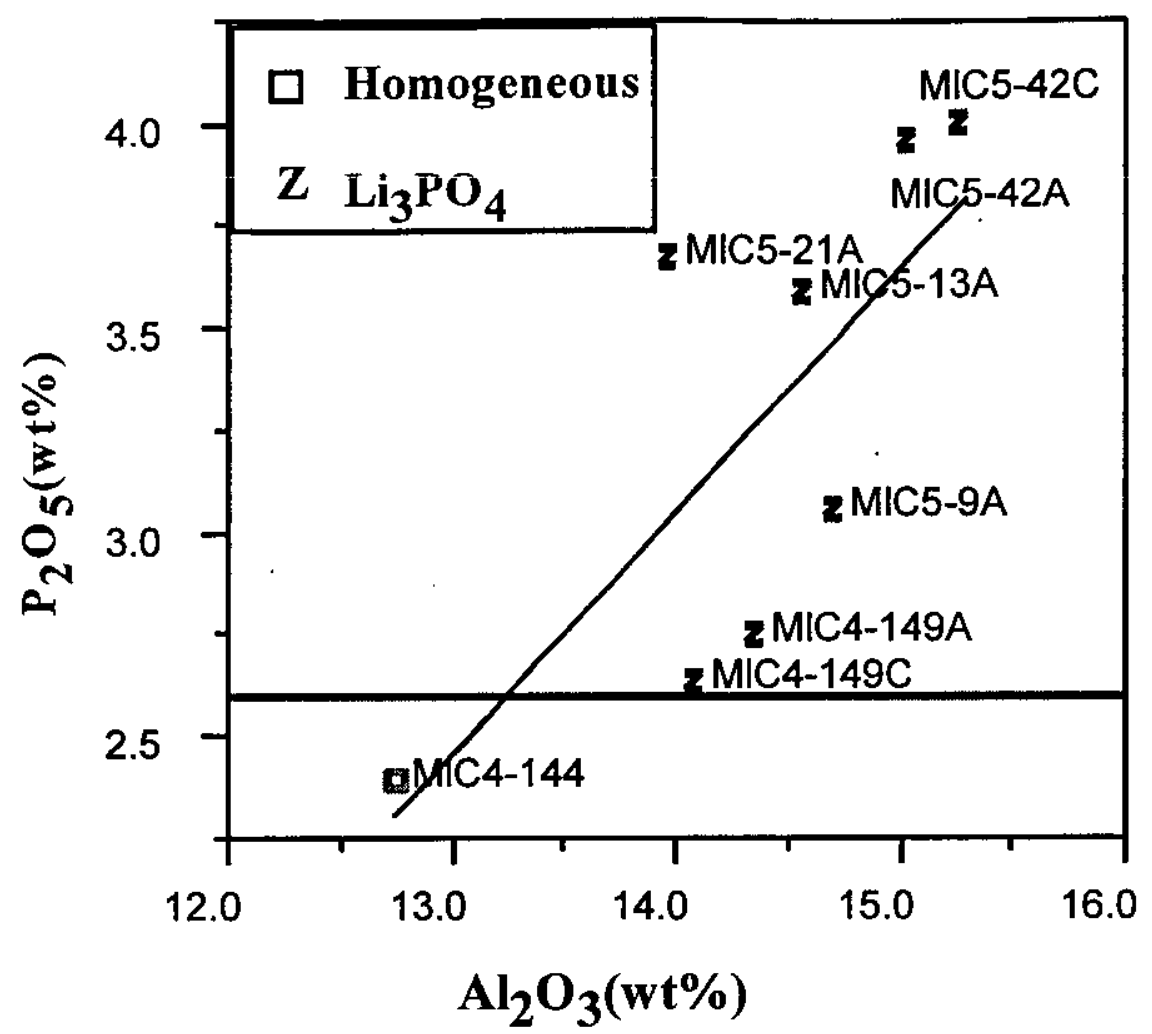

Figure $6 \mathrm{~b}$. Alumina contents as high as $15 \mathrm{wt} \%$ did not stabilize the glass against the CPS as it does in borosilicate glasses undergoing APS. 


\subsection{CRYSTALLINE PHASE SEPARATION IN BOROSILICATE GLASSES: CRUBIBLE AND PILOT SCALE TESTING}

Analysis of the chemistry of the 83 crucible study glasses from the M-Area, INEEL, and the Cozzi studies were combined with the 8 glasses from the M-Area melter run. The combined data ( 91 glasses) supported the data from the melter campaign: glasses with greater than $\sim 2.6 \mathrm{wt} \% \mathrm{P}_{2} \mathrm{O}_{5}$ underwent some type of CPS (see Table II and Figure 7). Only eight glasses were reported to be homogeneous at $>2.6 \mathrm{wt} \% \mathrm{P}_{2} \mathrm{O}_{5}$ : four of the simple glasses from the Cozzi study (shown in the ellipses in Figure 7a and 7b), three INEEL glasses (IG1-14, -35, -43), and one M-Area glass (MN-15). Conversley, several glasses were reported to have CPS ( $\mathrm{Z}, \mathrm{X}$, and $\mathrm{Y}$ on Figure 7$)$ at $<2.6 \mathrm{wt} \% \mathrm{P}_{2} \mathrm{O}_{5}$. These glasses were two of the Cozzi study simple glasses that crystallized only $\mathrm{SiO}_{2}(12 \mathrm{X}$ and 12X-HT indicated by a $Y$ in Figures $7 \mathrm{a}$ and $7 \mathrm{~b}$ ), one INEEL glass (IG1-30) that exceeded its solubility limit for $\mathrm{ZrO}_{2}$, and two M-Area glasses (MN-5 and MHSi-11) which exceeded the solubility limit for $\mathrm{Al}_{2} \mathrm{O}_{3}$ (one crystallized $\alpha-\mathrm{Al}_{2} \mathrm{O}_{3}$ while the other crystallized $\mathrm{LiAlO}_{2}$ ).

The data shown in Figure 7 indicates that the Cozzi study simple glasses are poor representations of the complexity of the CPS in complex waste glasses. This may be attributed to the fact that the Cozzi study glasses contained only one alkali $\left(\mathrm{Na}_{2} \mathrm{O}\right)$ while the INEEL and M-Area waste glasses contain complex combinations of alkalis and alkaline earth oxides. For the discussion of the effects of $\mathrm{P}_{2} \mathrm{O}_{5}$ type CPS on glass durability (Section 6.0), the 6 simple glasses, the IG1-30 glass, the MN-5, and the MHSi11 glasses will be omitted from the database. 


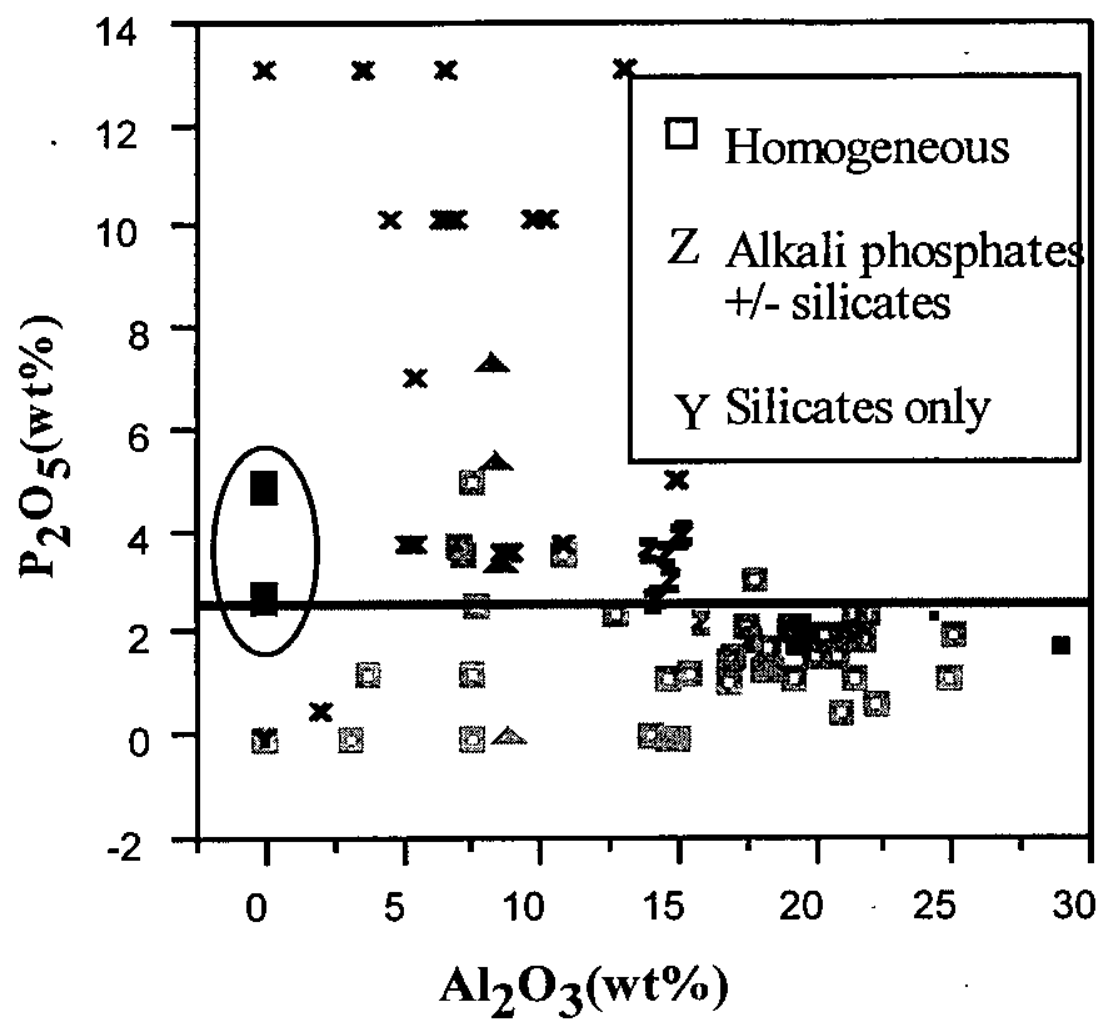

Figure 7a.Data from 91 borosilicate waste glasses containing varying amounts of $\mathrm{P}_{2} \mathrm{O}_{5}$ plotted against the amount of $\mathrm{Al}_{2} \mathrm{O}_{3}$ in each glass indicates that complex waste glasses with $\geq 2.6 \mathrm{wt} \% \mathrm{P}_{2} \mathrm{O}_{5}$ (horizontal line) undergo crystalline phase separation of $\mathrm{P}_{2} \mathrm{O}_{5}$-rich and/or $\mathrm{SiO}_{2}$-rich phases. The glasses in the ellipse are the simple four component glasses from Cozzi." 


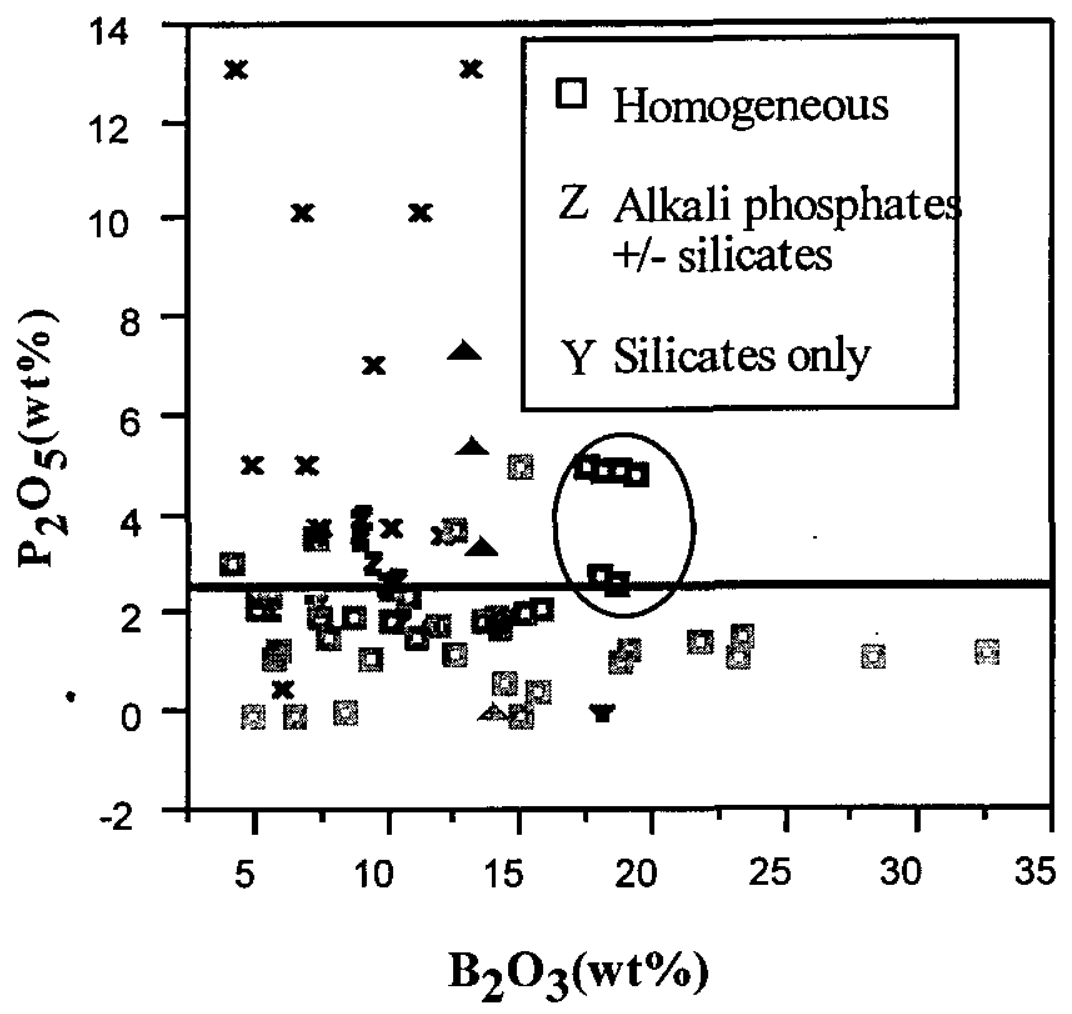

Figure 7a.Data from 91 borosilicate waste glasses containing varying amounts of $\mathrm{P}_{2} \mathrm{O}_{5}$ plotted against the amount of $\mathrm{B}_{2} \mathrm{O}_{3}$ in each glass indicates that complex waste glasses with $\geq 2.6 \mathrm{wt} \% \mathrm{P}_{2} \mathrm{O}_{5}$ (horizontal line) undergo crystalline phase separation of $\mathrm{P}_{2} \mathrm{O}_{5}$-rich and/or $\mathrm{SiO}_{2}$-rich phases. The glasses in the ellipse are the simple four component glasses from Cozzi. ${ }^{11}$ 


\subsection{EFFECTS OF CRYSTALLINE PHASE SEPARATION ON GLASS DURABILITY}

The relative durability of the 82 remaining $\mathrm{P}_{2} \mathrm{O}_{5}$ containing glasses in the database were examined as a function of their measured durability during PCT (ASTM C1285-97) testing. Normalized boron and sodium were chosen as the durability indicators because the slope relating these two parameters was $\sim 1$ (Figure 8) and the intercept was $\sim 0$ for the entire population of 82 glasses $\left(R^{2}=0.76\right)$. This indicated that the $B$ and $N a$ were not involved in the CPS and could be used as a good indicator of the overall glass durability.

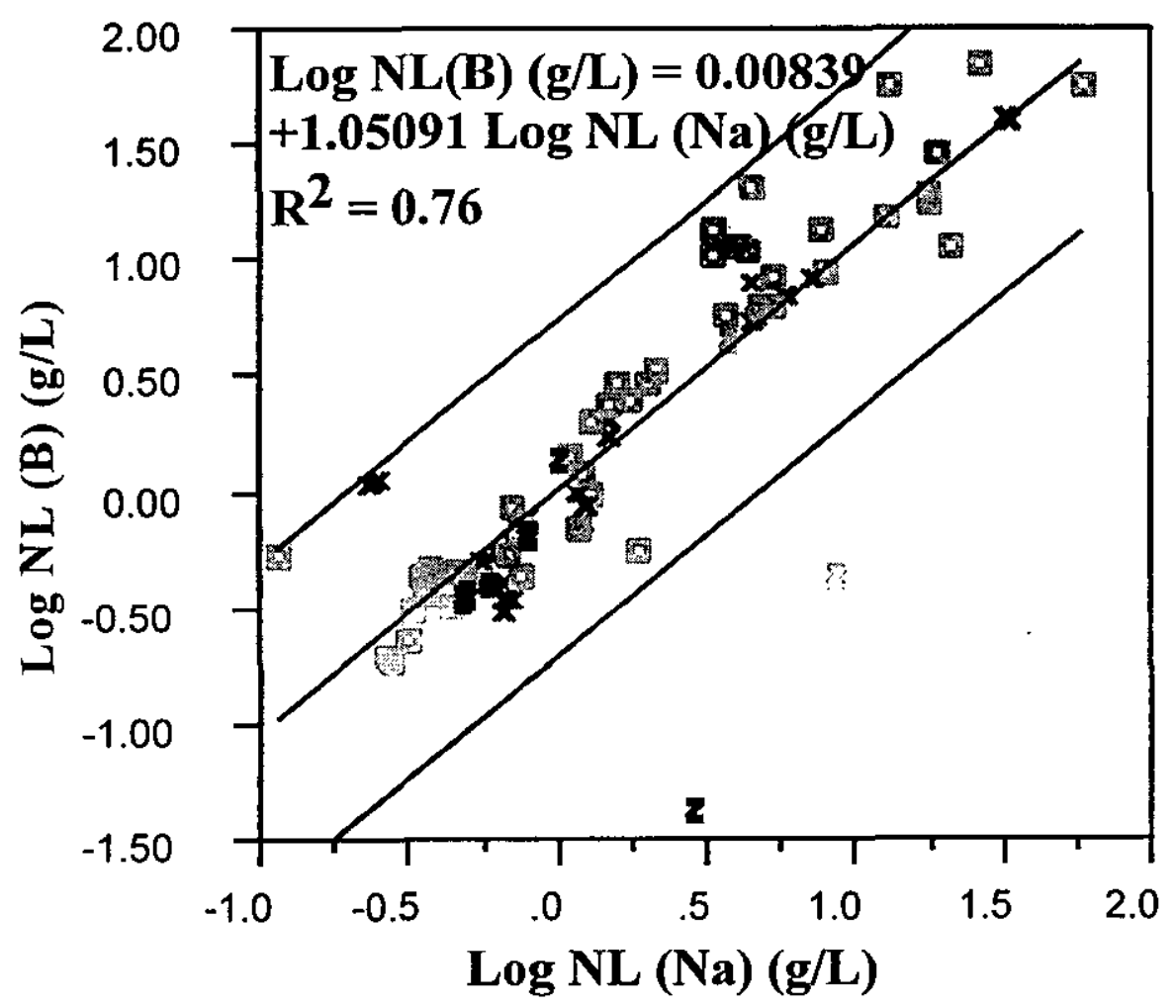

Figure 8. Plot of $\log \mathrm{NL}(\mathrm{B})$ vs. $\log \mathrm{NL}(\mathrm{Na})$ for 82 amorphous (indicated by squares) and CPS glasses (indicated by symbols $x$ and $z$ ) showing that the leaching of the $\mathrm{Na}$ and $\mathrm{B}$ is congruent (slope of $\sim 1$ and intercept of $\sim 0$ ). 
Conversely, a plot of the normalized release of boron verses the normalized release of lithium for the 44 glasses classified as homogeneous (visually and/or by $x$-ray analysis) gave a slope of less than unity, a non-zero intercept, and a very poor fit $\left(\mathrm{R}^{2}=0.44\right)$ indicating that $\mathrm{Li}$ was not a good indicator of overall glass durability (Figure $9 \mathrm{a}$ ) for the homogeneous glasses or that some of the glasses classified as "homogeneous" were, indeed, phase separated (APS). The release of B and Li from the 37 glasses that had undergone CPS gave a slope of unity, a near zero intercept, and an $R^{2}$ of 0.73 (Figure $9 b$ ). Figure $9 \mathrm{~b}$ indicates that $\mathrm{Li}$ is a good indicator of CPS. It also demonstrates that the normalized B release is somewhat greater than the Li release for the CPS glasses (perhaps since some Li was tied up in crystalline phases), making B a worst case indicator of the glass durability in the presence or absence of CPS.

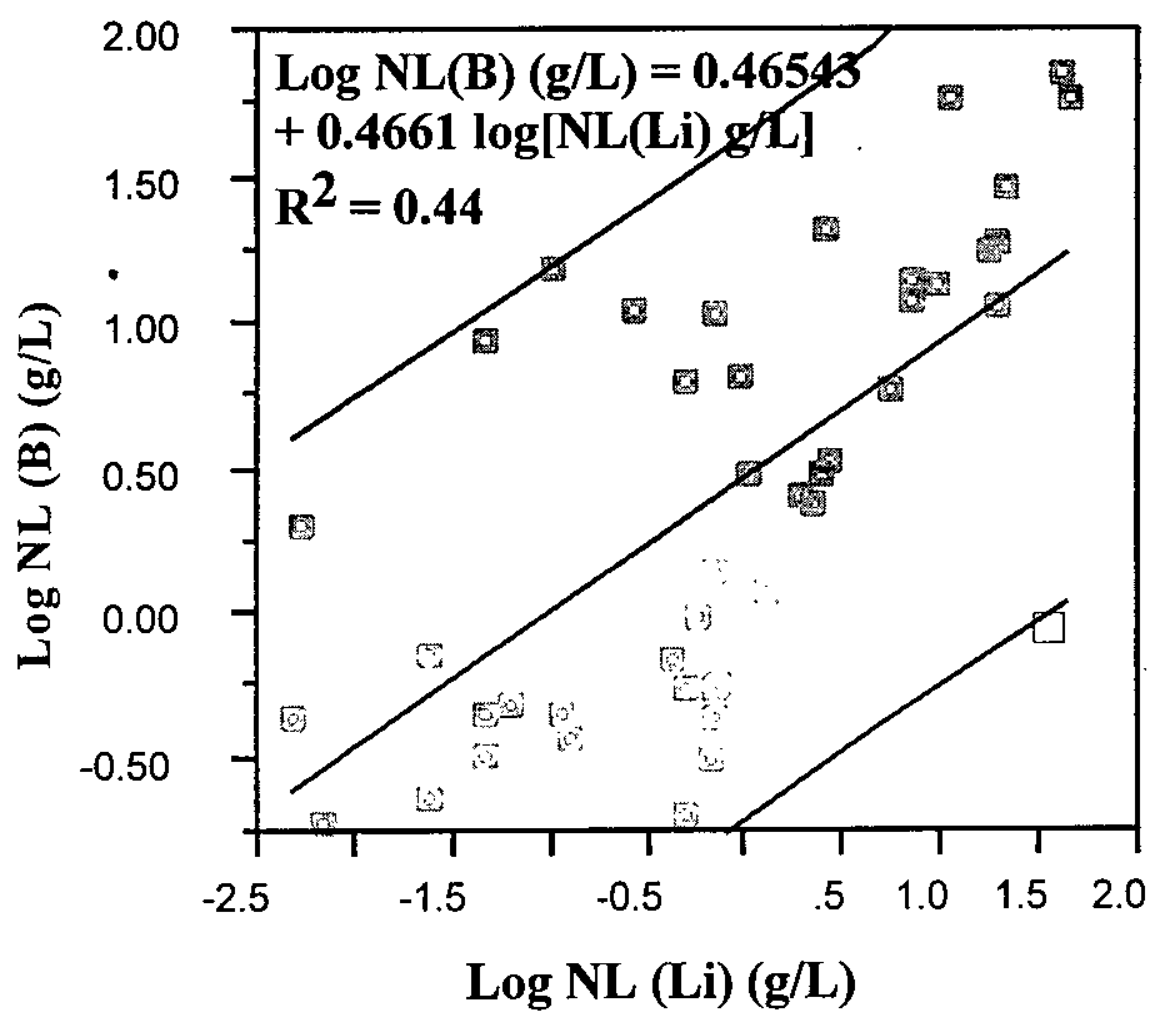

Figure 9a. Release of normalized $\mathrm{B}$ vs. Li for 50 homogeneous $\mathrm{P}_{2} \mathrm{O}_{5}$ glasses. 


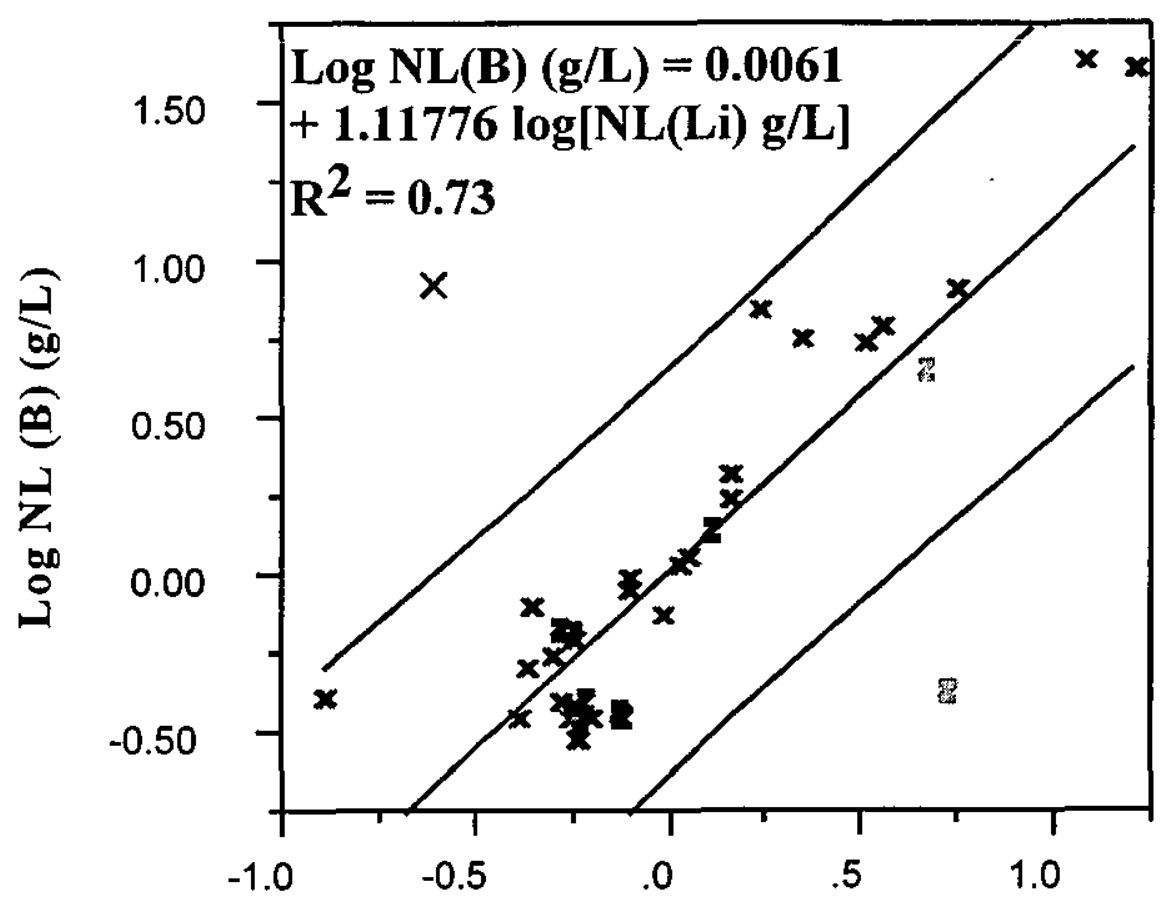

$\log N \mathbf{L}(\mathrm{Li})(\mathrm{g} / \mathrm{L})$

Figure 9b. Release of normalized B vs. Li for 37 crystallized CPS glasses.

Phosphate durability was available for the 44 glasses in the INEEL glass data set. There was no correlation between the $\log \mathrm{NL}(\mathrm{P})$ in $\mathrm{g} / \mathrm{L}$ and $\log \mathrm{NL}(\mathrm{Li})$ in $\mathrm{g} / \mathrm{L}$ for the homogeneous glasses. However, for the 24 of the 25 glasses undergoing CPS (one did not contain $\mathrm{Li}_{2} \mathrm{O}$ as a component) there is a strong correlation between the normalized $\mathrm{P}$ and $\mathrm{Li}$ release (Figure 10). Therefore, normalized $\mathrm{Li}$ release can be used as andicator of durability of the CPS phase (Figure $9 \mathrm{~b}$ and 10) while normalized $\mathrm{Na}$ and $\mathrm{B}$ release can be used as an indicator of the overall glass durability (Figure 8) 


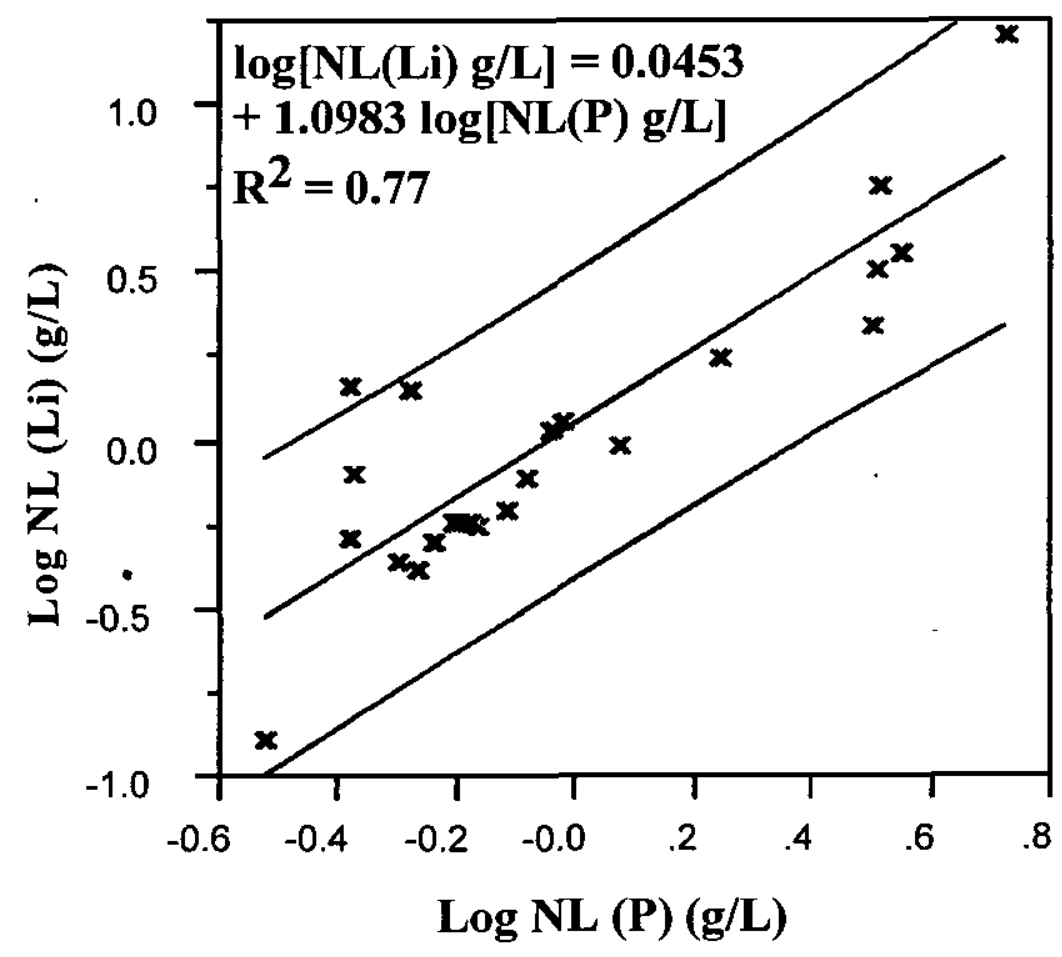

Figure 10. Release of normalized Li vs. P for 24 crystallized INEEL CPS glasses.

The durability of $82 \mathrm{P}_{2} \mathrm{O}_{5}$ containing glasses was assessed against the $\mathrm{P}_{2} \mathrm{O}_{5} /\left(\mathrm{B}_{2} \mathrm{O}_{3}+\right.$ $\mathrm{SiO}_{2}$ ) molar ratio of the glasses (Figure 11). The distribution of the glass populations were thought to be the following:

- 32 had undergone CPS of a phosphate phase identified by XRD

- 50 visually homogeneous or homogeneous by XRD

It was recognized that many of the glasses that were classified as "homogeneous" visually or by $\mathrm{XRD}$, may have undergone amorphous phase separation. The durability 
response shown in Figure 11 indicates that many of the high $\mathrm{P}_{2} \mathrm{O}_{5}$ containing CPS glasses were more durable than the low $\mathrm{P}_{2} \mathrm{O}_{5}$ containing homogeneous glasses circled in the figure. Many glasses classified as homogeneous were poorly durable and performed at or above EA glass limit in terms of their normalized B release (Figure 11a). Only a few of the high $\mathrm{P}_{2} \mathrm{O}_{5}$ containing CPS glasses were poorly durable and performed at or above EA glass limit (Figure 1 1a). The CPS does not appear to be the cause of the adverse effects on the waste glass durability (see discussion in next section).

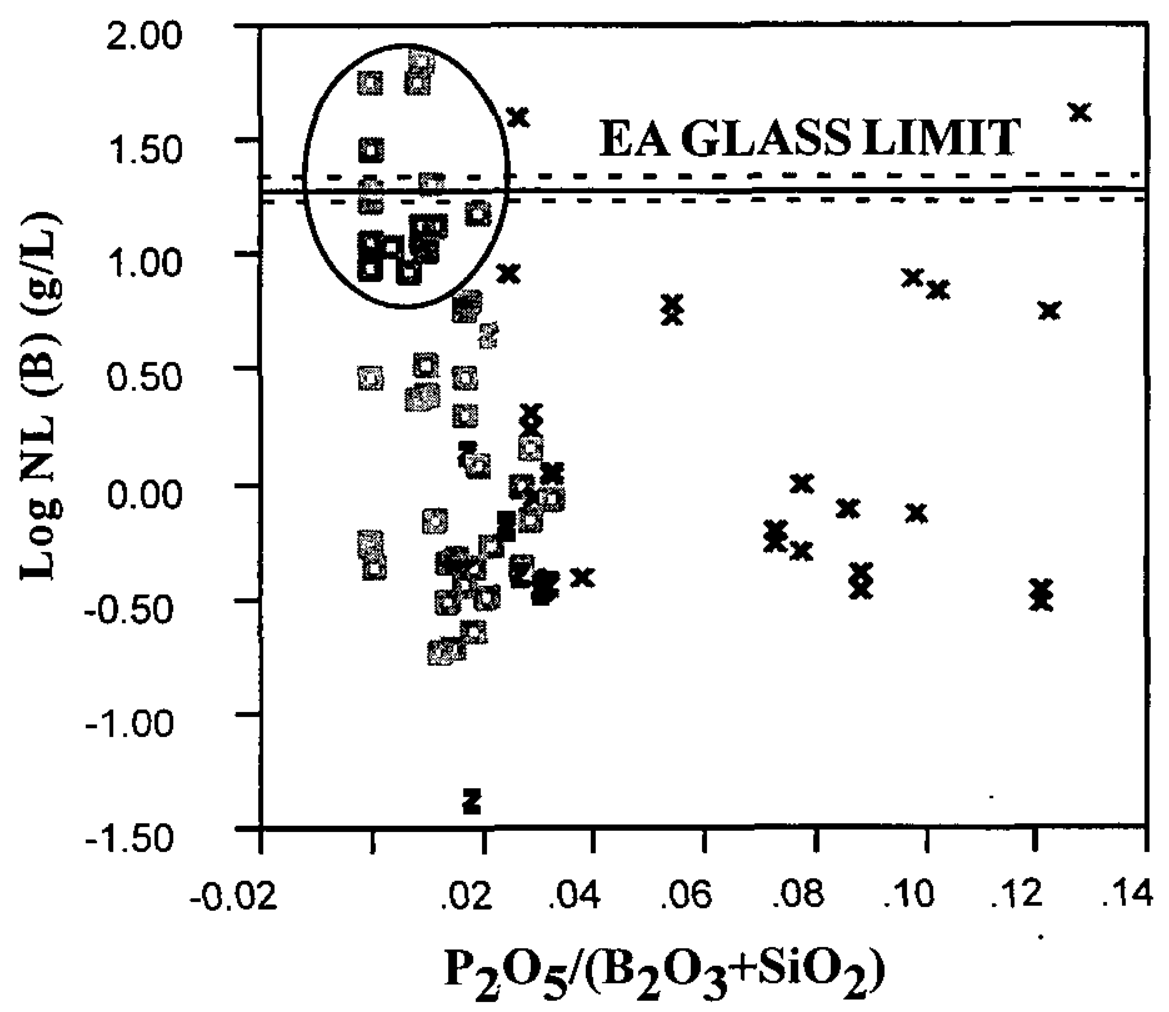

Figure 11a.The durability of the $\mathrm{P}_{2} \mathrm{O}_{5}$ containing borosilicate glasses as a function of the $\mathrm{P}_{2} \mathrm{O}_{5} /\left(\mathrm{SiO}_{2}+\mathrm{B}_{2} \mathrm{O}_{3}\right)$ molar ratio. Borosilicate waste glasses that do not undergo CPS are shown as squares. Glasses that undergo CPS are indicated with darker text ( $x$ and $z$ ). Only a few CPS glasses and many APS glasses are less durable than the Environmental Assessment (EA) glass. The horizontal lines are the EA glass mean durability response ${ }^{14}$ and the upper and lower $2 \sigma$ durability limits. ${ }^{15}$ 


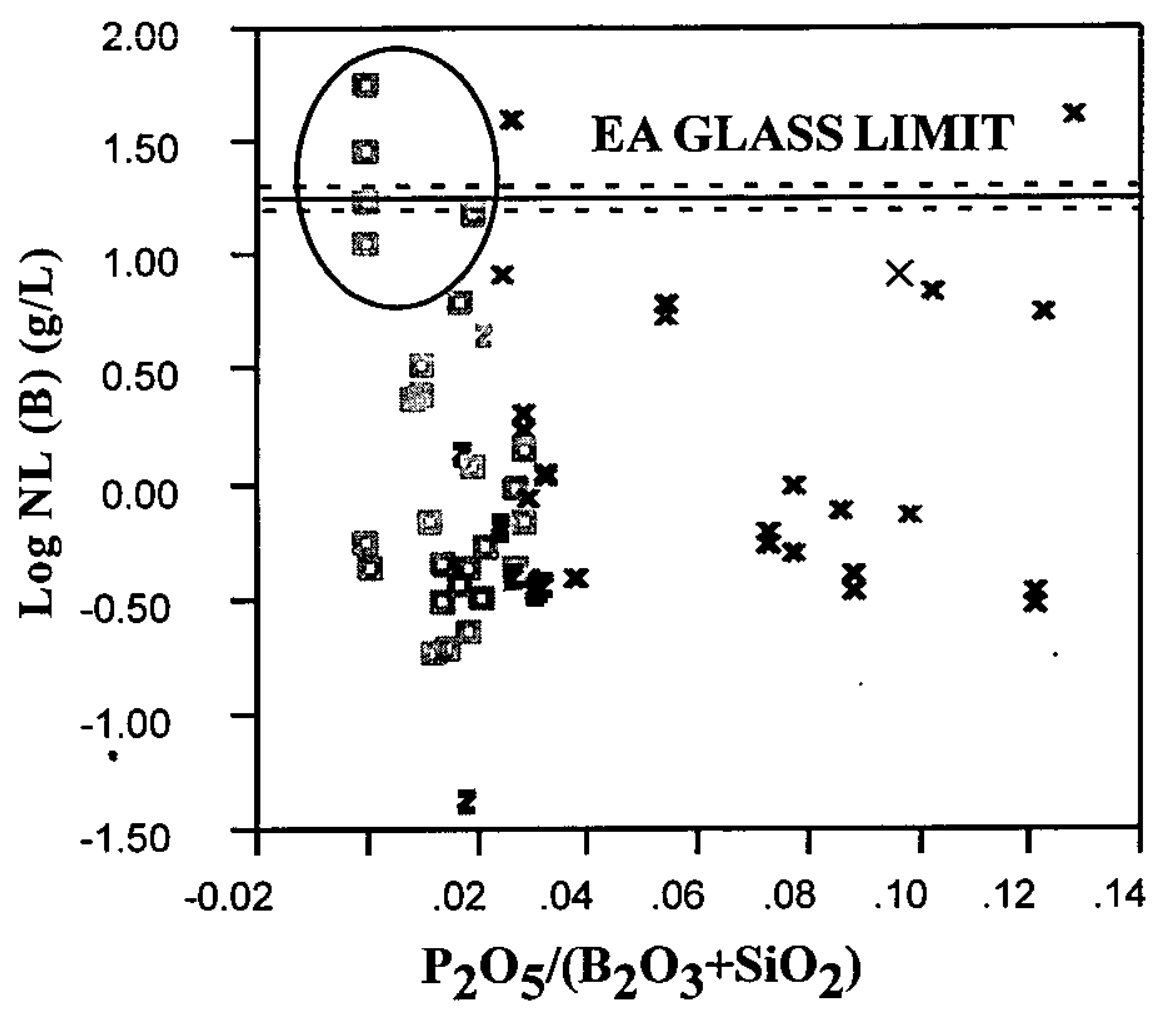

Figure $11 \mathrm{~b}$. Borosilicate glasses with $\geq 14 \mathrm{wt} \% \mathrm{~B}_{2} \mathrm{O}_{3}$ have been removed ( 20 glasses in all) since the data of Tovena et.al. ${ }^{4}$ indicates that glasses with high $\mathrm{B}_{2} \mathrm{O}_{3}$ content undergo APS. This leaves only a few glasses within the ellipse that are less durable than the EA glass limit. 


\subsection{PREDICTING GLASS DURABILITY FOR HIGH $\mathrm{P}_{2} \mathrm{O}_{5}$ CONTAINING GLASSES WITH CRYSTALLINE PHASE SEPARATION (CPS)}

The data of Tovena, et. al. ${ }^{4}$ indicates that borosilicate glasses undergo APS when the $\mathrm{B}_{2} \mathrm{O}_{3}$ content of a glass is $\leq 2 \mathrm{wt} \%$ or between 10 and $15 \mathrm{wt} \%$. If the glasses with $\leq 2$ wt $\% \mathrm{~B}_{2} \mathrm{O}_{3}$ and $\geq 14 \mathrm{wt} \% \mathrm{~B}_{2} \mathrm{O}_{3}$ glasses are removed from the 82 glass database, then most of the poorly durable glasses within the ellipse shown on Figure 11a are removed (compare Figure 11a to $11 \mathrm{~b}$ ). Sixty-two glasses with $\mathrm{B}_{2} \mathrm{O}_{3}$ ranges between 4.15 and 13.7 remain in Figure $11 \mathrm{~b}$, e.g. glasses with $\mathrm{SiO}_{2} / \mathrm{B}_{2} \mathrm{O}_{3}$ ratio (by wt\%) of $<3.81$ are excluded (see Tables I and V). This removes most of the poorly durable glasses which are suspected to have undergone APS. These glasses all have $\leq 2.87 \mathrm{wt} \% \mathrm{P}_{2} \mathrm{O}_{5}$. The glasses remaining in the poorly durable glass ellipse and above the EA glass limit shown in Figure $11 \mathrm{~b}$ are poorly durable homogeneous glasses.

It is well known that glass compositions with less total alkali undergo less ion exchange and are relatively more durable. Glasses with high excess alkali create excess strong base in the leachates during static durability testing. Leachates with less alkali remain buffered in terms of leachate strong bases [SB] and weak acids [WA]. Plotting the [SB][WA] equilibria values from Table 2 for the remaining 62 glasses in Figure 12 demonstrates that glasses with high alkali are the glasses that have high leachate $\mathrm{pH}$ values where excess strong base [SB] is the dominant leachate species. Figure 12 demonstrates that the poor durability of the glasses in the ellipse is due to the excess [SB] and not the CPS.

For the DWPF, glasses with $\leq 19.3 \mathrm{wt} \%$ total alkali $\left(\mathrm{Na}_{2} \mathrm{O}+\mathrm{Li}_{2} \mathrm{O}+\mathrm{Cs}_{2} \mathrm{O}+\mathrm{K}_{2} \mathrm{O}\right)$ have been found to remain durable because the leachates are buffered in terms of their [WA][SB] equilibria. Imposing the DWPF total alkali constraint to the remaining 62 glasses in the database demonstrates that the $\mathrm{P}_{2} \mathrm{O}_{5}$ containing borosilicate glasses remaining are very durable. Indeed, the usage of the $19.3 \mathrm{wt} \%$ total alkali DWPF limit appears to be overly conservative for these high $\mathrm{Al}_{2} \mathrm{O}_{3}$ and high $\mathrm{ZrO}_{2}$ containing glasses as only 21 of the original 62 glasses in the database remain (Figure 13a). Optimizing the total alkali constraint for the $\mathrm{P}_{2} \mathrm{O}_{5}$ containing high $\mathrm{Al}_{2} \mathrm{O}_{3}$ and high $\mathrm{ZrO}_{2}$ borosilicate glasses suggests a limit of $\sim 22 \mathrm{wt} \%$ total alkali. This defines a durable glass population of 35 of the original 62 glasses (Figure 13b): some glasses are homogeneous (squares) and some glasses have undergone CPS (symbols $\mathrm{x}$ and $\mathrm{z}$ ). 


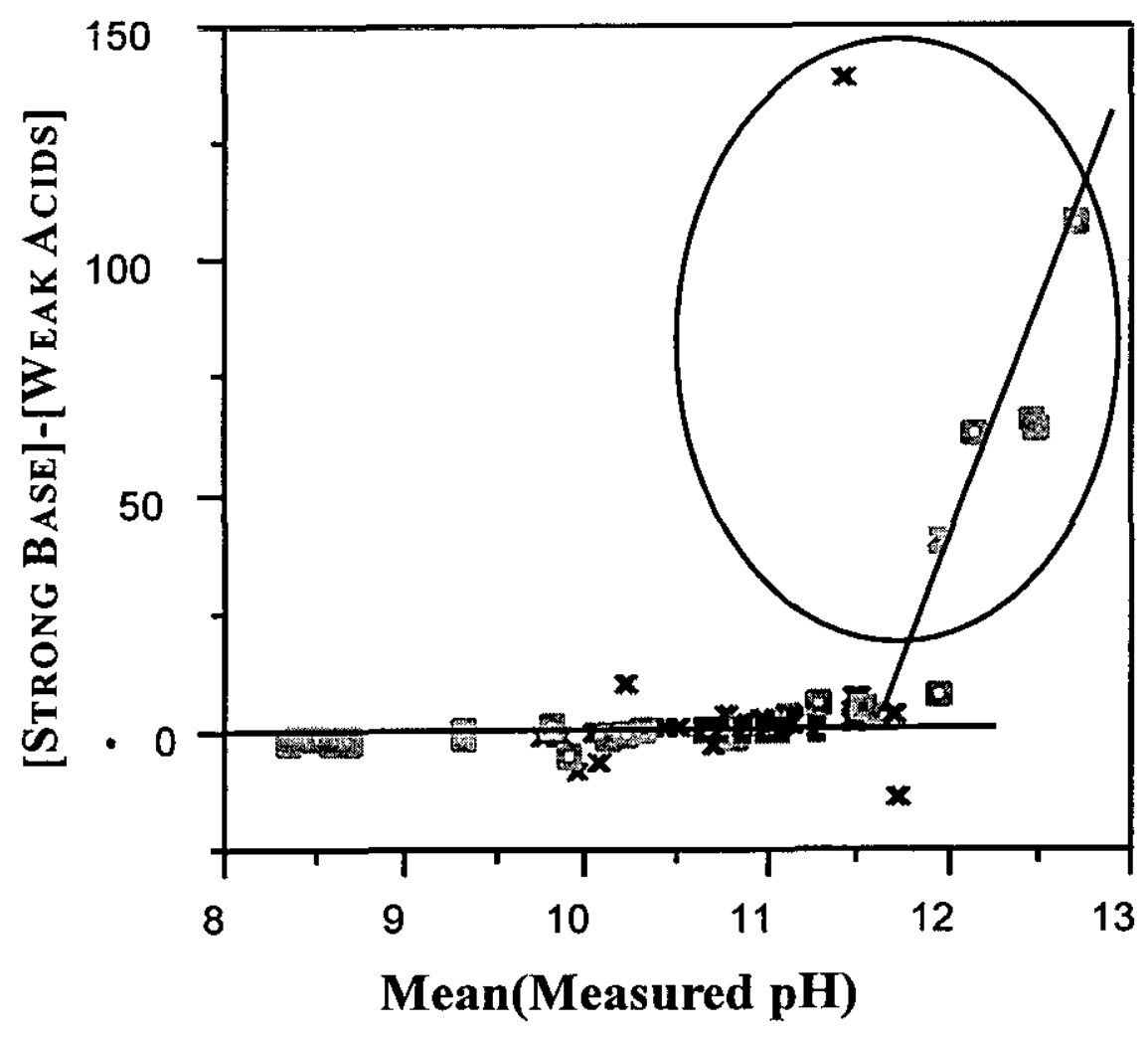

Figure 12. Glass durability is a function of the strong base and weak acid equilibria in the leachate and not a function of the CPS. Glasses indicated with squares are homogeneous. Glasses indicated with the symbols $\mathrm{x}$ and $\mathrm{z}$ have undergone CPS. Glasses in the ellipse have excess strong base, [SB], and are poorly durable. 
WSRC-TR-2000-00339

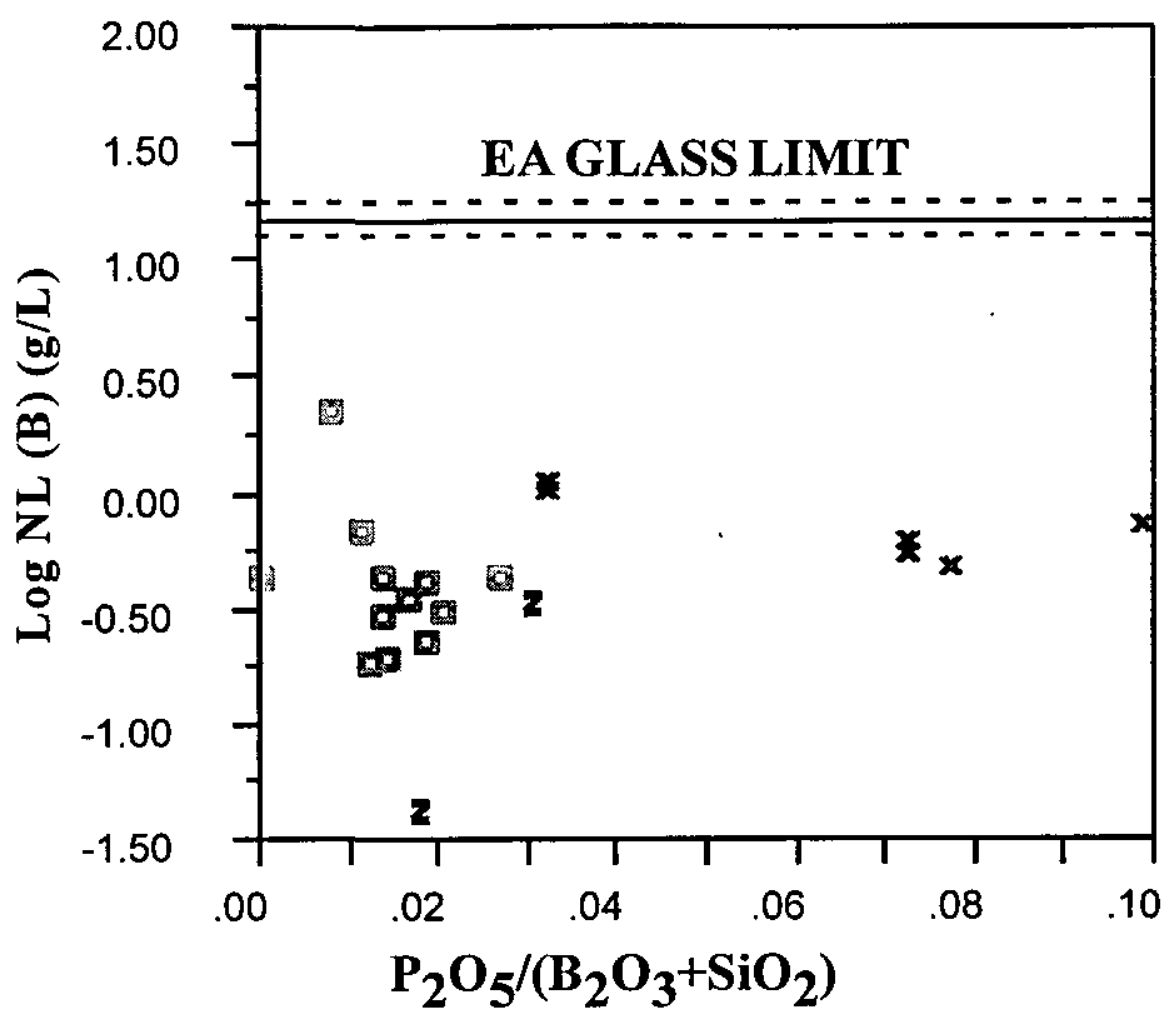

Figure 13a.Durable glass population when $\Sigma$ alkali oxides is limited to $19.3 \mathrm{wt} \%$.

Squares are homogeneous glasses while the symbols $\mathrm{x}$ and $\mathrm{z}$ indicate glasses that have undergone CPS. 


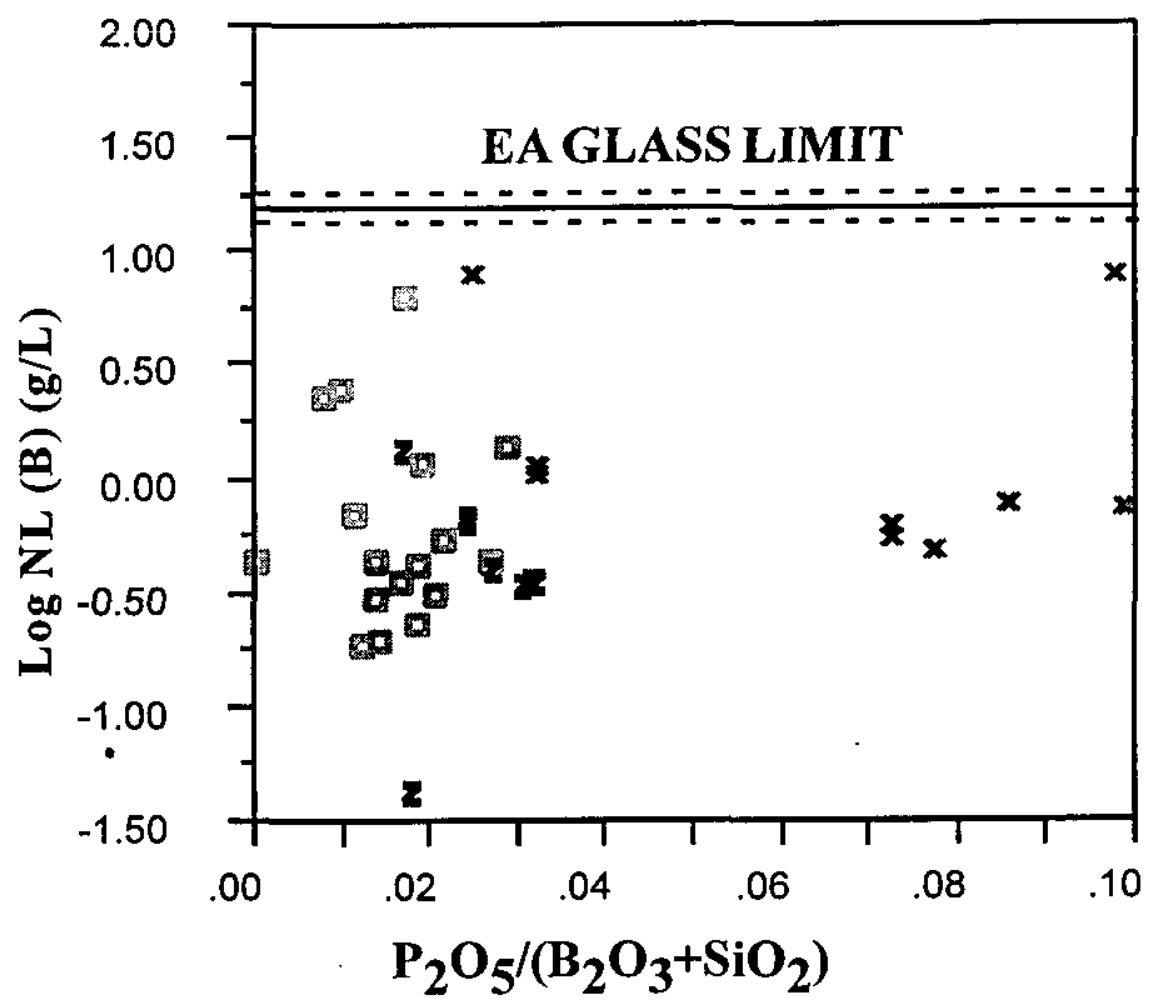

Figure 13b.Durable glass population when $\Sigma$ alkali oxides is limited to $22 \mathrm{wt} \%$. Squares are homogeneous glasses while the symbols $\mathrm{x}$ and $\mathrm{z}$ indicate glasses that have undergone CPS. 
The glass durability is a complex function of the $\mathrm{B}_{2} \mathrm{O}_{3}$ and total alkali in the glass. If the $\mathrm{B}_{2} \mathrm{O}_{3}$ limit causing APS is lowered from $14 \mathrm{wt} \%$ to $10 \mathrm{wt} \%$ then the total alkali limit can be raised from $22 \mathrm{wt} \%$ to $24 \mathrm{wt} \%$ and the same glass durability retained. In order to represent this graphically, the $\log$ normalized $B$ release from Table II for the 82 glasses shown in Figure 11a were contoured (Figure 14a) on a plot of total alkali vs. $\mathrm{B}_{2} \mathrm{O}_{3}$ in wt\%. Most of the durable glasses fall in the lower left quadrant defined by the horizontal line at $24 \mathrm{wt} \%$ total alkali $\left(\mathrm{R}_{2} \mathrm{O}\right)$ and the vertical line $10 \mathrm{wt} \% \mathrm{~B}_{2} \mathrm{O}_{3}$.

Values of $\log \mathrm{NL}(\mathrm{B})$ that are $\geq 1.0 \mathrm{~g} / \mathrm{L}$ are shown in Figure $14 \mathrm{~b}$. These values of $\log$ NL(B) are at or above the EA glass limit of $1.22 \mathrm{~g} / \mathrm{L}$ or its lower $95 \%$ confidence band. The glasses contain $\mathrm{R}_{2} \mathrm{O} \geq 24 \mathrm{wt} \% \mathrm{R}_{2} \mathrm{O}$ and $\geq 10 \mathrm{wt} \% \mathrm{~B}_{2} \mathrm{O}_{3}$. Note that there are glasses shown in Figure $14 \mathrm{~b}$ that have acceptable durabilities $(\leq 1.0 \mathrm{~g} / \mathrm{L} \log$ normalized $\mathrm{B})$ at $>10 \mathrm{wt} \% \mathrm{~B}_{2} \mathrm{O}_{3}$. However, if the $\mathrm{B}_{2} \mathrm{O}_{3}$ value is raised to $14 \mathrm{wt} \% \mathrm{~B}_{2} \mathrm{O}_{3}$, then the $\mathrm{R}_{2} \mathrm{O}$ level has to be lowered to $22 \mathrm{wt} \% \mathrm{R}_{2} \mathrm{O}$ to avoid the poorly durable glasses releasing 1.19 and $1.06 \mathrm{~g} / \mathrm{L} \log$ normalized $\mathrm{B}$ shown in Figure $14 \mathrm{~b}$.

The resulting durable composition domain for $\mathrm{P}_{2} \mathrm{O}_{5}$ containing borosilicate glasses, whether they are homogeneous or have undergone CPS, is given in Table VI as an indication of new composition regions to be assessed for INEEL waste glasses. In order to avoid glasses with excess [SB] that have poor glass durability the $\mathrm{R}_{2} \mathrm{O}$ limit recommended in this paper is $\leq 22 \mathrm{wt} \%$ with a $\mathrm{B}_{2} \mathrm{O}_{3}$ limit of $\leq 14 \mathrm{wt} \%$.

Table VI. Compositional Region Defined for Durable High $\mathrm{P}_{2} \mathrm{O}_{5}$ Containing Borosilicate Glasses Including Homogeneous and CPS Glasses

\begin{tabular}{|l|l|l|}
\hline $\begin{array}{l}\text { Glass Compositional } \\
\text { Component(s) }\end{array}$ & Ranges (wt \%) & Reason \\
\hline $\mathrm{B}_{2} \mathrm{O}_{3}$ & $\geq 2 \mathrm{wt} \%$ and $\leq 14 \mathrm{wt} \%$ & Avoid APS \\
\hline $\begin{array}{l}\mathrm{Na}_{2} \mathrm{O}+\mathrm{Li}_{2} \mathrm{O}+\mathrm{Cs}_{2} \mathrm{O}+ \\
\mathrm{P}_{2} \mathrm{O}_{5}\end{array}$ & $\begin{array}{l}\text { Avoid poorly durable } \\
\text { glasses that form excess } \\
\text { [SB] during durability test }\end{array}$ \\
\hline $\mathrm{Al}_{2} \mathrm{O}_{3}$ & $0.06-13.25$ & Range tested in this study \\
\hline $\mathrm{ZrO}_{2}$ & $0-25$ & Range tested in this study \\
\hline $\mathrm{Al}_{2} \mathrm{O}_{3}+\mathrm{ZrO}_{2}$ & $0-15$ & Range tested in this study \\
\hline & $\geq 9$ & $\begin{array}{l}\text { To improve durability and } \\
\text { stabilize glass against APS } \\
\text { in presence of excess alkali } \\
\text { and } \mathrm{B}_{2} \mathrm{O}_{3}\end{array}$ \\
\hline
\end{tabular}




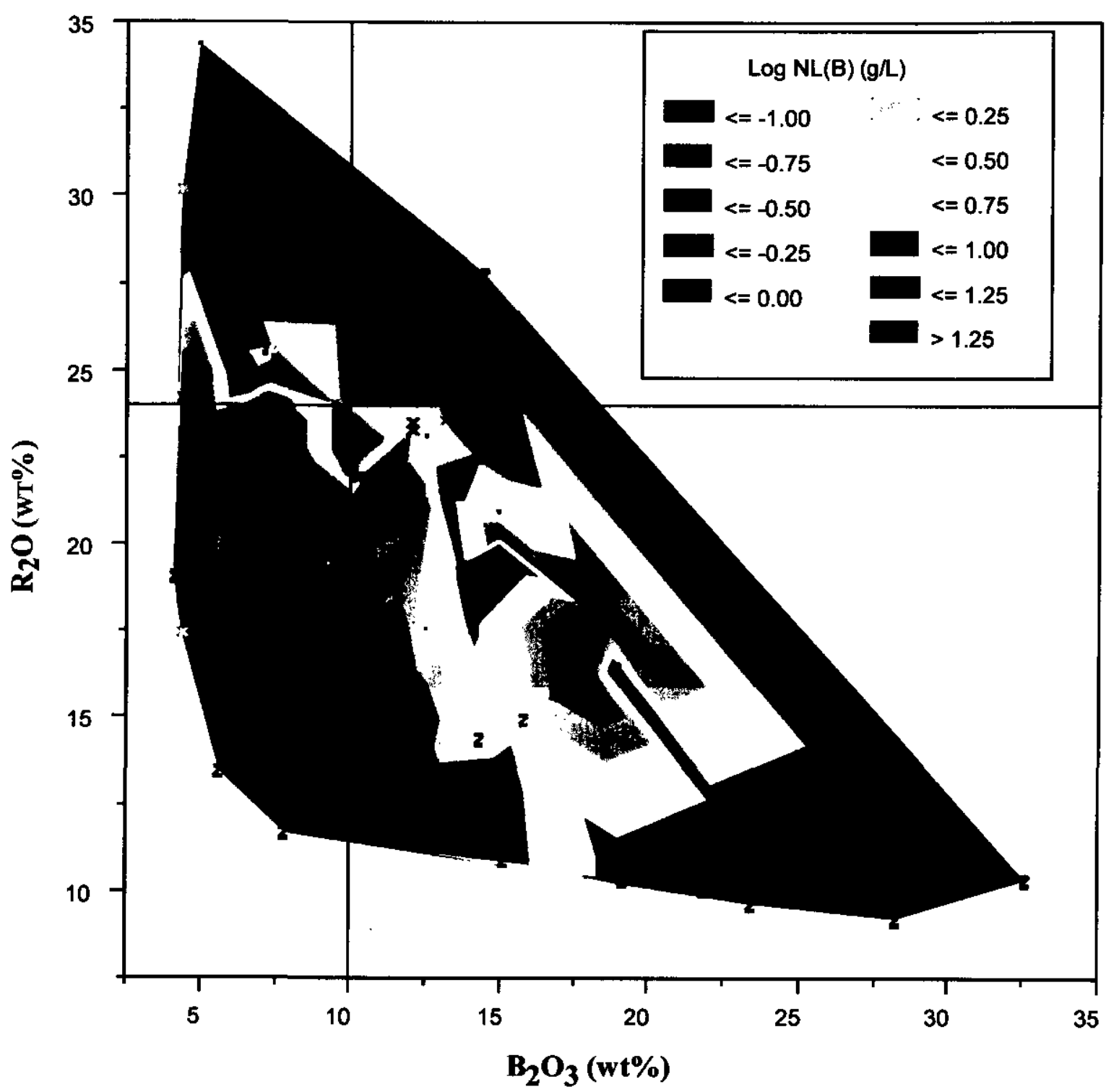

Figure 14a.Contour plot of the PCT leachate response, $\log$ normalized $\mathrm{B}$ in $\mathrm{g} / \mathrm{L}$, demonstrating that values of $\leq 1.0 \mathrm{~g} / \mathrm{L}$ are released from $\mathrm{P}_{2} \mathrm{O}_{5}$ containing glasses with $<10$ wt $\% \mathrm{~B}_{2} \mathrm{O}_{3}$ and $\leq 24 \mathrm{wt} \% \mathrm{R}_{2} \mathrm{O}$ where $\mathrm{R}_{2} \mathrm{O}=\Sigma \mathrm{Na}_{2} \mathrm{O}+\mathrm{Cs}_{2} \mathrm{O}+$ $\mathrm{K}_{2} \mathrm{O}$. 


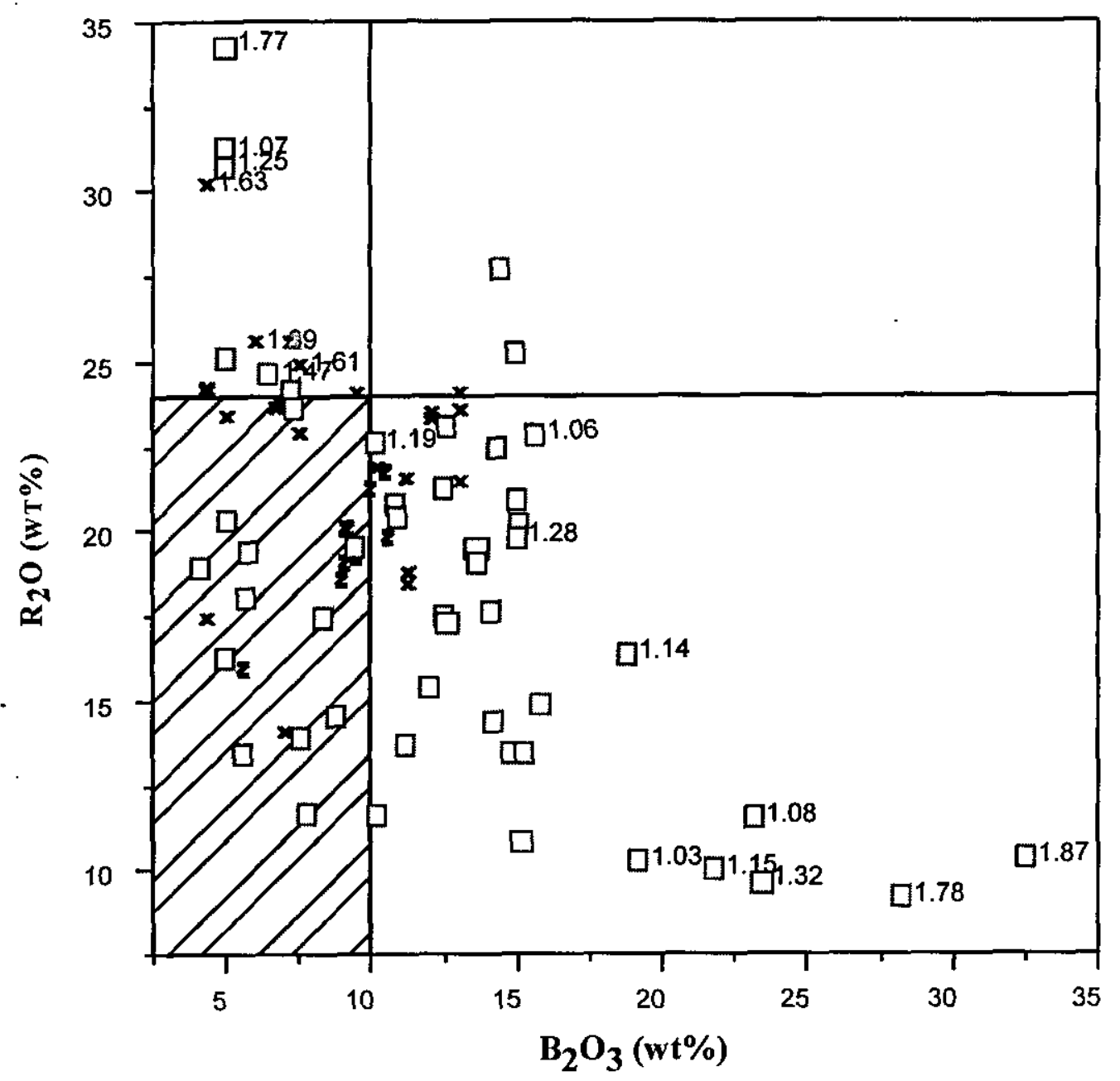

Figure 14b.Individual PCT leachate responses, $\log$ normalized B in $\mathrm{g} / \mathrm{L}$, demonstrating that values of $\leq 1.0 \mathrm{~g} / \mathrm{L}$ are released from $\mathrm{P}_{2} \mathrm{O}_{5}$ containing glasses with $<10 \mathrm{wt} \%$ $\mathrm{B}_{2} \mathrm{O}_{3}$ and $\leq 24 \mathrm{wt} \% \mathrm{R}_{2} \mathrm{O}$ where $\mathrm{R}_{2} \mathrm{O}=\Sigma \mathrm{Na}_{2} \mathrm{O}+\mathrm{Cs}_{2} \mathrm{O}+\mathrm{K}_{2} \mathrm{O}$. Note that if the $\mathrm{B} 2 \mathrm{O} 3$ limit is raised to $14 \mathrm{wt} \%$ that the $\mathrm{R} 2 \mathrm{O}$ must be lowered to $22 \mathrm{wt} \%$ to avoid the nondurable glasses releasing 1.19 and $1.06 \log$ normalized B, respectivley. Glasses indicated with a square are homogeneous glasses while glasses indicted with a $\mathrm{z}$ or $\mathrm{x}$ have undergone crystalline phase separation. The shaded lower left quadrant defines the compositional region of durable glasses regardless of whether the glass is homogeneous or has undergone CPS. 


\subsection{CONCLUSIONS}

- Two types of phase separation occur in the borosilicate waste glasses studied: amorphous phase separation (APS) is predicted in high $\mathrm{B}_{2} \mathrm{O}_{3}$ containing borosilicate waste glasses with $\leq 2.6 \mathrm{wt} \% \mathrm{P}_{2} \mathrm{O}_{5}$ while crystalline phase separation (CPS) occurs in borosilicate waste glasses with $>2.6 \mathrm{wt} \% \mathrm{P}_{2} \mathrm{O}_{5}$.

- In $\mathrm{P}_{2} \mathrm{O}_{5}$ the borosilicate waste glasses studied, the major glass constituents (alkalis and boria) control the glass durability and not the CPS.

- Based on the normalized B and Na release, APS appears to be detrimental to waste glass durability (as determined previously) while CPS does not.

- Due to the crystalline nature of the $\mathrm{P}_{2} \mathrm{O}_{5}$ phase separation and the fact that the CPS phases have little impact on glass durability, it is recommended that CPS should be treated as a crystallization effect rather than as a phase separation effect for waste acceptance. It remains to be demonstrated that the radionuclides do not preferentially partition into the CPS phase and leach at a greater rate than the B or Na. It also must be shown that the formation of the phosphate rich phase does not adversely effect the processability of the glass.

- $\mathrm{U}_{3} \mathrm{O}_{8}$, the only radionuclide studied, did not partition into any CPS phases.

- Alumina was not found to stabilize the glass against CPS in the manner in which it stabilizes borosilicate glasses without $\mathrm{P}_{2} \mathrm{O}_{5}$ from APS.

- Alumina and zirconia were found to stabilize the CPS glass matrix against APS and stabilize the homogeneous glasses against APS: all INEEL glasses with a combined concentration of $\mathrm{Al}_{2} \mathrm{O}_{3}$ and $\mathrm{ZrO}_{2} \geq 9 \mathrm{wt} \%$ were found to have adequate durability.

\subsection{ACKNOWLEDGEMENT}

This paper was prepared in connection with work done under Contract No. DE-AC0996SR 18500 with the U.S. Department of Energy. This work was supported by the Tank Waste Focus Area (TFA) under Technical Task Plan (TTP) SR1-6-WT-31. The M-Area Glass Vitrification Studies were supported by the Mixed Waste Focus Area (EM-50) under TTP 


\subsection{REFERENCES}

1 A.D. Cozzi and C.M. Jantzen, “ Glass Durability Along the Compositional Continuum Between a Phase Separated Frit and a Homogeneous Glass," Ceramic Transactions, V. 107, G. T. Chandler (Eds.), 301-308 (2000).

2 C.M. Jantzen, J.B. Pickett, K.G. Brown, T.B. Edwards, U.S. Patent \#846,278, "Method of Determining Glass Durability (THERMO)," (1998).

3 P.R. Hrma, D.K. Peeler, et al., "Property/Composition Relationships for Hanford High-Level Waste Glass Melting at $1150^{\circ} \mathrm{C}$," U.S. DOE Report PNL-10359, Vols. 1 and 2, Pacific Northwest National Laboratory, Richland, WA (December, 1994).

4 I. Tovena, T. Advocat, D. Ghaleb, E. Vernaz and F. Larche, "Thermodynamic and Structural Models Compared with the Initial Dissolution Rates of SON Glass Samples," Sci. Basis for Nucl. Waste Mgt., XVII, A. Barkatt and R.A. Van Konynenburg (Eds.), Mat. Res. Soc., Pittsburgh, PA, 595-602 (1994).

5 C.M. Jantzen and K.G. Brown, "Impact of Phase Separation on Waste Glass Durability," Ceramic Transactions, V. 107, G. T. Chandler (Eds.), 289-300 (2000).

6 B.A. Staples, D.K. Peeler, G.F. Piepel, J.D. Vienna, B.A. Scholes, and C.A. Musick, "The Preparation and Characterization of INTEC HAW Phase 1 Composition Variation Study Glasses," U.S. DOE Report INEEL/EXT-98-00970 (September, 1998).

7 W. Vogel, “Chemistry of Glass,” Am. Ceram. Soc., Columbus, OH, pp.111-113 (1985).

8 M. Tomazawa, "Phase Separation in Glass," in, Treatise on Mat. Sci. and Technology, Vol. 17, Eds. M. Tomazawa and R.H. Doremus, pp. 71-113, Academic Press, NY (1979).

9 C.M. Jantzen, "Phosphate Additions to Borosilicate Waste Glass Cause Phase Separation," U.S. DOE Report DPST-86-389, E.I. duPont deNemours \& Co., Aiken, SC (April 15, 1986). 
10 M.H. Langowski, H. Li, P. Hrma, M.J. Schweiger and D.E. Smith, "The Effect of Phosphate on Crystallization, Viscosity, and Chemical Durability of Simulated Hanford Site High-Level Radioactive Waste Glasses," Ceramic Transactions, V. 72, V. Jain and D.K. Peeler (Eds.) Amer. Ceram. Soc., Westerville, OH, pp. 291198 (1996).

11 A.D. Cozzi, "Technical Status Report on the Effect of Phosphate and Aluminum on the Development of Amorphous Phase Separation in Sodium Borosilicate Glasses," U.S. DOE Report WSRC-TR-98-00327, Rev. 1, Westinghouse Savannah River Co., Aiken, SC (Nov. 30, 1998).

12 S.N. Crichton, T.J. Barbieri, and M. Tomozawa, "Solubility Limits for Troublesome Components in a Simulated Low Level Nuclear Waste Glass," Environmental Issues and Waste Management Technologies in the Ceramic and Nuclear Industries, V. Jain and R. Palmer (Eds)., Ceramic Transactions, V. 61, Amer. Ceram. Soc., Westerville, OH, 283-290 (1995).

13 M.B. Volf, “Chemical Approach to Glass,” Glass Science and Technology, V. 7, Elsevier Science Publishing Co., Inc, New York, 594 pp (1984).

14 C.M. Jantzen, N.E. Bibler, and D.C. Beam, "Characterization of the Defense Waste Processing Facility (DWPF) Environmental Assessment (EA) Glass Standard Reference Material," U.S. DOE Report WSRC-TR-92-346, Westinghouse Savannah River Co., Aiken, SC (September, 1992).

15 Office of Environmental Restoration and Waste Management, U.S. DOE, "Waste Acceptance Product Specifications for Vitrified High-Level Waste Forms," U.S. DOE Report EM-WAPS, Rev. 2 (December, 1996). 
Distribution - Tank Focus Area:

DOE-SR

Thomas S. Gutmann

Sharon J. Robinson

George Mishra

Westinghouse Savannah River Company

Jerome P. Morin

David W. Freeman

Heatherley H. Dukes

John C. Musall

Document Control (4 copies)

Savannah River Technology Center

Lucien M. Papuchado

E. William Holtzsheiter, TIM

Renee H. Spires

David A. Crowley

John R. Harbour

Sharon L. Marra

Rebecca L. Schulz

Thomas B. Edwards

Alex D. Cozzi

David K. Peeler

Terry K. Snyder

Gayle S. Baumgarner

Troy Lorier

Johnetta C. George

Pacific Northwest National Laboratory

Robert W. Allen

Ronda A. Biaggi

Thomas M. Brouns

Betty A. Carteret

Roger L. Gilchrist

Stephen N. Schlahta

Joseph H. Westsik

L.M. Peurrung

Terri L. Stewart

John D. Vienna

Pavel Hrma

Idaho National Engineering and Environmental Laboratory

Jim Rindfleisch

Rich Tillotson

Arlin Olson

Chris A. Musick

Brad A. Scholes 
Westinghouse Savannah River Company Document Approval Sheet

Title

Crystalline Phase Separation in Phosphate Containing Waste Glasses: Relevance to INEEL HAW (U)

Primary Author/Contact (Must be WSRC)

Carol M. Jantzen

Organization Code

L3100A

Other Authors

Kevin G. Brown (WSRC), John B. Pickett (WSRC) and Gary L. Ritzhaupt (Oral Roberts Univ)

Phone No
$5-2374$

Location 5-2374

Organization (No Abbreviations)

Savannah River Technology Center/Immobilization Technology Section

Document No.

WSRC-TR-2000-00339, Revision 0

Key Words (ist 3)

high activity waste, vitrification

Position User ID

Advisory Sci.

T6289 has an invention disclosure, patent application or copyright application $\square$ Yes $\otimes$ No lf yes, date submitted
been submitted related to this information?

Disclosure No. (If Known)

If no, do you intend to submit one?

$\square$ Yes $\otimes$ No

Title

Information Product Description

$\square$ Technical Report

$\square$ Semiannual $\square$ Annual $\otimes$ Final $\square$ Topical $\square$ Other

$\square$ Administrative Report

$\square$ Semiannual $\square$ Annual $\square$ Final $\square$ Topical $\square$ Other

$\square$ Videotape/Multimedia

$\square$ External Web Page URL

[] Brochure/Booklet

$\square$ Procedure/User Guide

$\square$ Drawing

$\square$ Software Package

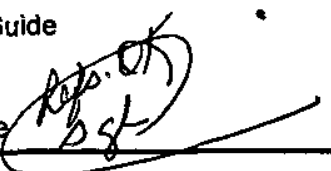

References $\otimes$ in Public Literature

$\square$ Routing Concurrently

If yes, projected date.

$\square$ Journal Article Journal Name

Book/Book Chapter Book Name

$\square$ Conference Submission*

$\square$ Abstract $\square$ Conf. Paper $\square$ Conf. Proceeding

$\square$ Slides/poster/display

$\square$ Other

Approval Requested by (date) 9/30/2000

"Conference Title

*Conference Location (City, State, Country)

*Conference Dates $\mathrm{m} / \mathrm{d} / \mathrm{y}$ thru $m / d / y$

*Conference Sponsor
Approved for Release

$I$ understand that for the information in this material to be given extemal distribution, approvals by both WSRC and, as appropriate. DOE-SR are required. Distribution (verbally or published) must be in accordance with policies set forth in WSRC management requirements and procedures (MRP 3.25) and in DOE-SR orders, and the content of the external distribution grust be limited to that actually approved.

$$
\text { Cous in fan tien }
$$

$9 / 12 / 00$

\section{Approvals by Author's Organization}

Derivative Classifier.

Soroser

Intended Distifipution

\begin{tabular}{|l|l}
\hline Classification & $\begin{array}{l}\text { Topic } \\
\text { Waste Management }\end{array}$ \\
\hline
\end{tabular}

$\begin{array}{ll}\bigotimes \text { Unlimited (release to public) } & \square \text { Site Use Only } \\ \square, \text { Limited (see explanation) } & \square \text { Other DOE facility(ies) only }\end{array}$

$\square$ Corporate/University Partner

$\square$ Other

T understand and have considered whether any potential intellectual property rights (patents, copyrights, etc., in accordance with MP 1.09 and MRP 1.07) or

any contractural barriers (CRADAs. Work for Others, etc.) may be involved before authorizing that this document be proposed for public release. If any

concerns were identifled, these have been discussed and resolved with General fainset.

Manager's Name (Print)

\begin{tabular}{|lllll|}
\hline Classification (Check One for Each) & & \\
Overall & $\square$ s & $\square$ c & $\square$ UCNI & $\square 0$ \\
Abstract & $\square$ s & $\square$ c & $\square$ UCNI & $\square$ u \\
Title & $\square$ s & $\square \mathrm{c}$ & $\square$ UCNI & $\square 0$ \\
\hline
\end{tabular}

WSRC Classification Officer's Name (Print)

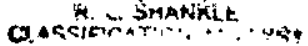

Classificatiog Guide Topics

OPSEC 4 S

wSR glassificafign Officer's Signature

Date

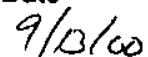

\section{Export Control Related

$$
\square \text { Yes } 4 \text { No }
$$

Export Control Reviewer's Name (Prigt) L. Swankct

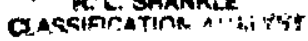

ExporthCogtyolfRevigwer's Signature

OSTI Subj. Category No.

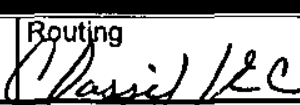

Editor/lilustrator/On-line Support

MSD Project No.

$5 C-12$

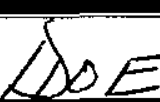

NOTE OSR 17-8 must be completed in additigh to this form when submitting information for review and approval. 


$$
\text { DoE Contact: }
$$

$$
\text { Bill Brasel }
$$

Ms. W. F. Perrin, Technical Information Officer

U. S. Department of Energy - Savannah River Operations Office

Dear Ms. Perrin:

\section{REQUEST FOR APPROVAL TO RELEASE SCIENTIFIC/TECHNICAL INFORMATION}

The attached document is submitted for classification and technical approvals for the purpose of external release. Please complete Part II of this letter and return the letter to the undersigned by $9 / 29 / 2000$. The document has been reviewed for classification and export control by a WSRC Classification staff member and has been determined to be Unclassified.
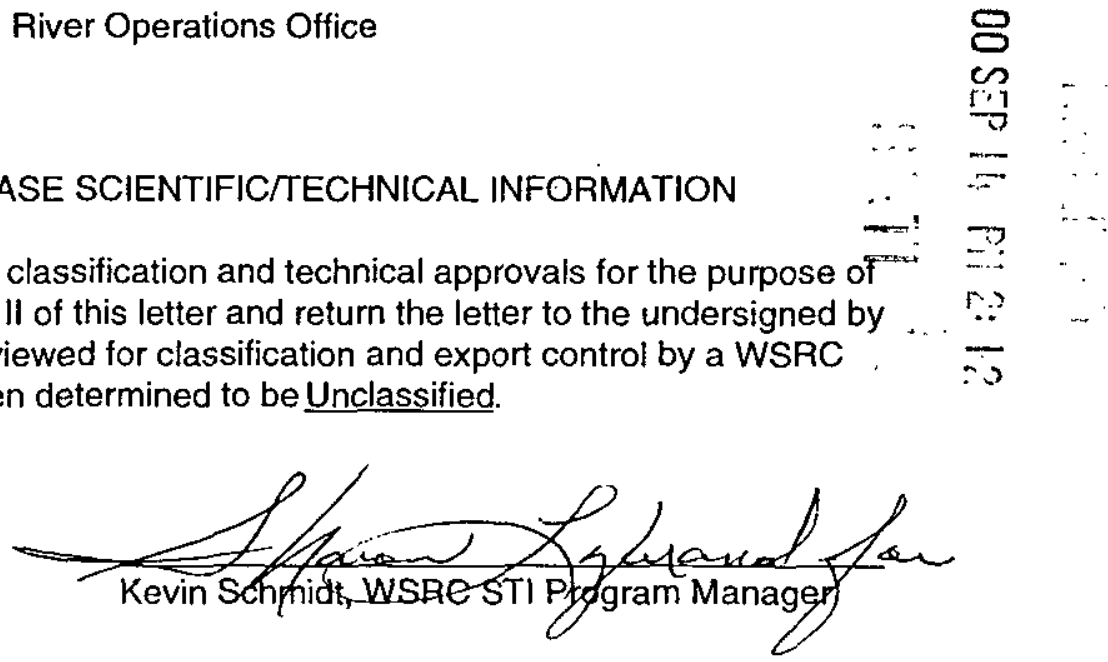

\section{DETAILS OF REQUEST FOR RELEASE}

Document Number: WSRC-TR-2000-00339,

Author's Name: C. M. Jantzen

Location: 773-A

Phone 5-2374

Department: SRTC/Immobilization Technology Section

Document Title: Crystalline Phase Separation in Phosphate Containing Waste Glasses: Relevance to INEEL HAW

Presentation/Publication:

Meeting/Journal:

Location:

Meeting Date:

$$
\text { DSTI Pepartabe }
$$

\section{DOE-SR ACTION}

Date Received by TIO

[X] Approved for Release Not Approved

$\square$ Approved Upon Completion of Changes Revise and Resubmit to DOE-SR

Approved with Remarks

Remarks:
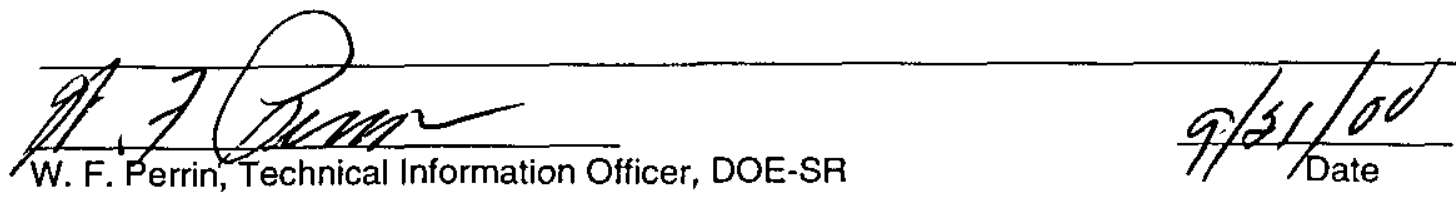
RECORD STATUS (select one):

X...New ......Revised Data -...Revised STI Product

Part I: STI PRODUCT DESCRIPTION

A. STI PRODUCT TYPE (select one)

X.. 1. Technical Report
a. Type:

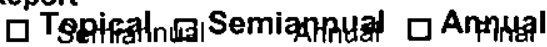
\ Finfópical
Other (specify)

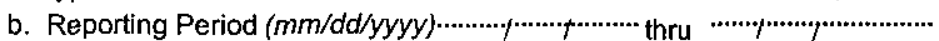

.... 2. Conference
a. Product Type: -..... Conference Proceedings ....
Conference Paper or Other (abstracts, excerpts, etc.)
b. Conference Information (title, location, dates)

.... 3. Software Manual ( The actual software package should be made available simultaneously. Follow instructions provided with ESTSC F 1 and ESTSC F 2.)

… 4. Journal Article
a. Type: $\quad \underline{X}$ Announcement citation only
_. Preprint
Postprint
b. Journal Name
c. Volume
d. Issue
e. Serial identifier (e.g., ISSN or CODEN)

..... 5. S\&T Accomplishment Report

…. 6. Book

…. 7. Patent Application
a. Date Filed $(\mathrm{mm} / \mathrm{dd} / \mathrm{yyyy})$
b. Date Priority $(\mathrm{mm} / \mathrm{dd} / \mathrm{y} / \mathrm{y} y \mathrm{y})$
c. Patent Assignee

\section{.... 8. Thesis/Dissertation}

B. STI PRODUCT TITLE Crystalline.Phase.Separation.in.P.hosphate.Containing.Waste.Glasses:.Relaxance.to.INEEL.HAW..
C. AUTHOR(s) E-mail Address(es):
C.. M. Jantzen.

D. STI PRODUCT IDENTIFIER
1. Report Number(s) WSRC-TR-2000.0.0339. Rex $x_{\text {. }} 0$
2. DOE Contract Number(s) DE-AC.09.96\$R1.9500...
3. R\&D Project ID(s)
4. Other Identifying Number(s)

E. ORIGINATING RESEARCH ORGANIZATION Savannah River Site

F. DATE OF PUBLICATION ( $m m / d d / y y y y)$ 9/2.1/20.90..............

G. LANGUAGE (if non-English) English

(Grantees and Awardees: Skip to Description/Abstract section at the end of Part)

H. SPONSORING ORGANIZATION

I. PUBLISHER NAME AND LOCATION (if other than research organization)

Availability (refer requests to [if applicable])

J. SUBJECT CATEGORIES (list primary one first)

12

Keywords high.actixity..waste..xitrification..phase.separation..phosphate.glas.s..waste.glas.

K. DESCRIPTION/ABSTRACT

As part of the Tanks Focus Area's (IFA) effort to increase waste loading for high-level waste vitrification at various

facilities in the Department of Energy (DOE) complex, the occurrence of phase separation in waste glasses spanning

the Savannah RIver Site (STSS) and ddaho Nationaltengineering and Environmental Laboratory (INEEL) composition

ranges have been studied. The type of phase separation that occurs in the phosphate rich borosilicate waste glasses, such as those investigated for INEEL, crystallizes upon cooling. This type of phase separation mechanism is less well studied than amorphous phase separation in phosphate poor borosillcate waste glasses. Therefore, the type of phase separation, extent, and impact of phase separation on glass durability for a series of INEEL-type glasses were examined and the data statistically analyzed in this study. 


\section{ANNOUNCEMENT OF U. S. DEPARTMENT OF ENERGY (DOE)}

SCIENTIFIC AND TECHNICAL INFORMATION (STI)

Part II: STI PRODUCT MEDIAFORMAT and LOCATION/TRANSMISSION

\section{A. MEDIAIFORMAT INFORMATION}

1. Medium of STI product is: $\quad$....... Paper $\quad$...... Electronic document $\quad$...... Computer medium $\quad$...... Audiovisual material

2. Size of STI product

3. File format:

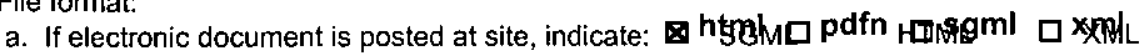
PDF Normal PDF Image
b. If electronic document is transmitted to OSTI, indicate: $\square$ htmbM pdfn BIRdfi $\square$ mskerd
PDF Normal

_ TIFFG4_WP-indicate Version (5.0 or greater)

— MS Word-indicate Version ( 5.0 or greater)

platform/operating system

4. If computer medium or audiovisual material:
a. Quantity/type (specify)
b. Machine compatibility (specify)
c. Sound:
f. Other information about product format a user needs to know:

platform/operating system Postscript

\section{B. LOCATION/TRANSMISSION INFORMATION}

1. STI Product is available at site: Unique URL (of specific STI Product htth:/www.sr.s.gov/general/sci.:tech/fulltext/tr.2000339/tr.2.

2. STI Product is being transmitted to OSTI: Q.0.0.3.3.9.html

a. Electronically via FTP

b.__ Via Mail or shipment (e.g., Federal Express) (Paper products, electronic documents onCD-ROM, diskettes, videocassettes, etc.)

3. Information Product Filename (of transmitted electronic format)

C. ADDITIONAL INFORMATION (concerning media/format or location/transmission; for OSTI internal use only):

(Grantees and Awardees: Skip to Contact section at the end of Part III)

Part III: STI PRODUCT REVIEW? RELEASE INFORMATION

A. ACCESS LIMITATION

X.... 1. Unlimited Announcement (available to U.S. and non-U.S. public)

....... 2. OpenNet (use OpenNet guidance for below):

a. If additional source other than making it available through NTIS:

e. OpenNet Document Type

(1) Accession Number

f. OpenNet Document Keywords

(2) Document Location

b. Field Office Acronym

c. Declassification date ( $\mathrm{mm} / \mathrm{dd} / \mathrm{yyyy})$

d. Declassification Status:

Declassified _ Sanitized _ Never classified

3. U.S. Dissemination Only

….... 4. Copyrighted material; Are there any restrictions based on copyright?

g. OpenNet Addressee yes no. If yes, list the restrictions

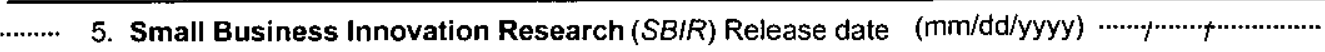

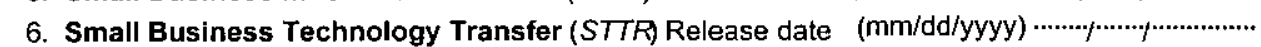

7. Proprietary/Trade Secret

8. Patent Pending

9. Protected data CRADA O Other (specify) Release date $(\mathrm{mm} / \mathrm{dd} / \mathrm{yyyy})$

....... 10. Official Use Only (OUO)

........ 11. Program-Directed Special Handling (specify)

12. Export Control/ITAR/EAR

13. Unclassified Controlled Nuclear Information (UCNI)

14. Classified Classification Level/Category of:
a. This form $\mathbf{U}$.
b. The STI Product
Unclassified.

15. Other information relevant to access (specify; for OSTI internal use only)

B. OTHER (information useful to include in published announcement record which is not suited for any other field on this form)

\section{CONTACT AND RELEASING OFFICIAL.}

1. Contact (if appropriate, the organization or site contact to include in published citations who would receive any external questions about the content of the

STI Product or the research information contained therein ) Kevin Schmidt, Manager STI Program \& Site Support
Name and/or Position

E-mail Phone $(803) 725-2765$

Organization Westtnghouse Savamialtriver Compally

2. Releasing Official $\mathbf{X}$ Kevin Sechfythatall necessary reviews have been completed (e.g. Patent, Copyright, ECI, UCNI etc.)
Released by (name)

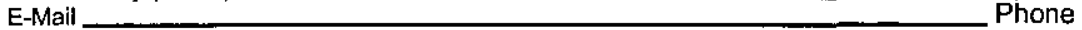

